\title{
ON CORON'S PROBLEM FOR WEAKLY COUPLED ELLIPTIC SYSTEMS
}

\author{
ANGELA PISTOIA AND NICOLA SOAVE
}

AbStract. We consider the following critical weakly coupled elliptic system

$$
\begin{cases}-\Delta u_{i}=\mu_{i}\left|u_{i}\right|^{2^{*}-2} u_{i}+\sum_{j \neq i} \beta_{i j}\left|u_{j}\right|^{\frac{2^{*}}{2}}\left|u_{i}\right|^{\frac{2^{*}-4}{2}} u_{i} & \text { in } \Omega_{\varepsilon} \\ u_{i}=0 & \text { on } \partial \Omega_{\varepsilon},\end{cases}
$$

in a domain $\Omega_{\varepsilon} \subset \mathbb{R}^{N}, N=3,4$, with small shrinking holes as the parameter $\varepsilon \rightarrow 0$. We prove the existence of positive solutions of two different types: either each density concentrates around a different hole, or we have groups of components such that all the components within a single group concentrate around the same point, and different groups concentrate around different points.

\section{INTRODUCTION}

The system of nonlinear elliptic equations

$$
\left\{\begin{array}{l}
-\Delta u+\lambda_{i} u_{i}=\mu_{i}\left|u_{i}\right|^{p-1} u_{i}+\sum_{j \neq i} \beta_{i j}\left|u_{j}\right|^{\frac{p+1}{2}}\left|u_{i}\right|^{\frac{p-3}{2}} u_{i} \quad \text { in } \Omega \quad i=1, \ldots, m \\
u_{i} \in H_{0}^{1}(\Omega)
\end{array}\right.
$$

where $\Omega$ is either a bounded domain or the whole space $\mathbb{R}^{N}$, has been studied intensively in the last decade, in light of its relevance in different physical context: (1.1) appears when looking for solitary wave solutions $\Phi_{i}(t, x)=e^{\iota \lambda_{i} t} u_{i}(x)$ for the coupled Gross-Pitaevskii equation

$$
-\iota \partial_{t} \Phi_{i}=\Delta \Phi_{i}+\mu_{i}\left|\Phi_{i}\right|^{p-1} \Phi_{i}+\sum_{j \neq i} \beta_{i j}\left|\Phi_{i}\right|^{\frac{p-3}{2}}\left|\Phi_{j}\right|^{\frac{p+1}{2}} \Phi_{i}
$$

which is of interest in nonlinear optics and in quantum mechanics, see e.g. [1,30]. In the models, $\left|u_{i}\right|$ represents the amplitude of the $i$-th density, and the real parameters $\mu_{i}$ and $\beta_{i j}$ represent the intra-spaces and inter-species scattering length, describing respectively the interaction between particles of the same component or of different components. In particular, the positive sign of $\mu_{i}$ (and of $\beta_{i j}$ ) stays for attractive interaction, while the negative sign stays for repulsive interaction.

From the mathematical point of view, (1.1) is one of the simplest, yet highly non-trivial, examples of weakly coupled system, i.e. is a system admitting non-trivial solutions $\left(u_{1}, \ldots, u_{m}\right) \not \equiv$ $(0, \ldots, 0)$ with some trivial components $u_{i} \equiv 0$. This feature stimulated a lot of research about existence of fully nontrivial solutions, i.e. solutions with $u_{i} \not \equiv 0$ for every $i$. Nowadays, many results in this direction are available, mainly concerning the cubic problem $p=3$ in dimension $N \leq 3$, i.e. in a Sobolev subcritical regime. A complete review of the result in this framework would be beyond the aim of the present paper, and we refer the interested read to the quite exhaustive introductions

2010 Mathematics Subject Classification. 35J15; 35J20; 35J50.

Key words and phrases. Competitive and Cooperative systems, Blow-up and concentrating solutions, Critical system, Lyapunov-Schmidt reduction.

Acknowlegments. Part of this work was carried out while N. Soave was visiting the University of Rome, "La Sapienza", which he wishes to thank for the hospitality. N. Soave is partially supported through the project ERC Advanced Grant 2013 n. 339958 "Complex Patterns for Strongly Interacting Dynamical Systems - COMPAT". 
in $[28,29]$ and to the references therein. In this paper we focus instead on the much less understood Sobolev critical regime $p=2^{*}-1$ in dimension $N=3$ or $N=4$, where $2^{*}=2 N /(N-2)$ is the critical exponent for the Sobolev embedding $H^{1}\left(\mathbb{R}^{N}\right) \hookrightarrow L^{r}\left(\mathbb{R}^{N}\right)$; moreover, from now on we limit ourselves to the focusing setting $\mu_{i}>0$ for every $i$.

The study of the critical system (1.1) started with the Chen and Zou's paper [9], where the authors focused on (1.1) with 2 components in bounded domains of $\mathbb{R}^{4}$ (thus, with $p=3$ ), and proved existence of least energy positive solutions under suitable assumptions on the parameters $\lambda_{i}, \mu_{i}>0, \beta_{i j}$. In [10], the authors extended their results in higher dimension $N \geq 5$. In both papers it is assumed that $-\nu_{1}(\Omega)<\lambda_{1}, \lambda_{2}<0$ (here $\nu_{1}(\Omega)$ denotes the first eigenvalue of $(-\Delta)$ with homogeneous Dirichlet boundary conditions in $\Omega$ ), and this plays a crucial role: indeed, as remarked by Chen and Zou, system (1.1) with $\Omega$ bounded, $\mu_{i}>0$ and $p=2^{*}-1$ can be considered a critically coupled version of the Brezis-Nirenberg problem

$$
\begin{cases}-\Delta u+\lambda u=|u|^{2^{*}-2} u & \text { in } \Omega \\ u=0 & \text { on } \partial \Omega\end{cases}
$$

and it is well known that in any dimension $N \geq 4$ (1.2) admits a positive solution (for an arbitrary bounded domain) if and only if $-\nu_{1}(\Omega)<\lambda<0$ (see [5] for this result, and the survey [22] for a more extended discussion).

The relation between (1.1) and the Brezis-Nirenberg problem has been recently exploited also in $[8,23]$. In [8], Chen and Lin described the blow-up behaviur of least energy positive solutions as $\lambda_{i} \rightarrow 0$, in case of 2 components system with $\beta_{12}>0$. In [23], the first author and Tavares constructed, under appropriate assumptions on the domain $\Omega \subset \mathbb{R}^{4}$ and on the parameters $\beta_{i j} \in \mathbb{R}$, solutions to (1.1) with all the components $u_{i}$ concentrating around different points $a_{i} \in \Omega$ as $\lambda_{i} \rightarrow 0^{-}$.

We finally refer to [15], where the authors proved existence of infinitely many non-radial solutions for (1.1) in $\mathbb{R}^{3}$, with $\lambda_{i}=0$ and $\beta_{i j}<0$.

In this paper we address (1.1) when $\Omega$ is a bounded domain of $\mathbb{R}^{3}$ or $\mathbb{R}^{4}$ and $\lambda_{i}=0$ for every $i$. If $\Omega$ is star-shaped, the Pohozaev identity for gradient-type systems implies that the problem has no nonnegative solutions (meaning that $u_{i} \geq 0$ for every $i$ ) but the trivial one $\left(u_{1}, \ldots, u_{m}\right)=(0, \ldots, 0)$, but if $\Omega$ has some hole there is hope to find fully nontrivial positive solutions, in the spirit of the celebrated Coron and Bahri-Coron results, which we briefly review: let us consider the critical problem

$$
\left\{\begin{array}{ll}
-\Delta u=|u|^{2^{*}-2} u & \text { in } \Omega \\
u>0 & \text { in } \Omega \\
u=0 & \text { on } \partial \Omega,
\end{array} \text { with } \Omega\right. \text { bounded domain. }
$$

If $\Omega$ is star-shaped, non-trivial solutions do not exist, but the situation drastically changes removing this geometric assumption: indeed, as observed by Kazdan and Warner in [16], (1.3) in an annulus admits a positive solution. The result was then improved by Coron, who showed in [13] that (1.3) has a positive solution as long as $\Omega$ has a small hole. A further improvement was achieved by Bahri and Coron, who proved in [2] that a positive solution does exist provided that $\Omega$ has non-trivial topology. For multiplicity results and sign changing solutions, we refer the interested reader to $[11,12,14,18,21,24]$.

The purpose of this paper is to discuss the extension to (1.1) of the Coron result. While in [13] a variational argument is considered, we adopt here a perturbation approach based on the LyapunovSchmidt finite dimensional reduction, which has been already fruitfully used to deal with scalar 
Coron's type problem in $[11,14,20,21]$. In order to state our main result, we introduce some notation and recall some basic results.

We consider from now on the following general assumptions, which will not be always recalled. Let $\Omega \subset \mathbb{R}^{N}$ with $N=3,4$ be a bounded and sufficiently regular domain, and let $m \in \mathbb{N}$. Let $a_{1}, \ldots, a_{m} \in \Omega$ be $m$ (not necessarily different) points in $\Omega, r_{1}, \ldots, r_{m}>0, \mu_{1}, \ldots, \mu_{m}>0$ and $\beta_{i j}=\beta_{j i}$ for every $i, j=1, \ldots, m, i \neq j$. We consider the following Coron-type problem:

$$
\left\{\begin{array}{ll}
-\Delta u_{i}=\mu_{i}\left|u_{i}\right|^{p-1} u_{i}+\sum_{j \neq i} \beta_{i j}\left|u_{j}\right|^{\frac{p+1}{2}}\left|u_{i}\right|^{\frac{p-3}{2}} u_{i} & \text { in } \Omega_{\varepsilon} \\
u_{i}=0 & \text { on } \partial \Omega_{\varepsilon},
\end{array} \quad i=1, \ldots, m,\right.
$$

where

$$
\Omega_{\varepsilon}:=\Omega \backslash \bigcup_{i=1}^{m} B_{r_{i} \varepsilon}\left(a_{i}\right)
$$

and $p:=2^{*}-1=(N+2) /(N-2)$.

For $\delta>0$ and $\xi \in \mathbb{R}^{N}$, we denote by $U_{\delta, \xi}$ the standard bubble

$$
U_{\delta, \xi}(x):=\alpha_{N}\left(\frac{\delta}{\delta^{2}+|x-\xi|^{2}}\right)^{\frac{N-2}{2}}
$$

where $\alpha_{N}>0$ is a suitable constant depending on the space dimension. It is well known (see [7, Corollary 8.2]) that the family $\left\{U_{\delta, \xi}: \delta>0, \xi \in \mathbb{R}^{N}\right\}$ contains all the solutions to the critical problem

$$
\begin{cases}-\Delta U=U^{p} & \text { in } \mathbb{R}^{N} \\ U>0 & \text { in } \mathbb{R}^{N} \\ U \in \mathcal{D}^{1,2}\left(\mathbb{R}^{N}\right), & \end{cases}
$$

where $\mathcal{D}^{1,2}\left(\mathbb{R}^{N}\right)$ is the completion of $\mathcal{C}_{c}^{\infty}\left(\mathbb{R}^{N}\right)$ with respect to the norm $\|u\|_{\mathcal{D}^{1,2}\left(\mathbb{R}^{N}\right)}:=\|\nabla u\|_{L^{2}\left(\mathbb{R}^{N}\right)}$. We consider the projection $P_{\varepsilon} U_{\delta, \xi}$ of $U_{\delta, \xi}$ into $H_{0}^{1}\left(\Omega_{\varepsilon}\right)$, i.e. the only solution to

$$
\begin{cases}-\Delta\left(P_{\varepsilon} U_{\delta, \xi}\right)=-\Delta U_{\delta, \xi}=U_{\delta, \xi}^{p} & \text { in } \Omega_{\varepsilon} \\ P_{\varepsilon} U_{\delta, \xi}=0 & \text { on } \partial \Omega_{\varepsilon}\end{cases}
$$

The first of our main results describe the situation where the components $u_{i}$ are all concentrating around different points.

Theorem 1.1. Let $N=4$, and let us suppose that $a_{i} \neq a_{j}$ for $i \neq j$. Then there exists $\bar{\varepsilon}, \bar{\beta}>0$ such that, if $\varepsilon \in(0, \bar{\varepsilon})$ and $-\infty<\beta_{i j}<\bar{\beta}$ for every $i \neq j$, then problem (1.4) has a fully nontrivial solution $\left(u_{1, \varepsilon}, \ldots, u_{m, \varepsilon}\right)$, where each $u_{i, \varepsilon}$ is positive and is concentrating around $a_{i}$ as $\varepsilon \rightarrow 0$.

To be precise, we have that

$$
u_{i, \varepsilon}=\mu_{i}^{-\frac{1}{p-1}} P_{\varepsilon} U_{\delta_{i}, \xi_{i}}+\phi_{i, \varepsilon}>0 \quad \text { in } \Omega_{\varepsilon},
$$

where $\delta_{i}=d_{i} \sqrt{\varepsilon}, \xi_{i}=a_{i}+\delta_{i} \tau_{i}$ for suitably chosen $d_{i}>0$ and $\tau_{i} \in \mathbb{R}^{N}$, and there exists $C>0$ (independent of $\varepsilon$ ) such that

$$
\left\|\phi_{i, \varepsilon}\right\|_{H_{0}^{1}\left(\Omega_{\varepsilon}\right)} \leq C \varepsilon^{\frac{N-2}{2}} \quad i=1, \ldots, m .
$$

If $N=3$, the same conclusion holds without any restriction on $\beta_{i j}$ (which can be also positive and large). 
Notice that in dimension $N=4$ we allow interactions of competitive type $\left(\beta_{i j}<0\right)$ or of weakly cooperative type $\left(0 \leq \beta_{i j}\right.$ small $)$ between the different components, while in dimension $N=3$ we have no restriction, and for the reason of this difference we refer to the forthcoming Remark 1.7

In Theorem 1.1 we proved the existence of solutions to (1.4) with all the components concentrating around different points. On the other hand, it is natural to wonder if it is possible to find solutions with several groups $G_{1}, \ldots, G_{q}$ of components such that:

- each component within a given group $G_{h}$ concentrate around a point $a_{h}$;

- the different groups concentrate around different points, i.e. $a_{h} \neq a_{k}$ if $h \neq k$.

The following theorem gives a positive answer to this question.

In what follow we focus on system (1.4) with 3 components in dimension $N=4$.

Theorem 1.2. Let $N=4, m=3$, and let us suppose that $a_{1}=a_{2} \neq a_{3}$. Let us suppose that either $-\sqrt{\mu_{1} \mu_{2}}<\beta_{12}<\min \left\{\mu_{1}, \mu_{2}\right\}$, or $\beta_{12}>\max \left\{\mu_{1}, \mu_{2}\right\}$. Then there exists $\bar{\varepsilon}, \bar{\beta}>0$ such that, if $\varepsilon \in(0, \bar{\varepsilon})$ and $-\infty<\beta_{13}, \beta_{23}<\bar{\beta}$, then problem (1.4) has a solution $\left(u_{1, \varepsilon}, u_{2, \varepsilon}, u_{3, \varepsilon}\right)$ where $u_{1, \varepsilon}$ and $u_{2, \varepsilon}$ are concentrating around $a_{1}$, while $u_{3, \varepsilon}$ is concentrating around $a_{3}$ as $\varepsilon \rightarrow 0$.

To be precise, let $c_{1}, c_{2}, c_{3}>0$ be defined by

$$
c_{1}^{2}=\frac{\beta_{12}-\mu_{2}}{\beta_{12}-\mu_{1} \mu_{2}}, \quad c_{2}^{2}=\frac{\beta_{12}-\mu_{1}}{\beta_{12}-\mu_{1} \mu_{2}}, \quad c_{3}=\mu_{3}^{-\frac{1}{2}} .
$$

We have that

$$
u_{i, \varepsilon}=c_{i} P_{\varepsilon} U_{\delta_{1}, \xi_{1}}+\phi_{i, \varepsilon}, \quad i=1,2, \quad u_{3, \varepsilon}=c_{3} P_{\varepsilon} U_{\delta_{3}, \xi_{3}}+\phi_{3, \varepsilon}
$$

where $\delta_{i}=d_{i} \sqrt{\varepsilon}, \xi_{i}=a_{i}+\delta_{i} \tau_{i}$ for suitably chosen $d_{i}>0$ and $\tau_{i} \in \mathbb{R}^{N}$, and there exists $C>0$ (independent of $\varepsilon$ ) such that

$$
\left\|\phi_{i, \varepsilon}\right\|_{H_{0}^{1}\left(\Omega_{\varepsilon}\right)} \leq C \varepsilon^{\frac{N-2}{2}} \quad i=1, \ldots, m .
$$

Moreover, there exists $\tilde{\beta} \leq \bar{\beta}$ such that if $-\infty<\beta_{i j}<\tilde{\beta}$ for every $i \neq j$, then $u_{i, \varepsilon}>0$ in $\Omega_{\varepsilon}$ for every $i$.

Notice that the interaction between the components $u_{1}$ and $u_{3}$ and between $u_{2}$ and $u_{3}$ is competitive or weakly cooperative, while the one between $u_{1}$ and $u_{2}$ is weakly competitive or cooperative (possibly with a large coupling parameter $\beta_{12}>0$ ).

Theorem 1.2 is the first result in the literature dealing with concentration of groups of components. As it will be clear from the proof, our method works in a much more general context with respect to the one considered in Theorem 1.2. One could both extend the result in $\mathbb{R}^{3}$, and (what is more important) consider more groups of components. This is the content of the forthcoming Theorem 1.3, for which we need some further notation. Let us consider system (1.4), and let $\beta_{i i}:=\mu_{i}$. $m$ if

For an arbitrary $1<q<m$, we say that a vector $\mathbf{l}=\left(l_{0}, \ldots, l_{q}\right) \in \mathbb{N}^{q+1}$ is a $q$-decomposition of

$$
0=l_{0}<l_{1}<\cdots<l_{q-1}<l_{q}=m
$$

given a $q$-decomposition $\mathbf{l}$ of $m$, we set, for $h=1, \ldots, q$,

$$
\begin{aligned}
& I_{h}:=\left\{i \in\{1, \ldots, m\}: l_{h-1}<i \leq l_{h}\right\}, \\
& \mathcal{K}:=\left\{(i, j) \in I_{h} \times I_{k} \text { with } h \neq k\right\} .
\end{aligned}
$$

This way, we have partitioned the set $\{1, \ldots, m\}$ into $q$ groups $I_{1}, \ldots, I_{q}$, and have consequently splitted the components into $q$ groups: $\left\{u_{i}: i \in I_{h}\right\}$. 
Let us consider the $q$ non-linear systems

$$
-\Delta U_{i}=\sum_{j \in I_{h}} \beta_{i j}\left|U_{i}\right|^{\frac{p-3}{2}}\left|U_{j}\right|^{\frac{p+1}{2}} U_{i} \quad \text { in } \mathbb{R}^{N}, i \in I_{h}, h=1, \ldots, q .
$$

Any such system has a solution $U_{i}=c_{i} U$, with $c_{i}>0$ for $i \in I_{h}$, and where $U=U_{1,0}$ is a standard bubble in $\mathbb{R}^{N}$ centered in 0 and with $\delta=1$, if and only if there exists a vector of positive numbers $\left(c_{1}, \ldots, c_{k}\right)$ such that

$$
\sum_{j=1, \ldots, k} \beta_{i j} c_{i}^{\frac{p-1}{2}} c_{j}^{\frac{p+1}{2}}=c_{i}, \quad i=1, \ldots, k
$$

Supposing that such a vector does exist, we linearize the $q$ systems $(1.9)$ in $\mathcal{D}^{1,2}\left(\mathbb{R}^{N}\right)$ around the solutions $\left(c_{l_{h-1}+1} U, \ldots, c_{l_{h}} U\right)$, obtaining

$$
-\Delta v_{i}=\left[\left(p \beta_{i i} c_{i}^{p-1}+\frac{p-1}{2} \sum_{\substack{j \in I_{h} \\ j \neq i}} \beta_{i j} c_{i}^{\frac{p-3}{2}} c_{j}^{\frac{p+1}{2}}\right) v_{i}+\frac{p+1}{2} \sum_{\substack{j \in I_{h} \\ j \neq i}} \beta_{i j} c_{i}^{\frac{p-1}{2}} c_{j}^{\frac{p-1}{2}} v_{j}\right] U^{p-1} \quad \text { in } \mathbb{R}^{N},
$$

for all $i \in I_{h}$, for all $h=1, \ldots, q$, with $v_{i} \in \mathcal{D}^{1,2}\left(\mathbb{R}^{N}\right)$.

Theorem 1.3. In the previous setting, let $N=4$, and let $a^{1}, \ldots, a^{q} \in \Omega$ with $a^{h} \neq a^{k}$ for $h \neq k$. Let us suppose that the $q$ systems $(1.9)$ have solutions $\left(c_{l_{h-1}+1}, \ldots, c_{l_{h}}\right), h=1, \ldots, q$, with $c_{i}>0$ for every $i$. Let us suppose also that each of the $q$ linearized systems (1.11) has $a(N+1)$-dimensional set of solutions. Then there exists $\bar{\varepsilon}, \bar{\beta}>0$ such that, if $\varepsilon \in(0, \bar{\varepsilon})$ and $-\infty<\beta_{i j}<\bar{\beta}$ for every $(i, j) \in \mathcal{K}$, then problem (1.4) has a solution $\left(u_{1, \varepsilon}, \ldots, u_{m, \varepsilon}\right)$, where $u_{1, \varepsilon}, \ldots, u_{l_{1}, \varepsilon}$ are concentrating around $a^{1}, u_{l_{1}+1, \varepsilon}, \ldots, u_{l_{2}, \varepsilon}$ are concentrating around $a^{2}, \ldots$, and $u_{l_{q-1}+1, \varepsilon}, \ldots, u_{l_{q}, \varepsilon}$ are concentrating around $a^{q}$ as $\varepsilon \rightarrow 0$.

To be precise, we have that for any $h=1, \ldots, q$

$$
u_{l_{h-1}+1, \varepsilon}=c_{l_{h-1}+1} P_{\varepsilon} U_{\delta_{h}, \xi_{h}}+\phi_{l_{h-1}+1, \varepsilon}, \quad \ldots \quad u_{l_{h}, \varepsilon}=c_{l_{h}} P_{\varepsilon} U_{\delta_{h}, \xi_{h}}+\phi_{l_{h}, \varepsilon},
$$

where $\delta_{h}=d_{h} \sqrt{\varepsilon}, \xi_{h}=a^{h}+\delta_{h} \tau_{h}$ for suitably chosen $d_{h}>0$ and $\tau_{h} \in \mathbb{R}^{N}$, and there exists $C>0$ (independent of $\varepsilon$ ) such that

$$
\left\|\phi_{i, \varepsilon}\right\|_{H_{0}^{1}\left(\Omega_{\varepsilon}\right)} \leq C \varepsilon^{\frac{N-2}{2}} \quad i=1, \ldots, m .
$$

Moreover, there exists $\tilde{\beta} \leq \bar{\beta}$ such that if $-\infty<\beta_{i j}<\tilde{\beta}$ for every $i \neq j$, then $u_{i, \varepsilon}>0$ in $\Omega_{\varepsilon}$ for every $i$.

In dimension $N=3$, the existence result holds, without any additional assumptions on the parameters $\beta_{i j}$ with $(i, j) \in \mathcal{K}$. Also, in this case we always obtain positive solutions.

Theorem 1.2 can be obtained applying Theorem 1.3 for the 3 components system in $\mathbb{R}^{4}$, considering the 2-decomposition of 3 given by $\mathbf{l}=(0,2,3)$. In this perspective, one has only to verify that the assumptions of Theorem 1.3 are satisfied, i.e. that the system

$$
\mu_{1} c_{1}^{2}+\beta_{12} c_{2}^{2}=1, \quad \mu_{2} c_{2}^{2}+\beta_{12} c_{1}^{2}=1,
$$

has a solution with $c_{1}, c_{2}>0$, and that the linearized problem

$$
-\Delta v_{i}=\left(3 \mu_{i} c_{i}^{2}+\beta_{i j} c_{j}^{2}\right) U^{2} v_{i}+2 \beta_{i j} c_{i} c_{j} U^{2} v_{j} \quad \text { in } \mathbb{R}^{4}, i=1,2, j \neq i
$$

has a 5 -dimensional set of solutions.

Nevertheless, for the sake of simplicity and brevity, we directly write down the proof of Theorem 1.2, without deriving it as corollary of Theorem 1.2, and we omit the proof of Theorem 1.3. The passage from the particular situation described in Theorem 1.2 to the general setting considered in 
Theorem 1.3 creates additional difficulties only from the technical and notational points of view, and once that the main idea are understood, the interested reader can easily fill the details.

Rather, one point which we want to stress is that the assumptions of Theorem 1.3 only regard the data of the problem, and in general are not too difficult to check, as shown in the following proposition.

Given the number of components $m$, the number of groups $1<q<m$, a $q$-decomposition $\mathbf{l}=\left(l_{0}, \ldots, l_{q}\right)$ of $m$, and the coupling parameters $\beta_{i j}, i, j=1, \ldots, m$, let us consider the $q$ matrices

$$
\mathcal{B}_{h}:=\left(\beta_{i j}\right)_{(i, j) \in I_{h} \times I_{h}} \quad h=1, \ldots, q .
$$

Notice that if $l_{h}-l_{h-1}=1$ for some $i$, then $I_{h}$ is a singleton and the corresponding $\mathcal{B}_{h}$ is simply given by the real number $\beta_{i i}, i \in I_{h}$.

Conditions on $\mathcal{B}_{h}$ ensuring the existence of solutions $\left(c_{l_{h-1}+1}, \ldots, c_{l_{h}}\right), h=1, \ldots, q$, for the algebraic problem (1.10) are given in [3, Section 2] or [27, Section 4].

So, let us suppose that such $\left(c_{l_{h-1}+1}, \ldots, c_{l_{h}}\right), h=1, \ldots, q$, do exist.

Proposition 1.4. Let $N=4$. In the previous setting, and using the notation of Theorem 1.3, let us suppose that for any $h=1, \ldots, q$ we have

the matrix $\mathcal{B}_{h}$ is invertible and has only positive elements.

Then each of the $h$ linearized systems (1.11) has a $(N+1)$-dimensional set of solutions.

Combining then the results in $[3,27]$ with Proposition 1.4, Theorem 1.3 permits to prove existence of a bunch of solutions, with arbitrary number $q$ of groups (each of them having an arbitrary number of components) concentrating around $q$ different points.

The proofs of Theorems 1.1 and 1.2 rest upon a finite-dimensional reduction, and will be the object of the next sections. Before proceeding, we conclude the introduction with some comments.

Remark 1.5. We point out that, both in Theorems 1.1, 1.2 and 1.3, we can deal with systems with mixed cooperation and competition (that is, we can deal with systems where some $\beta_{i j}$ is positive, and some other is negative). This is particularly interesting since the mixed coupling critical case was completely open, and also for subcritical problems has been investigated only in few recent contributions (see $[6,25,26,28,29]$ ).

Remark 1.6. Regarding the positivity in dimension $N=4$, it is natural to think that in Theorems 1.2 and 1.3 we have a positive solution without any additional assumptions on $\beta_{i j}$. Indeed, any component $u_{i, \varepsilon}$ is a superposition of positive function and small perturbation term. If the positive part of $\beta_{i j}$ is small for every $i \neq j$, then a short rigorous proof of the positivity can be given arguing as in [23]. If on the other hand some $\beta_{i j}$ is allowed to be large, such proof does not work and one is forced to approach the problem with finer (and much longer) techniques, such as careful $L^{\infty}$-estimates on the error $\phi_{i, \varepsilon}$ (see for instance [17, Section 8] for related computations). We decided to not insist on this point for the sake of brevity.

Remark 1.7. As it emerges from Theorem 1.1, in $\mathbb{R}^{4}$ we have to suppose extra-conditions on $\beta_{i j}$ in order to have existence of fully nontrivial positive solutions, with respect to the 3-dimensional problem. This is somehow natural, and is related to the particular shape of the cubic system (in dimension $N=4$ we have $p=3$ ) which makes possible to prove non-existence results (for instance, one can repeat word by word the proof of Theorem 3-(iii) in [27] to rule out the existence of positive solutions under some assumptions on the parameters). This difference between the dimension $N=4$ and $N \neq 4$ was already observed in [10]. 
The extra-condition $-\infty<\beta_{i j}<\bar{\beta}$ with $\bar{\beta}$ small enough was already considered in [23], and plays the same role in our proof and the one in [23]; it enters in the analysis of the linearization of (1.4) and in the proof of the positivity of the solutions. It is interesting that this assumption is not needed in dimension 3 .

Regarding the higher dimensional case $N \geq 5$, the main obstruction for our method is represented by the lack of regularity of the interaction term $\left|u_{j}\right|^{\frac{p+1}{2}}\left|u_{i}\right|^{\frac{p-3}{2}} u_{i}$, which is not of class $\mathcal{C}^{1}$. This creates several additional difficulties in dealing with a linearization of (1.4), difficulties which were already observed in $[15,23]$.

Remark 1.8. Both Theorems 1.1 and 1.2 regard the case when at least two components concentrate around different points, and hence it is natural to wonder what happens if we search for solutions to (1.4) with all the components concentrating around the same point. Under appropriate assumptions on $\beta_{i j}$, this case is actually much simpler to deal with, since one can obtain a solution with all the components proportional among each other, reducing system (1.4) to the scalar problem (1.6). For instance, for a system with 2 components in a domain $\Omega \subset \mathbb{R}^{4}$, it is not difficult to check that if (1.6) has a solution $w$ (positive or sign-changing, concentrating around one or more points), and if

$$
\text { either }-\sqrt{\mu_{1} \mu_{2}}<\beta_{12}<\min \left\{\mu_{1}, \mu_{2}\right\} \text {, or } \beta_{12}>\max \left\{\mu_{1}, \mu_{2}\right\},
$$

then (1.4) in $\Omega$ has a solution of type $\left(u_{1}, u_{2}\right)=\left(c_{1} w, c_{2} w\right)$, with $c_{1}, c_{2}$ as in Theorem 1.2. In particular, we have:

- Bahri-Coron-type result: if $\Omega \subset \mathbb{R}^{4}$ has non-trivial topology and (1.12) is in force, then the critical system (1.4) has a positive solutions.

- Solutions concentrating around the same point in domains with one shrinking hole: if $a \in \Omega \subset \mathbb{R}^{4}, \Omega_{\varepsilon}=\Omega \backslash B_{\varepsilon}(a)$, and (1.12) holds true, then (1.4) has a family of positive solutions with $u_{1, \varepsilon}$ and $u_{2, \varepsilon}$ concentrating around the same point with the same speed.

- Multipeak solutions and multiplicity results, extending $[11,12,14,18,21,24]$ as in the previous points.

Similar results in higher or lower dimension can be obtained using the existence of $\left(k_{0}, l_{0}\right)$ as in [10, Theorem 1.1]. In the same way, in order to deal with more than 2 components one can use the results in [3, Section 2] or [27, Section 4].

Remark 1.9. In our results, we focus on domains $\Omega_{\varepsilon}$ with radially symmetric holes $B_{r_{i} \varepsilon}\left(a_{i}\right)$. It is not difficult to check that we can treat also the case of non-symmetric holes. In such a situation, we could repeat our argument essentially word by word, simply replacing Lemma A.1 with Lemma 1.1 in [19]; this introduces some technical complications which we preferred to avoid.

Structure of the paper. The proof of Theorem 1.1 is contained in Sections 2-4, while the proof of Theorem 1.2 is given in 5. Both are based upon the Lyapunov-Schmidt finite dimensional reduction method, which in the context of systems was already adopted in [23] to deal with the Brezis-Nirenberg-type problem. The proof of Proposition 1.4 is given in Section 6 .

In Section 2 we set up the reduction scheme, splitting system (1.4) in two new systems of $m$ equations, one of them living in finite dimension. The infinite dimensional problem is then treated in Section 3 via a fixed point argument, while the finitely-reduced problem is the object of Section 4.

Since Theorem 1.2 shares the same structure and many passages with that of Theorem 1.1, we put particular emphasis on the main differences.

As usual when dealing with a perturbation approach, many proofs contains very long computations. In order to keep the presentation as smooth as possible, we collect them in several 
appendixes, so that the reader can easily understand the main strategy behind each proof, and check the details in a second time.

Notation and preliminary results. We recall that with $U_{\delta, \xi}$ and $P_{\varepsilon} U_{\delta, \xi}$ we denote the standard bubble and its projection in $H_{0}^{1}\left(\Omega_{\varepsilon}\right)$, defined in (1.5) and (1.7). We shall use many times the fact that $0 \leq P_{\varepsilon} U_{\delta, \xi} \leq U_{\delta, \xi}$, which is a simple consequence of the maximum principle.

Since $\left\{U_{\delta, \xi}\right\}$ is the set of all the solutions to (1.6), it is easy to check that any solution to

$$
\begin{cases}-\Delta U=\mu U^{p} & \text { in } \mathbb{R}^{N} \\ U>0 & \text { in } \mathbb{R}^{N} \\ U \in \mathcal{D}^{1,2}\left(\mathbb{R}^{N}\right) & \end{cases}
$$

with $\mu>0$ is given by $\mu^{-\frac{1}{p-1}} U_{\delta, \xi}$, for some $\delta>0$ and $\xi \in \mathbb{R}^{N}$.

Coming back to problem (1.6), we recall some properties of the linearized equation

$$
-\Delta \phi=p U_{\delta, \xi}^{p-1} \phi \quad \text { in } \mathbb{R}^{N}, \quad \phi \in \mathcal{D}^{1,2}\left(\mathbb{R}^{N}\right) .
$$

It is clear that there are $N+1$ solutions given by

$$
\begin{aligned}
& \psi_{\delta, \xi}^{0}:=\frac{\partial U_{\delta, \xi}}{\partial \delta}=\alpha_{N}\left(\frac{N-2}{2}\right) \delta^{\frac{N-4}{2}} \frac{|x-\xi|^{2}-\delta^{2}}{\left(\delta^{2}+|x-\xi|^{2}\right)^{\frac{N}{2}}} \\
& \psi_{\delta, \xi}^{h}:=\frac{\partial U_{\delta, \xi}}{\partial \xi_{\ell}}=\alpha_{N}(N-2) \delta^{\frac{N-2}{2}} \frac{x_{\ell}-\xi_{\ell}}{\left(\delta^{2}+|x-\xi|^{2}\right)^{\frac{N}{2}}}, \quad h=1, \ldots, N .
\end{aligned}
$$

By [4, Lemma A.1], these functions span the set of solutions to (1.13).

We consider the projections $P_{\varepsilon} \psi_{\delta, \xi}^{h}$ of $\psi_{\delta, \xi}^{h}(h=0, \ldots, N)$ into $H_{0}^{1}\left(\Omega_{\varepsilon}\right)$, i.e.

$$
\begin{cases}-\Delta\left(P_{\varepsilon} \psi_{\delta, \xi}^{h}\right)=-\Delta \psi_{\delta, \xi}^{h}=p U_{\delta, \xi}^{p-1} \psi_{\delta, \xi}^{h} & \text { in } \Omega_{\varepsilon} \\ P_{\varepsilon} \psi_{\delta, \xi}^{h}=0 & \text { on } \partial \Omega_{\varepsilon} .\end{cases}
$$

Notice that the derivative commutes with the projection $P_{\varepsilon}$, in the sense that $P_{\varepsilon} \psi_{\delta, \xi}^{h}=\partial_{h}\left(P_{\varepsilon} U_{\delta, \xi}\right)$, where $\partial_{h}$ denotes the partial derivative with respect to $\xi_{h}$ if $h=1, \ldots, N$, and $\partial_{0}$ denotes the partial derivative with respect to $\delta$.

We denote by $G(x, y)$ the Green function of $-\Delta$ with Dirichlet boundary condition in $\Omega$, that is the function satisfying, for fixed $y \in \Omega$,

$$
\begin{cases}-\Delta_{x} G(\cdot, y)=\delta_{y} & \text { in } \Omega \\ G(\cdot, y)=0 & \text { on } \partial \Omega\end{cases}
$$

where $\delta_{y}$ is the Dirac delta centered in $y$. It is well known that the Green function can be decomposed as

$$
G(x, y)=\frac{1}{N(N-2) \omega_{N}|x-y|^{N-2}}+H(x, y),
$$

where $\omega_{N}$ is the volume of the unit ball in $\mathbb{R}^{N}$, and $H(x, y)$ is the regular part of the Green function, defined for fixed $y \in \Omega$ as the solution to

$$
\begin{cases}-\Delta_{x} H(\cdot, y)=0 & \text { in } \Omega \\ H(\cdot, y)=\frac{1}{N(N-2) \omega_{N}|\cdot-y|^{N-2}} & \text { on } \partial \Omega,\end{cases}
$$

Finally, we denote the standard inner product and norms in $H_{0}^{1}(\Omega)$ by

$$
\langle u, v\rangle_{H_{0}^{1}(\Omega)}:=\int_{\Omega} \nabla u \cdot \nabla v, \quad\|u\|_{H_{0}^{1}(\Omega)}:=(\langle u, u\rangle)^{\frac{1}{2}},
$$


and the $L^{q}$-norm $(q \geq 1)$ by $|\cdot|_{L^{q}(\Omega)}$. When there is no possibility of misunderstanding, we shall often adopt the simplified notations $\|\cdot\|$ and $|\cdot|_{q}$, for the sake of brevity.

In the rest of the paper $C$ always denotes a positive constant which can depend on the dimension $N$, on the data $\mu_{i}$ and $\beta_{i j}$, but not on $\varepsilon$. In two steps we will need to point out that a constant $C$ does not depend on $\beta_{i j}$; in such cases we will explicitly write it. The exact value of $C$ can change from line to line.

In many cases, to estimate some quantity involving $P_{\varepsilon} U_{\delta, \xi}$ or $P_{\varepsilon} \psi_{\delta, \xi}^{i}$, it will be necessary to approximate the projections with the original functions, carefully controlling the difference. These kind of results are mainly collected in Appendix A.

\section{Proof of Theorem 1.1: The Reduction SCheme}

Being interested in positive solutions, instead of problem (1.4) we consider

$$
\left\{\begin{array}{ll}
-\Delta u_{i}=\mu_{i} f\left(u_{i}\right)+\sum_{j \neq i} \beta_{i j}\left|u_{j}\right|^{\frac{p+1}{2}}\left|u_{i}\right|^{\frac{p-3}{2}} u_{i} & \text { in } \Omega_{\varepsilon} \\
u_{i}=0 & \text { on } \partial \Omega_{\varepsilon},
\end{array} \quad i=1, \ldots, m,\right.
$$

where $f(s):=|s|^{p-1} s^{+}$. We shall see that this replacement makes possible to prove positivity of the solutions in a very simple way, under the assumptions described by the main theorems.

Let $i: H_{0}^{1}\left(\Omega_{\varepsilon}\right) \rightarrow L^{2^{*}}\left(\Omega_{\varepsilon}\right)$ be the canonical Sobolev embedding. We consider the adjoint operator $i^{*}: L^{\frac{2 N}{N+2}}\left(\Omega_{\varepsilon}\right) \rightarrow H_{0}^{1}\left(\Omega_{\varepsilon}\right)$, characterized by

$$
i^{*}(u)=v \quad \Longleftrightarrow\left\{\begin{array}{l}
-\Delta v=u \\
v \in H_{0}^{1}(\Omega)
\end{array} \text { in } \Omega\right. \text { (in weak sense) }
$$

It is well known that $i^{*}$ is a continuous operator, and using it we can rewrite (2.1) as

$$
u_{i}=i^{*}\left(\mu_{i} f\left(u_{i}\right)+\sum_{j \neq i} \beta_{i j}\left|u_{j}\right|^{\frac{p+1}{2}}\left|u_{i}\right|^{\frac{p-3}{2}} u_{i}\right) \text {. }
$$

We search for solutions as perturbation of bubbles centered at different points: let $\eta \in(0,1)$ be small, and let

$$
X_{\eta}:=\left\{(\mathbf{d}, \boldsymbol{\tau}) \in \mathbb{R}^{m} \times\left(\mathbb{R}^{N}\right)^{m}: \eta<d_{i}<\eta^{-1},\left|\tau_{i}\right|<\eta^{-1}\right\} .
$$

Our ansatz is that

$$
u_{i}=\mu_{i}^{-\frac{1}{p-1}} P_{\varepsilon} U_{\delta_{i}, \xi_{i}}+\phi_{i}
$$

where for some $(\mathbf{d}, \boldsymbol{\tau})=\left(d_{1}, \ldots, d_{m}, \tau_{1}, \ldots, \tau_{m}\right) \in X_{\eta}$ we have

$$
\delta_{i}:=d_{i} \sqrt{\varepsilon}, \quad \xi_{i}:=a_{i}+d_{i} \sqrt{\varepsilon} \tau_{i} .
$$

We stress that the quantity $\eta$ will always be fixed and small, while the unknowns will be $d_{i}>0$, $\tau_{i} \in \mathbb{R}^{N}$, and $\phi_{i} \in H_{0}^{1}\left(\Omega_{\varepsilon}\right)$.

Remark 2.1. Once that $\eta>0$ is fixed, we observe that

$$
\delta_{i}=O\left(\varepsilon^{\frac{1}{2}}\right) \quad \text { and } \quad \varepsilon=O\left(\delta_{i}^{2}\right) \quad \forall i=1, \ldots, m
$$

as $\varepsilon \rightarrow 0$. 
Plugging ansatz (2.4) into (2.2), our problem is transformed in the research of $d_{i}, \tau_{i}$, and $\phi_{i}$ such that

$$
\begin{aligned}
\mu_{i}^{-\frac{1}{p-1}} P_{\varepsilon} & U_{\delta_{i}, \xi_{i}}+\phi_{i}=i^{*}\left[\mu_{i} f\left(\mu_{i}^{-\frac{1}{p-1}} P_{\varepsilon} U_{\delta_{i}, \xi_{i}}+\phi_{i}\right)\right. \\
& \left.+\sum_{j \neq i} \beta_{i j}\left|\mu_{j}^{-\frac{1}{p-1}} P_{\varepsilon} U_{\delta_{j}, \xi_{j}}+\phi_{j}\right|^{\frac{p+1}{2}}\left|\mu_{i}^{-\frac{1}{p-1}} P_{\varepsilon} U_{\delta_{i}, \xi_{i}}+\phi_{i}\right|^{\frac{p-3}{2}}\left(\mu_{i}^{-\frac{1}{p-1}} P_{\varepsilon} U_{\delta_{i}, \xi_{i}}+\phi_{i}\right)\right]
\end{aligned}
$$

for $i=1, \ldots, m$, with each equality which takes place in $H_{0}^{1}\left(\Omega_{\varepsilon}\right)$. To proceed, the idea is then to split the space into two orthogonal subspaces, one of them having finite dimension. To be precise, for $\varepsilon>0, d_{1}, \ldots, d_{m}>0$, and $\tau_{1}, \ldots, \tau_{m} \in \mathbb{R}^{N}$, we define

$$
K_{i}=K_{d_{i}, \tau_{i}, \varepsilon}:=\operatorname{span}\left\{P_{\varepsilon} \psi_{\delta_{i}, \xi_{i}}^{h}: h=0, \ldots, N\right\}, \quad \mathbf{K}_{\mathbf{d}, \boldsymbol{\tau}, \varepsilon}:=K_{1} \times \cdots \times K_{m}
$$

(recall that $\delta_{i}$ and $\xi_{i}$ are determined by $d_{i}, \tau_{i}$ and $\varepsilon$ through the ansatz (2.5)). Notice that $K_{\mathbf{d}, \boldsymbol{\tau}, \varepsilon}^{\perp}=$ $K_{1}^{\perp} \times \cdots \times K_{m}^{\perp}$.

If $\Pi_{i}=\Pi_{\delta_{i}, \xi_{i}, \varepsilon}$ (resp. $\left.\Pi_{i}^{\perp}=\Pi_{\delta_{i}, \xi_{i}, \varepsilon}^{\perp}\right)$ denotes the orthogonal projection $H_{0}^{1}\left(\Omega_{\varepsilon}\right) \rightarrow K_{i}$ (resp. $\left.H_{0}^{1}\left(\Omega_{\varepsilon}\right) \rightarrow K_{i}^{\perp}\right)$, then $(2.6)$ can be further rewritten as a system of $2 m$ equations

$$
\begin{aligned}
& \Pi_{i}\left(\mu_{i}^{-\frac{1}{p-1}} P_{\varepsilon} U_{\delta_{i}, \xi_{i}}+\phi_{i}\right)=\left(\Pi_{i} \circ i^{*}\right)\left[\mu_{i} f\left(\mu_{i}^{-\frac{1}{p-1}} P_{\varepsilon} U_{\delta_{i}, \xi_{i}}+\phi_{i}\right)\right. \\
& \left.\quad+\sum_{j \neq i} \beta_{i j}\left|\mu_{j}^{-\frac{1}{p-1}} P_{\varepsilon} U_{\delta_{j}, \xi_{j}}+\phi_{j}\right|^{\frac{p+1}{2}}\left|\mu_{i}^{-\frac{1}{p-1}} P_{\varepsilon} U_{\delta_{i}, \xi_{i}}+\phi_{i}\right|^{\frac{p-3}{2}}\left(\mu_{i}^{-\frac{1}{p-1}} P_{\varepsilon} U_{\delta_{i}, \xi_{i}}+\phi_{i}\right)\right]
\end{aligned}
$$

and

$$
\begin{aligned}
& \Pi_{i}^{\perp}\left(\mu_{i}^{-\frac{1}{p-1}} P_{\varepsilon} U_{\delta_{i}, \xi_{i}}+\phi_{i}\right)=\left(\Pi_{i}^{\perp} \circ i^{*}\right)\left[\mu_{i} f\left(\mu_{i}^{-\frac{1}{p-1}} P_{\varepsilon} U_{\delta_{i}, \xi_{i}}+\phi_{i}\right)\right. \\
& \left.\quad+\sum_{j \neq i} \beta_{i j}\left|\mu_{j}^{-\frac{1}{p-1}} P_{\varepsilon} U_{\delta_{j}, \xi_{j}}+\phi_{j}\right|^{\frac{p+1}{2}}\left|\mu_{i}^{-\frac{1}{p-1}} P_{\varepsilon} U_{\delta_{i}, \xi_{i}}+\phi_{i}\right|^{\frac{p-3}{2}}\left(\mu_{i}^{-\frac{1}{p-1}} P_{\varepsilon} U_{\delta_{i}, \xi_{i}}+\phi_{i}\right)\right]
\end{aligned}
$$

$i=1, \ldots, m$.

The proof of Theorem 1.1 consists of two main steps: first, for fixed $\varepsilon, d_{1}, \ldots, d_{m}$, and $\tau_{1}, \ldots, \tau_{m}$ we solve the $m$-equations system $(2.8)$, finding $\left(\phi_{1}^{\mathbf{d}, \boldsymbol{\tau}, \varepsilon}, \ldots, \phi_{m}^{\mathbf{d}, \boldsymbol{\tau}, \varepsilon}\right) \in \mathbf{K}_{\mathbf{d}, \boldsymbol{\tau}, \varepsilon}^{\perp}$. Plugging this choice of $\boldsymbol{\phi}$ into (2.7), we obtain a finite dimensional problem in the unknowns $\mathbf{d}$ and $\boldsymbol{\tau}$, which will be solved in a second step for any $\varepsilon>0$ sufficiently small.

\section{The equations in $\mathbf{K}_{\mathbf{d}, \boldsymbol{\tau}, \varepsilon}^{\perp}$}

In this section we study the solvability of (2.8). In a functional analytic perspective, (2.8) reads

$$
L_{\mathbf{d}, \boldsymbol{\tau}, \varepsilon}^{i}(\phi)=N_{\mathbf{d}, \boldsymbol{\tau}, \varepsilon}^{i}(\phi)+R_{\mathbf{d}, \boldsymbol{\tau}, \varepsilon}^{i}
$$


where $L$ stays for the linear part

$$
\begin{aligned}
L_{\mathbf{d}, \boldsymbol{\tau}, \varepsilon}^{i}(\phi) & =\Pi_{i}^{\perp}\left\{\phi_{i}-i^{*}\left[\mu_{i} f^{\prime}\left(\mu_{i}^{-\frac{1}{p-1}} P_{\varepsilon} U_{\delta_{i}, \xi_{i}}\right) \phi_{i}\right.\right. \\
& +\frac{p-1}{2} \sum_{j \neq i} \beta_{i j}\left(\mu_{j}^{-\frac{1}{p-1}} P_{\varepsilon} U_{\delta_{j}, \xi_{j}}\right)^{\frac{p+1}{2}}\left(\mu_{i}^{-\frac{1}{p-1}} P_{\varepsilon} U_{\delta_{i}, \xi_{i}}\right)^{\frac{p-3}{2}} \phi_{i} \\
& \left.\left.+\frac{p+1}{2} \sum_{j \neq i} \beta_{i j}\left(\mu_{j}^{-\frac{1}{p-1}} P_{\varepsilon} U_{\delta_{j}, \xi_{j}}\right)^{\frac{p-1}{2}}\left(\mu_{i}^{-\frac{1}{p-1}} P_{\varepsilon} U_{\delta_{i}, \xi_{i}}\right)^{\frac{p-1}{2}} \phi_{j}\right]\right\},
\end{aligned}
$$

$N$ stays for the nonlinear part

$$
\begin{aligned}
N_{\mathbf{d}, \boldsymbol{\tau}, \varepsilon}^{i}( & \phi)= \\
& \Pi_{i}^{\perp} \circ i^{*}\left[\mu_{i} f\left(\mu_{i}^{-\frac{1}{p-1}} P_{\varepsilon} U_{\delta_{i}, \xi_{i}}+\phi_{i}\right)-\mu_{i} f\left(\mu_{i}^{-\frac{1}{p-1}} P_{\varepsilon} U_{\delta_{i}, \xi_{i}}\right)-\mu_{i} f^{\prime}\left(\mu_{i}^{-\frac{1}{p-1}} P_{\varepsilon} U_{\delta_{i}, \xi_{i}}\right) \phi_{i}\right. \\
& +\sum_{j \neq i} \beta_{i j}\left|\mu_{j}^{-\frac{1}{p-1}} P_{\varepsilon} U_{\delta_{j}, \xi_{j}}+\phi_{j}\right|^{\frac{p+1}{2}}\left|\mu_{i}^{-\frac{1}{p-1}} P_{\varepsilon} U_{\delta_{i}, \xi_{i}}+\phi_{i}\right|^{\frac{p-3}{2}}\left(\mu_{i}^{-\frac{1}{p-1}} P_{\varepsilon} U_{\delta_{i}, \xi_{i}}+\phi_{i}\right) \\
& -\sum_{j \neq i} \beta_{i j}\left(\mu_{j}^{-\frac{1}{p-1}} P_{\varepsilon} U_{\delta_{j}, \xi_{j}}\right)^{\frac{p+1}{2}}\left(\mu_{i}^{-\frac{1}{p-1}} P_{\varepsilon} U_{\delta_{i}, \xi_{i}}\right)^{\frac{p-1}{2}} \\
& -\frac{p-1}{2} \sum_{j \neq i} \beta_{i j}\left(\mu_{j}^{-\frac{1}{p-1}} P_{\varepsilon} U_{\delta_{j}, \xi_{j}}\right)^{\frac{p+1}{2}}\left(\mu_{i}^{-\frac{1}{p-1}} P_{\varepsilon} U_{\delta_{i}, \xi_{i}}\right)^{\frac{p-3}{2}} \phi_{i} \\
& \left.-\frac{p+1}{2} \sum_{j \neq i} \beta_{i j}\left(\mu_{j}^{-\frac{1}{p-1}} P_{\varepsilon} U_{\delta_{j}, \xi_{j}}\right)^{\frac{p-1}{2}}\left(\mu_{i}^{-\frac{1}{p-1}} P_{\varepsilon} U_{\delta_{i}, \xi_{i}}\right)^{\frac{p-1}{2}} \phi_{j}\right],
\end{aligned}
$$

and $R$ is the remainder term

$$
\begin{aligned}
R_{\mathbf{d}, \boldsymbol{\tau}, \varepsilon}^{i}=\Pi_{i}^{\perp}\{ & -\mu_{i}^{-\frac{1}{p-1}} P_{\varepsilon} U_{\delta_{i}, \xi_{i}} \\
& \left.+i^{*}\left[\mu_{i} f\left(\mu_{i}^{-\frac{1}{p-1}} P_{\varepsilon} U_{\delta_{i}, \xi_{i}}\right)+\sum_{j \neq i} \beta_{i j}\left(\mu_{j}^{-\frac{1}{p-1}} P_{\varepsilon} U_{\delta_{j}, \xi_{j}}\right)^{\frac{p+1}{2}}\left(\mu_{i}^{-\frac{1}{p-1}} P_{\varepsilon} U_{\delta_{i}, \xi_{i}}\right)^{\frac{p-1}{2}}\right]\right\} \\
= & \Pi_{i}^{\perp} \circ i^{*}\left[\mu_{i}^{-\frac{1}{p-1}}\left(P_{\varepsilon} U_{\delta_{i}, \xi_{i}}^{p}-U_{\delta_{i}, \xi_{i}}^{p}\right)+\sum_{j \neq i} \beta_{i j}\left(\mu_{j}^{-\frac{1}{p-1}} P_{\varepsilon} U_{\delta_{j}, \xi_{j}}\right)^{\frac{p+1}{2}}\left(\mu_{i}^{-\frac{1}{p-1}} P_{\varepsilon} U_{\delta_{i}, \xi_{i}}\right)^{\frac{p-1}{2}}\right],
\end{aligned}
$$

where the last equality is a consequence of the definitions of $i^{*}$ and of $f$.

For future convenience, we also define

$$
\mathbf{L}_{\mathbf{d}, \boldsymbol{\tau}, \varepsilon}:=\left(L_{\mathbf{d}, \boldsymbol{\tau}, \varepsilon}^{1}, \ldots, L_{\mathbf{d}, \boldsymbol{\tau}, \varepsilon}^{m}\right): \mathbf{K}_{\mathbf{d}, \boldsymbol{\tau}, \varepsilon}^{\perp} \rightarrow \mathbf{K}_{\mathbf{d}, \boldsymbol{\tau}, \varepsilon}^{\perp},
$$

and $\mathbf{R}_{\mathbf{d}, \boldsymbol{\tau}, \varepsilon}$ and $\mathbf{N}_{\mathbf{d}, \boldsymbol{\tau}, \varepsilon}$ in an analogue way.

The main result of this section is the following:

Proposition 3.1. Let $N=4$. For every $\eta>0$ small enough there exists $\bar{\beta}, \varepsilon_{0}>0$ small, and $C>0$, such that if $\varepsilon \in\left(0, \varepsilon_{0}\right)$, and $-\infty<\beta_{i j}<\bar{\beta}$ for every $i \neq j$, then for any $(\mathbf{d}, \boldsymbol{\tau}) \in X_{\eta}$ (see (2.3)) there exists a unique function $\phi^{\mathbf{d}, \boldsymbol{\tau}, \varepsilon} \in K_{\mathbf{d}, \boldsymbol{\tau}, \varepsilon}^{\perp}$ solving the equation

$$
\mathbf{L}_{\mathbf{d}, \boldsymbol{\tau}, \varepsilon}(\phi)=\mathbf{R}_{\mathbf{d}, \boldsymbol{\tau}, \varepsilon}+\mathbf{N}_{\mathbf{d}, \boldsymbol{\tau}, \varepsilon}(\phi)
$$


and satisfying

$$
\left\|\phi^{\mathbf{d}, \boldsymbol{\tau}, \varepsilon}\right\|_{H_{0}^{1}\left(\Omega_{\varepsilon}\right)} \leq C \varepsilon^{\frac{N-2}{2}} .
$$

Furthermore, the map $(\varepsilon, \mathbf{d}, \boldsymbol{\tau}) \mapsto \boldsymbol{\phi}^{\mathbf{d}, \boldsymbol{\tau}, \varepsilon}$ is of class $\mathcal{C}^{1}$, and

$$
\left\|\nabla_{(\mathbf{d}, \boldsymbol{\tau})} \phi^{\mathbf{d}, \boldsymbol{\tau}, \varepsilon}\right\|_{H_{0}^{1}\left(\Omega_{\varepsilon}\right)} \leq C \varepsilon^{\frac{N-2}{2}} .
$$

If $N=3$, the same conclusion holds without any restriction on $\beta_{i j}$.

The proof of the proposition takes the rest of this section, and is divided into several intermediate lemmas.

3.1. Study of the linear part. As a first step, it is important to understand the solvability of the linear problem associated to (3.1), i.e.

$$
L_{\mathbf{d}, \boldsymbol{\tau}, \varepsilon}^{i}(\phi)=f_{i}, \quad \text { with } \quad f_{i} \in K_{d_{i}, \tau_{i}, \varepsilon}^{\perp} .
$$

Lemma 3.2. Let $N=4$. For every $\eta>0$ small enough there exists $\bar{\beta}, \varepsilon_{0}>0$ small, and $C>0$, such that if $\varepsilon \in\left(0, \varepsilon_{0}\right)$, and $-\infty<\beta_{i j}<\bar{\beta}$ for every $i \neq j$, then

$$
\left\|\mathbf{L}_{\mathbf{d}, \boldsymbol{\tau}, \varepsilon}(\boldsymbol{\phi})\right\|_{H_{0}^{1}\left(\Omega_{\varepsilon}\right)} \geq C\|\phi\|_{H_{0}^{1}\left(\Omega_{\varepsilon}\right)} \quad \forall \boldsymbol{\phi} \in H_{0}^{1}\left(\Omega_{\varepsilon}, \mathbb{R}^{m}\right)
$$

for every $(\mathbf{d}, \boldsymbol{\tau}) \in X_{\eta}$. Moreover, $\mathbf{L}_{\mathbf{d}, \boldsymbol{\tau}, \varepsilon}$ is invertible in $\mathbf{K}_{\mathbf{d}, \boldsymbol{\tau}, \varepsilon}^{\perp}$, with continuous inverse. If $N=3$, the same conclusion holds true without restrictions on $\beta_{i j}$.

Proof. The long proof proceed by contradiction. Let us suppose that there exist sequences

$$
\left\{\varepsilon_{n}\right\} \subset \mathbb{R}^{+}, \varepsilon_{n} \rightarrow 0,\left\{\left(\mathbf{d}_{n}, \boldsymbol{\tau}_{n}\right)\right\} \subset X_{\eta},\left\{\boldsymbol{\phi}_{n}\right\} \subset K_{1, n}^{\perp} \times \cdots \times K_{m, n}^{\perp}
$$

such that

$$
\left\|\phi_{n}\right\|_{H_{0}^{1}\left(\Omega_{\varepsilon_{n}}\right)}=1 \quad \text { and } \quad\left\|\mathbf{L}_{n}\left(\phi_{n}\right)\right\|_{H_{0}^{1}\left(\Omega_{\varepsilon_{n}}\right)} \rightarrow 0
$$

as $n \rightarrow \infty$, where we wrote $K_{i, n}:=K_{d_{i, n}, \tau_{i, n}, \varepsilon_{n}}$ and $\mathbf{L}_{n}:=\mathbf{L}_{\mathbf{d}_{n}, \boldsymbol{\tau}_{n}, \varepsilon_{n}}$ for short. In the same spirit in this proof we write $P_{n}:=P_{\varepsilon_{n}}, U_{i, n}:=U_{\delta_{i, n}, \xi_{i, n}}, \psi_{i, n}^{h}:=\psi_{\delta_{i, n}, \xi_{i, n}}^{h}$, and $\Omega_{n}:=\Omega_{\varepsilon_{n}}$.

Let $\mathbf{h}_{n}:=\mathbf{L}_{n}\left(\boldsymbol{\phi}_{n}\right)$. Then, observing that

$$
\mu_{i} f^{\prime}\left(\mu_{i}^{-\frac{1}{p-1}} P_{\varepsilon} U_{\delta, \xi}\right)=p\left(P_{\varepsilon} U_{\delta, \xi}\right)^{p-1} \quad \forall \varepsilon, \delta>0, \xi \in \mathbb{R}^{N},
$$

we have by definition of $\mathbf{L}_{n}$

$$
\begin{aligned}
\phi_{i, n}=i^{*}[ & p\left(P_{n} U_{i, n}\right)^{p-1} \phi_{i, n}+\frac{p-1}{2} \sum_{j \neq i} \beta_{i j}\left(\mu_{j}^{-\frac{1}{p-1}} P_{n} U_{j, n}\right)^{\frac{p+1}{2}}\left(\mu_{i}^{-\frac{1}{p-1}} P_{n} U_{i, n}\right)^{\frac{p-3}{2}} \phi_{i, n} \\
& \left.+\frac{p+1}{2} \sum_{j \neq i} \beta_{i j}\left(\mu_{j}^{-\frac{1}{p-1}} P_{n} U_{j, n}\right)^{\frac{p-1}{2}}\left(\mu_{i}^{-\frac{1}{p-1}} P_{n} U_{i, n}\right)^{\frac{p-1}{2}} \phi_{j, n}\right]+h_{i, n}-w_{i, n}
\end{aligned}
$$

for some $w_{i, n} \in K_{i, n}$.

Step 1) We show that $\left\|w_{i, n}\right\|_{H_{0}^{1}\left(\Omega_{n}\right)} \rightarrow 0$ as $n \rightarrow \infty$.

Since $w_{i, n} \in K_{i, n}$, there exists constants $c_{i, n}^{k}$ such that

$$
w_{i, n}=\sum_{k=0}^{N} c_{i, n}^{k} P_{n} \psi_{i, n}^{k}
$$


Let us multiply equation (3.8) with $\delta_{i, n}^{2} w_{i, n}$ : taking into account that $\phi_{i, n}, h_{i, n} \in K_{i, n}^{\perp}$, we deduce that

$$
\begin{array}{r}
\underbrace{\delta_{i, n}^{2}\left\|w_{i, n}\right\|_{H_{0}^{1}\left(\Omega_{n}\right)}^{2}}_{=:(I)}=p \underbrace{p \underbrace{2}_{i, n} \int_{\Omega_{n}}\left(P_{n} U_{i, n}\right)^{p-1} \phi_{i, n} w_{i, n}}_{=:(I I)} \\
+\frac{p-1}{2} \sum_{l=0}^{N} c_{i, n}^{l} \underbrace{\delta_{i, n}^{2} \int_{\Omega_{n}} \sum_{j \neq i} \beta_{i j}\left(\mu_{j}^{-\frac{1}{p-1}} P_{n} U_{j, n}\right)^{\frac{p+1}{2}}\left(\mu_{i}^{-\frac{1}{p-1}} P_{n} U_{i, n}\right)^{\frac{p-3}{2}} \phi_{i, n}\left(P_{n} \psi_{i, n}^{l}\right)}_{=:(I I I)} \\
+\frac{p+1}{2} \sum_{l=0}^{N} c_{i, n}^{l} \underbrace{\delta_{i, n}^{2} \int_{\Omega_{n}} \sum_{j \neq i} \beta_{i j}\left(\mu_{j}^{-\frac{1}{p-1}} P_{n} U_{j, n}\right)^{\frac{p-1}{2}}\left(\mu_{i}^{-\frac{1}{p-1}} P_{n} U_{i, n}\right)^{\frac{p-1}{2}} \phi_{j, n}\left(P_{n} \psi_{i, n}^{l}\right)}_{=:(I V)} .
\end{array}
$$

The rest of the proof of step 1 consists in a careful (and very long) asymptotic expansion of the terms $(I)-(I V)$, whose details are contained in Appendix B. Therein we prove that

$$
\begin{aligned}
|(I)| & =\sum_{l=0}^{N}\left(c_{i, n}^{l}\right)^{2} \sigma_{l l}+o(1) \sum_{l, k=0}^{N} c_{i, n}^{l} c_{i, n}^{k} \\
|(I I)| & =o\left(\delta_{i, n}^{2}\right)\left\|w_{i, n}\right\|_{H_{0}^{1}\left(\Omega_{n}\right)}+O\left(\delta_{i, n}^{2}\right) \sum_{l=0}^{N} c_{i, n}^{l} \\
|(I I I)| & =o\left(\delta_{i, n}^{2}\right) \\
|(I V)| & =o\left(\delta_{i, n}^{2}\right),
\end{aligned}
$$

as $n \rightarrow \infty$, with

$$
\sigma_{l k}= \begin{cases}0 & \text { if } l \neq k \\ p \alpha_{N}^{p+1}(N-2)^{2} \int_{\mathbb{R}^{N}} \frac{y_{l}^{2}}{\left(1+|y|^{2}{ }^{N+2}\right.} d y & \text { if } k=l \geq 1 \\ p \alpha_{N}^{p+1}\left(\frac{N-2}{2}\right)^{2} \int_{\mathbb{R}^{N}} \frac{\left.(\mid y)^{2}-1\right)^{2}}{\left(1+|y|^{2}\right)^{N+2}} d y & \text { if } k=l=0 .\end{cases}
$$

Using the second to fourth estimates in (3.10), equation (3.9) becomes

$$
\delta_{i, n}^{2}\left\|w_{i, n}\right\|_{H_{0}^{1}\left(\Omega_{n}\right)}^{2}=o\left(\delta_{i, n}^{2}\right)\left\|w_{i, n}\right\|_{H_{0}^{1}\left(\Omega_{n}\right)}+O\left(\delta_{i, n}^{2}\right) \sum_{k=0}^{N} c_{i, n}^{k} .
$$

Due to the first estimate in (3.10), the previous expression yields

$$
C\left(\sum_{k=0}^{N}\left|c_{i, n}^{k}\right|\right)^{2} \leq o(1) \sum_{k=0}^{N}\left|c_{i, n}^{k}\right|,
$$

so that, firstly, $\left\{c_{i, n}^{k}\right\}$ is a bounded sequence, for any $k$, and in turn, this implies that $c_{i, n}^{k} \rightarrow 0$ as $n \rightarrow \infty$, for every $k$. Hence, using this into (3.12), we deduce that

$$
\left\|w_{i, n}\right\|_{H_{0}^{1}\left(\Omega_{n}\right)}^{2}=o(1)\left\|w_{i, n}\right\|_{H_{0}^{1}\left(\Omega_{n}\right)}+o(1),
$$

whence $\left\|w_{i, n}\right\|_{H_{0}^{1}\left(\Omega_{n}\right)} \rightarrow 0$ as $n \rightarrow \infty$. 
Step 2) For a fixed $\kappa=1, \ldots, m$, let us introduce

$$
\tilde{\phi}_{i, n}^{\kappa}(y):= \begin{cases}\delta_{\kappa, n}^{\frac{N-2}{2}} \phi_{i, n}\left(\xi_{\kappa, n}+\delta_{\kappa, n} y\right) & y \in \frac{\Omega_{n}-\xi_{\kappa, n}}{\delta_{\kappa, n}}=: \tilde{\Omega}_{\kappa, n} \quad i=1, \ldots, m . \\ 0 & y \in \mathbb{R}^{N} \backslash \tilde{\Omega}_{\kappa, n},\end{cases}
$$

In a completely analogue way, we define $\tilde{h}_{i, n}^{\kappa}$ and $\tilde{w}_{i, n}^{\kappa}$, and we set $\tilde{\phi}_{\kappa, n}:=\tilde{\phi}_{\kappa, n}^{\kappa}, \tilde{h}_{\kappa, n}:=\tilde{h}_{\kappa, n}^{\kappa}$, $\tilde{w}_{\kappa, n}:=\tilde{w}_{\kappa, n}^{\kappa}$.

In this step we show that $\tilde{\phi}_{\kappa, n} \rightarrow 0$ in $\mathcal{D}^{1,2}\left(\mathbb{R}^{N}\right)$ (i.e. $\nabla \tilde{\phi}_{\kappa, n} \rightarrow 0$ in $L^{2}\left(\mathbb{R}^{N}\right)$ ) as $n \rightarrow \infty$, for every $\kappa=1, \ldots, m$.

At first, we observe that $\left\|\tilde{\phi}_{\kappa, n}\right\|_{H_{0}^{1}\left(\tilde{\Omega}_{\kappa, n}\right)}=\left\|\phi_{\kappa, n}\right\|_{H_{0}^{1}\left(\Omega_{n}\right)} \leq 1$, and hence up to a subsequence $\tilde{\phi}_{\kappa, n} \rightarrow \tilde{\phi}_{\kappa}$ weakly in $\mathcal{D}^{1,2}\left(\mathbb{R}^{N}\right)$ for every $i$. Now we rewrite the equation (3.8) for $\phi_{\kappa, n}$ in terms of $\tilde{\phi}_{\kappa, n}:$ if $\psi \in \mathcal{C}_{c}^{\infty}\left(\mathbb{R}^{N}\right)$, we have

$$
\begin{aligned}
\int_{\tilde{\Omega}_{\kappa, n}} \nabla \tilde{\phi}_{\kappa, n} & \cdot \nabla \psi=p \delta_{\kappa, n}^{2} \int_{\tilde{\Omega}_{\kappa, n}}\left(\widehat{P_{n} U_{\kappa, n}}\right)^{p-1} \tilde{\phi}_{\kappa, n} \psi \\
& +\frac{p-1}{2} \sum_{j \neq \kappa} \delta_{\kappa, n}^{2} \beta_{\kappa j} \int_{\tilde{\Omega}_{\kappa, n}}\left(\mu_{\kappa}^{-\frac{1}{p-1}} \widehat{P_{n} U_{\kappa, n}}\right)^{\frac{p-3}{2}}\left(\mu_{j}^{-\frac{1}{p-1}} \widehat{P_{n} U_{j, n}}\right)^{\frac{p+1}{2}} \tilde{\phi}_{\kappa, n} \psi \\
& +\frac{p+1}{2} \sum_{j \neq \kappa} \delta_{\kappa, n}^{2} \beta_{\kappa j} \int_{\tilde{\Omega}_{\kappa, n}}\left(\mu_{\kappa}^{-\frac{1}{p+1}} \widehat{P_{n} U_{\kappa, n}}\right)^{\frac{p-1}{2}}\left(\mu_{j}^{-\frac{1}{p-1}} \widehat{P_{n} U_{j, n}}\right)^{\frac{p-1}{2}} \tilde{\phi}_{j, n} \psi \\
& +\int_{\tilde{\Omega}_{\kappa, n}} \nabla \tilde{\phi}_{\kappa, n} \cdot \nabla\left(\tilde{h}_{\kappa, n}-\tilde{w}_{\kappa, n}\right),
\end{aligned}
$$

where $\widehat{P_{n} U_{j, n}}(y):=P_{n} U_{j, n}\left(\xi_{\kappa, n}+\delta_{\kappa, n} y\right)$ for all $j=1, \ldots, m$. In Appendix C, we show that (3.13) yields

$$
\int_{\tilde{\Omega}_{\kappa, n}} \nabla \tilde{\phi}_{\kappa, n} \cdot \nabla \psi=p \int_{\mathbb{R}^{N}} U_{1,0}^{p-1} \tilde{\phi}_{\kappa} \psi+o(1) \quad \text { for every } \psi \in \mathcal{C}_{c}^{\infty}\left(\mathbb{R}^{N}\right)
$$

which in turn, by weak convergence, implies that

$$
-\Delta \tilde{\phi}_{\kappa}=p U_{1,0}^{p-1} \tilde{\phi}_{\kappa}, \quad \tilde{\phi}_{i} \in \mathcal{D}^{1,2}\left(\mathbb{R}^{N}\right)
$$

Since our final goal consists in proving that $\tilde{\phi}_{\kappa}=0$, due to the previous equation it will suffices to show that $\tilde{\phi}_{\kappa}$ is orthogonal, in $\mathcal{D}^{1,2}\left(\mathbb{R}^{N}\right)$, to the $N+1$ partial derivatives $\psi_{1,0}^{\ell}(\ell=0, \ldots, N)$ of $U_{1,0}$. Indeed, we already know that these partial derivatives span the sets of the solutions to (3.15). The orthogonality condition comes from the fact that $\phi_{\kappa, n} \in K_{\kappa, n}^{\perp}$ for every $i$ and $n$ : indeed, for any $\ell=1, \ldots, N$ (the case $\ell=0$ is analogue), we have

$$
\begin{aligned}
0 & =\delta_{\kappa, n} \int_{\Omega_{n}} \nabla \phi_{\kappa, n} \cdot \nabla\left(P_{n} \psi_{\kappa, n}^{\ell}\right)=p \delta_{\kappa, n} \int_{\Omega_{n}} p U_{\kappa, n}^{p-1} \psi_{\kappa, n}^{\ell} \phi_{\kappa, n} \\
& =\delta_{\kappa, n} \int_{\Omega_{n}} p(N-2) \alpha_{N}^{p}\left(\frac{\delta_{\kappa, n}}{\delta_{\kappa, n}^{2}+\left|x-\xi_{\kappa, n}\right|^{2}}\right)^{2} \delta_{\kappa, n}^{\frac{N-2}{2}} \frac{x_{\ell}-\xi_{\kappa, n}^{\ell}}{\left(\delta_{\kappa, n}^{2}+\left|x-\xi_{\kappa, n}\right|^{2}\right)^{\frac{N}{2}}} d x \\
& =\int_{\tilde{\Omega}_{\kappa, n}} p(N-2) \alpha_{N}^{p} \frac{1}{\left(1+|y|^{2}\right)^{2}} \frac{y_{\ell}}{\left(1+|y|^{2}\right)^{\frac{N}{2}}} \tilde{\phi}_{\kappa, n} d y \\
& =\int_{\tilde{\Omega}_{\kappa, n}} p U_{1,0}^{p-1} \psi_{1,0}^{\ell} \tilde{\phi}_{\kappa, n},
\end{aligned}
$$


and by weak convergence we deduce that for every $\ell$ and $i$

$$
0=\int_{\mathbb{R}^{N}} p U_{1,0}^{p-1} \psi_{1,0}^{\ell} \tilde{\phi}_{\kappa}=\int_{\mathbb{R}^{N}} \nabla \psi_{1,0}^{\ell} \cdot \nabla \tilde{\phi}_{\kappa},
$$

as desired.

Step 3) We prove that $\left\|\phi_{i, n}\right\|_{H_{0}^{1}\left(\Omega_{n}\right)} \rightarrow 0$ as $n \rightarrow \infty$ for every $i$. This is in contradiction with the fact that $\left\|\phi_{n}\right\|_{H_{0}^{1}\left(\Omega_{n}\right)}=1$, and completes the proof of (3.7). Let us test (3.8) with $\phi_{i, n}$ : recalling that $\left\{\phi_{i, n}\right\}$ is bounded in $H_{0}^{1}\left(\Omega_{n}\right)$ and that $w_{i, n}, h_{i, n} \rightarrow 0$ strongly, we deduce that

$$
\begin{array}{r}
\left\|\phi_{i, n}\right\|_{H_{0}^{1}\left(\Omega_{n}\right)}^{2}=o(1)+\underbrace{p \int_{\Omega_{n}}\left(P_{n} U_{i, n}\right)^{p-1} \phi_{i, n}^{2}}_{=:(I)} \\
+\frac{p-1}{2} \sum_{j \neq i} \underbrace{\beta_{i j} \int_{\Omega_{n}}\left(\mu_{i}^{-\frac{1}{p-1}} P_{n} U_{i, n}\right)^{\frac{p-3}{2}}\left(\mu_{j}^{-\frac{1}{p-1}} P_{n} U_{j, n}\right)^{\frac{p+1}{2}} \phi_{i, n}^{2}}_{=:(I I)} \\
+\frac{p+1}{2} \sum_{j \neq i} \underbrace{\beta_{i j} \int_{\Omega_{n}}^{\left.\int_{i}^{-\frac{1}{p-1}} P_{n} U_{i, n}\right)^{\frac{p-1}{2}}\left(\mu_{j}^{-\frac{1}{p-1}} P_{n} U_{j, n}\right)^{\frac{p-1}{2}} \phi_{i, n} \phi_{j, n}}}_{=:(I I I)}
\end{array}
$$

We have to estimate the right hand side. At first, recalling as usual that $0 \leq P_{n} U_{i, n} \leq U_{i, n}$, we have

$$
|(I)| \leq p \int_{\Omega_{n}} U_{i, n}^{p-1} \phi_{i, n}^{2}=C p \int_{\tilde{\Omega}_{i, n}} U_{1,0}^{p-1} \tilde{\phi}_{i, n}^{2} \rightarrow 0
$$

as $n \rightarrow \infty$, since $\tilde{\phi}_{i, n}^{2} \rightarrow 0$ in $L^{\frac{N}{N-2}}\left(\mathbb{R}^{N}\right)$ by step 2 , and $U_{1,0}^{p-1} \in L^{\frac{N}{2}}\left(\mathbb{R}^{N}\right)$.

The second term on the right hand side in (3.16) can be estimated discussing several possibilities: if $\beta_{i j}<0$, we simply observe that $(I I) \leq 0$. Otherwise, if $\beta_{i j}>0$,

$$
\begin{aligned}
(I I) & \leq C \beta_{i j} \int_{\Omega_{n}} U_{i, n}^{\frac{p-3}{2}} U_{j, n}^{\frac{p+1}{2}} \phi_{i, n}^{2} \\
& \leq C \beta_{i j}\left(\int_{\Omega_{n}} U_{i, n}^{\frac{(4-N) N}{2(N-2)}} U_{j, n}^{\frac{N^{2}}{2(N-2)}}\right)^{\frac{2}{N}}\left|\phi_{i, n}\right|_{2^{*}}^{2} .
\end{aligned}
$$

In case $N=3$, the last term reads

$$
C \beta_{i j}\left(\int_{\Omega_{n}} U_{i, n}^{\frac{3}{2}} U_{j, n}^{\frac{9}{2}}\right)^{\frac{2}{3}}\left|\phi_{i, n}\right|_{2^{*}}^{2} \leq C \beta_{i j}\left\|\phi_{i, n}\right\|_{H_{0}^{1}\left(\Omega_{n}\right)}^{2}\left(\delta_{i, n}^{\frac{3}{4}} \delta_{j, n}^{\frac{3}{4}}\right)^{\frac{2}{3}}=o(1)
$$

(no matter how large $\beta_{i j}$ is), where we used Lemmas A.6 and A.8. If on the other hand $N=4$, we have

$$
C \beta_{i j}\left(\int_{\Omega_{n}} U_{j, n}^{4}\right)^{\frac{1}{2}}\left|\phi_{i, n}\right|_{2^{*}}^{2} \leq C \beta_{i j}\left\|\phi_{i, n}\right\|_{H_{0}^{1}\left(\Omega_{n}\right)}^{2},
$$

where $C$ does not depend on $\beta_{i j}$. We conclude that there exists $\bar{\beta}>0$ sufficiently small such that, if $\beta_{i j}<\bar{\beta}$ for all $i \neq j$, then

$$
|(I I)| \leq C \beta_{i j}\left\|\phi_{i, n}\right\|_{H_{0}^{1}\left(\Omega_{n}\right)}^{2} \leq \frac{1}{2}\left\|\phi_{i, n}\right\|_{H_{0}^{1}\left(\Omega_{n}\right)}^{2}
$$


To sum up, in any case we can conclude that for every $i \neq j$ and for any $n$ large enough

$$
\frac{p-1}{2} \sum_{j \neq i}(I I) \leq \frac{1}{2}\left\|\phi_{i, n}\right\|_{H_{0}^{1}\left(\Omega_{n}\right)}^{2} \quad\left\{\begin{array}{l}
\text { for every } \beta_{i j}, \text { if } N=3 \\
\text { provided } \beta_{i j}<\bar{\beta}, \text { if } N=4 .
\end{array}\right.
$$

It remains to consider the last term in (3.16): by the Hölder inequality and Lemmas A.6 and A.8

$$
\begin{aligned}
|(I I I)| & \leq \beta_{i j} \int_{\Omega_{n}} U_{i, n}^{\frac{p-1}{2}} U_{j, n}^{\frac{p-1}{2}}\left|\phi_{i, n}\right|\left|\phi_{j, n}\right| \\
& \leq \beta_{i j}\left|\phi_{i, n}\right|_{2^{*}}\left|\phi_{j, n}\right|_{2^{*}}\left(\int_{\Omega_{n}} U_{i, n}^{\frac{N}{N-2}} U_{j, n}^{\frac{N}{N-2}}\right)^{\frac{2}{N}} \\
& \leq C \beta_{i j}\left\|\phi_{i, n}\right\|_{H_{0}^{1}\left(\Omega_{n}\right)}\left\|\phi_{j, n}\right\|_{H_{0}^{1}\left(\Omega_{n}\right)} \delta_{i, n} \delta_{j, n}\left(\left|\log \delta_{i, n}\right|+\left|\log \delta_{j, n}\right|\right)^{\frac{2}{N}}=o(1)
\end{aligned}
$$

as $n \rightarrow \infty$, where we used the boundedness of $\left\{\phi_{n}\right\}$ in $H_{0}^{1}$.

Plugging (3.17)-(3.20) into (3.16), we conclude that $\phi_{i, n} \rightarrow 0$ strongly in $H_{0}^{1}\left(\Omega_{n}\right)$, which gives the desired contradiction and completes the first part of the lemma.

It remains still to show the invertibility of $\mathbf{L}_{\mathbf{d}, \boldsymbol{\tau}, \varepsilon}$, and this is the object of the last step.

Step 4) We start recalling that the operator $i^{*}: L^{\frac{2 N}{N+2}}\left(\Omega_{\varepsilon}\right) \rightarrow H_{0}^{1}\left(\Omega_{\varepsilon}\right)$ is compact. Therefore, by definition, the restriction of $\mathbf{L}_{\mathbf{d}, \boldsymbol{\tau}, \varepsilon}$ to $\mathbf{K}_{\mathbf{d}, \boldsymbol{\tau}, \varepsilon}^{\perp}$ is a compact perturbation of the identity. So far we showed that

$$
\left\|\mathbf{L}_{\mathbf{d}, \boldsymbol{\tau}, \varepsilon}(\boldsymbol{\phi})\right\|_{H_{0}^{1}\left(\Omega_{\varepsilon}, \mathbb{R}^{m}\right)} \geq C\|\boldsymbol{\phi}\|_{H_{0}^{1}\left(\Omega_{\varepsilon}, \mathbb{R}^{m}\right)} \quad \text { for every } \boldsymbol{\phi} \in \mathbf{K}_{\mathbf{d}, \boldsymbol{\tau}, \varepsilon}^{\perp},
$$

and hence $\mathbf{L}_{\mathbf{d}, \boldsymbol{\tau}, \varepsilon}$ is injective. By the Fredholm alternative, it is also surjective, thus invertible, and the inverse is continuous (due to $(3.21)$ ).

Now we prove the solvability of equation (3.5).

Lemma 3.3. Let $N=4$. For every $\eta>0$ small enough there exists $\bar{\beta}, \varepsilon_{1}>0$ small and $C>0$ such that: if $\varepsilon \in\left(0, \varepsilon_{1}\right)$, and $-\infty<\beta_{i j}<\bar{\beta}$ for every $i \neq j$, then for any $(\mathbf{d}, \boldsymbol{\tau}) \in X_{\eta}$ there exists a unique function $\boldsymbol{\phi}^{\mathbf{d}, \boldsymbol{\tau}, \varepsilon} \in K_{\mathbf{d}, \boldsymbol{\tau}, \varepsilon}^{\perp}$ solving equation (3.5):

$$
\mathbf{L}_{\mathbf{d}, \boldsymbol{\tau}, \varepsilon}\left(\phi^{\mathbf{d}, \boldsymbol{\tau}, \varepsilon}\right)=\mathbf{R}_{\mathbf{d}, \boldsymbol{\tau}, \varepsilon}+\mathbf{N}_{\mathbf{d}, \boldsymbol{\tau}, \varepsilon}\left(\phi^{\mathbf{d}, \boldsymbol{\tau}, \varepsilon}\right),
$$

and satysfying

$$
\left\|\phi^{\mathbf{d}, \boldsymbol{\tau}, \varepsilon}\right\|_{H_{0}^{1}\left(\Omega_{\varepsilon}\right)} \leq C \varepsilon^{\frac{N-2}{2}} .
$$

If $N=3$, the same conclusion holds without any restriction on $\beta_{i j}$.

Proof. Let $\varepsilon \in\left(0, \varepsilon_{0}\right)$, with $\varepsilon_{0}$ given by Lemma 3.2. Then, to solve equation (3.5) is equivalent to find $\boldsymbol{\phi} \in \mathbf{K}_{\mathbf{d}, \boldsymbol{\tau}, \varepsilon}^{\perp}$ such that

$$
\phi=\mathbf{L}_{\mathbf{d}, \boldsymbol{\tau}, \varepsilon}^{-1}\left(\mathbf{R}_{\mathbf{d}, \boldsymbol{\tau}, \varepsilon}+\mathbf{N}_{\mathbf{d}, \boldsymbol{\tau}, \varepsilon}(\phi)\right)=: \mathbf{T}_{\mathbf{d}, \boldsymbol{\tau}, \varepsilon}(\phi) .
$$

We aim at proving that $\mathbf{T}_{\mathbf{d}, \boldsymbol{\tau}, \varepsilon}$ is a contraction inside a properly chosen region.

Step 1) $\mathbf{T}_{\mathbf{d}, \boldsymbol{\tau}, \varepsilon}: Y_{\varepsilon} \rightarrow Y_{\varepsilon}$ for a suitable subset $Y_{\varepsilon} \subset H_{0}^{1}\left(\Omega_{\varepsilon}, \mathbb{R}^{m}\right)$.

Using the continuity of $\mathbf{L}_{\mathbf{d}, \boldsymbol{\tau}, \varepsilon}^{-1}$, of $\Pi_{i}^{\perp}$ and of $i^{*}$, we have

$$
\left\|\mathbf{T}_{\mathbf{d}, \boldsymbol{\tau}, \varepsilon}(\phi)\right\|_{H_{0}^{1}\left(\Omega_{\varepsilon}\right)} \leq C\left(\left|\tilde{\mathbf{R}}_{\mathbf{d}, \boldsymbol{\tau}, \varepsilon}\right|_{L^{\frac{2 N}{N+2}}\left(\Omega_{\varepsilon}\right)}+\left|\tilde{\mathbf{N}}_{\mathbf{d}, \boldsymbol{\tau}, \varepsilon}(\phi)\right|_{L^{\frac{2 N}{N+2}}\left(\Omega_{\varepsilon}\right)}\right)
$$


where

$$
\begin{aligned}
& \tilde{R}_{\mathbf{d}, \boldsymbol{\tau}, \varepsilon}^{i}:=\mu_{i}^{-\frac{1}{p-1}}\left(P_{\varepsilon} U_{\delta_{i}, \xi_{i}}^{p}-U_{\delta_{i}, \xi_{i}}^{p}\right)+\sum_{j \neq i} \beta_{i j}\left(\mu_{j}^{-\frac{1}{p-1}} P_{\varepsilon} U_{\delta_{j}, \xi_{j}}\right)^{\frac{p+1}{2}}\left(\mu_{i}^{-\frac{1}{p-1}} P_{\varepsilon} U_{\delta_{i}, \xi_{i}}\right)^{\frac{p-1}{2}}, \\
& \tilde{N}_{\mathbf{d}, \boldsymbol{\tau}, \varepsilon}^{i}(\phi):=\tilde{P}_{\mathbf{d}, \boldsymbol{\tau}, \varepsilon}^{i}(\phi)+\tilde{Q}_{\mathbf{d}, \boldsymbol{\tau}, \varepsilon}^{i}(\phi), \\
& \tilde{P}_{\mathbf{d}, \boldsymbol{\tau}, \varepsilon}^{i}(\phi):=\mu_{i} f\left(\mu_{i}^{-\frac{1}{p-1}} P_{\varepsilon} U_{\delta_{i}, \xi_{i}}+\phi_{i}\right)-\mu_{i} f\left(\mu_{i}^{-\frac{1}{p-1}} P_{\varepsilon} U_{\delta_{i}, \xi_{i}}\right)-p\left(P_{\varepsilon} U_{\delta_{i}, \xi_{i}}\right)^{p-1} \phi_{i},
\end{aligned}
$$

and

$$
\begin{aligned}
\tilde{Q}_{\mathbf{d}, \boldsymbol{\tau}, \varepsilon}^{i}(\phi): & =\sum_{j \neq i} \beta_{i j}\left|\mu_{j}^{-\frac{1}{p-1}} P_{\varepsilon} U_{\delta_{j}, \xi_{j}}+\phi_{j}\right|^{\frac{p+1}{2}}\left|\mu_{i}^{-\frac{1}{p-1}} P_{\varepsilon} U_{\delta_{i}, \xi_{i}}+\phi_{i}\right|^{\frac{p-3}{2}}\left(\mu_{i}^{-\frac{1}{p-1}} P_{\varepsilon} U_{\delta_{i}, \xi_{i}}+\phi_{i}\right) \\
& -\sum_{j \neq i} \beta_{i j}\left(\mu_{j}^{-\frac{1}{p-1}} P_{\varepsilon} U_{\delta_{j}, \xi_{j}}\right)^{\frac{p+1}{2}}\left(\mu_{i}^{-\frac{1}{p-1}} P_{\varepsilon} U_{\delta_{i}, \xi_{i}}\right)^{\frac{p-1}{2}} \\
& -\frac{p-1}{2} \sum_{j \neq i} \beta_{i j}\left(\mu_{j}^{-\frac{1}{p-1}} P_{\varepsilon} U_{\delta_{j}, \xi_{j}}\right)^{\frac{p+1}{2}}\left(\mu_{i}^{-\frac{1}{p-1}} P_{\varepsilon} U_{\delta_{i}, \xi_{i}}\right)^{\frac{p-3}{2}} \phi_{i} \\
& -\frac{p+1}{2} \sum_{j \neq i} \beta_{i j}\left(\mu_{j}^{-\frac{1}{p-1}} P_{\varepsilon} U_{\delta_{j}, \xi_{j}}\right)^{\frac{p-1}{2}}\left(\mu_{i}^{-\frac{1}{p-1}} P_{\varepsilon} U_{\delta_{i}, \xi_{i}}\right)^{\frac{p-1}{2}} \phi_{j},
\end{aligned}
$$

with $i=1, \ldots, m$. In Appendix $\mathrm{D}$, we prove that

$$
\begin{aligned}
& \left|\tilde{R}_{\mathbf{d}, \boldsymbol{\tau}, \varepsilon}^{i}\right|_{L^{\frac{2 N}{N+2}}\left(\Omega_{\varepsilon}\right)} \leq C \varepsilon^{\frac{N-2}{2}} \\
& \left|\tilde{N}_{\mathbf{d}, \boldsymbol{\tau}, \varepsilon}^{i}(\phi)\right|_{L^{\frac{2 N}{N+2}}\left(\Omega_{\varepsilon}\right)} \leq C\|\phi\|_{H_{0}^{1}\left(\Omega_{\varepsilon}\right)}^{2}
\end{aligned}
$$

Therefore, by equation (3.22) there exist $C_{1}, C_{2}>0$ such that

$$
\left\|\mathbf{T}_{\mathbf{d}, \boldsymbol{\tau}, \varepsilon}(\phi)\right\|_{H_{0}^{1}\left(\Omega_{\varepsilon}\right)} \leq C_{1} \varepsilon^{\frac{N-2}{2}}+C_{2}\|\phi\|_{H_{0}^{1}\left(\Omega_{\varepsilon}\right)}^{2}
$$

Let $\bar{C}>C_{1}$ arbitrarily chosen, and let

$$
Y_{\varepsilon}:=\left\{\phi \in H_{0}^{1}\left(\Omega_{\varepsilon}, \mathbb{R}^{m}\right):\|\phi\|_{H_{0}^{1}\left(\Omega_{\varepsilon}\right)} \leq \bar{C} \varepsilon^{\frac{N-2}{2}}\right\} .
$$

Then there exists $\varepsilon_{1} \in\left(0, \varepsilon_{0}\right]$ sufficiently small such that

$$
\left\|\mathbf{T}_{\mathbf{d}, \boldsymbol{\tau}, \varepsilon}(\phi)\right\|_{H_{0}^{1}\left(\Omega_{\varepsilon}\right)} \leq C_{1} \varepsilon^{\frac{N-2}{2}}+C_{2} \bar{C}^{2} \varepsilon^{N-2} \leq \bar{C} \varepsilon^{\frac{N-2}{2}}
$$

for every $\varepsilon \in\left(0, \varepsilon_{1}\right)$ and $\phi \in H_{0}^{1}\left(\Omega_{\varepsilon}, \mathbb{R}^{m}\right)$, that is, $\mathbf{T}_{\mathbf{d}, \boldsymbol{\tau}, \varepsilon}: Y_{\varepsilon} \rightarrow Y_{\varepsilon}$.

Step 2) $\mathbf{T}_{\mathbf{d}, \boldsymbol{\tau}, \varepsilon}$ is a contraction in $Y_{\varepsilon}$.

Notice that

$$
\left\|\mathbf{T}_{\mathbf{d}, \boldsymbol{\tau}, \varepsilon}\left(\phi^{1}\right)-\mathbf{T}_{\mathbf{d}, \boldsymbol{\tau}, \varepsilon}\left(\phi^{2}\right)\right\|_{H_{0}^{1}\left(\Omega_{\varepsilon}\right)} \leq C\left|\tilde{\mathbf{N}}_{\mathbf{d}, \boldsymbol{\tau}, \varepsilon}\left(\phi^{1}\right)-\tilde{\mathbf{N}}_{\mathbf{d}, \boldsymbol{\tau}, \varepsilon}\left(\phi^{2}\right)\right|_{L^{\frac{2 N}{N+2}}\left(\Omega_{\varepsilon}\right)} .
$$

Recalling that $\tilde{N}_{\mathbf{d}, \boldsymbol{\tau}, \varepsilon}^{i}(\phi)=\tilde{P}_{\mathbf{d}, \boldsymbol{\tau}, \varepsilon}^{i}(\phi)+\tilde{Q}_{\mathbf{d}, \boldsymbol{\tau}, \varepsilon}^{i}(\phi)$, we compute with a Taylor expansion (see Lemma A.4)

$$
\left|\tilde{P}_{\mathbf{d}, \boldsymbol{\tau}, \varepsilon}^{i}\left(\phi^{1}\right)-\tilde{P}_{\mathbf{d}, \boldsymbol{\tau}, \varepsilon}^{i}\left(\phi^{2}\right)\right|_{L^{\frac{2 N}{N+2}}\left(\Omega_{\varepsilon}\right)} \leq C\left|\left(P_{\varepsilon} U_{\delta_{i}, \xi_{i}}+\left|\phi_{i}^{1}\right|+\left|\phi_{i}^{2}\right|\right)^{p-2}\left(\left|\phi_{i}^{1}\right|+\left|\phi_{i}^{2}\right|\right)\right| \phi_{i}^{1}-\phi_{i}^{2}||_{L^{\frac{2 N}{N+2}}\left(\Omega_{\varepsilon}\right)} \text {. }
$$

Therefore, by the Hölder and the Sobolev inequalities

$$
\begin{aligned}
\left|\tilde{P}_{\mathbf{d}, \boldsymbol{\tau}, \varepsilon}^{i}\left(\phi^{1}\right)-\tilde{P}_{\mathbf{d}, \boldsymbol{\tau}, \varepsilon}^{i}\left(\phi^{2}\right)\right|_{L^{\frac{2 N}{N+2}}\left(\Omega_{\varepsilon}\right)} & \leq\left(C+\left|\phi_{i}^{1}\right|_{2^{*}}^{p-2}+\left|\phi_{i}^{2}\right|_{2^{*}}^{p-2}\right)\left(\left|\phi_{i}^{1}\right|_{2^{*}}+\left|\phi_{i}^{2}\right|_{2^{*}}\right)\left|\phi_{i}^{1}-\phi_{i}^{2}\right|_{2^{*}} \\
& \leq C\|\phi\|_{H_{0}^{1}\left(\Omega_{\varepsilon}\right)}\left\|\phi_{i}^{1}-\phi_{i}^{2}\right\|_{H_{0}^{1}\left(\Omega_{\varepsilon}\right)} \leq C \varepsilon^{\frac{N-2}{2}}\left\|\phi_{i}^{1}-\phi_{i}^{2}\right\|_{H_{0}^{1}\left(\Omega_{\varepsilon}\right)}
\end{aligned}
$$


for every $\phi^{1}, \phi^{2} \in Y_{\varepsilon}, i=1, \ldots, m$. Regarding $\tilde{Q}_{\boldsymbol{\delta}, \boldsymbol{\tau}, \varepsilon}^{i}$, in Appendix D we show that

$$
\left|\tilde{Q}_{\mathbf{d}, \boldsymbol{\tau}, \varepsilon}^{i}\left(\phi^{1}\right)-\tilde{Q}_{\mathbf{d}, \boldsymbol{\tau}, \varepsilon}^{i}\left(\phi^{2}\right)\right|_{L^{\frac{2 N}{N+2}}\left(\Omega_{\varepsilon}\right)} \leq C \varepsilon^{\frac{N-2}{2}}\left\|\phi^{1}-\phi^{2}\right\|_{H_{0}^{1}\left(\Omega_{\varepsilon}\right)}
$$

or every $\phi^{1}, \phi^{2} \in Y_{\varepsilon}, i=1, \ldots, m$. Collecting together (3.24), (3.25) and (3.26), we deduce that there exists $C>0$ such that

$$
\left\|\mathbf{T}_{\mathbf{d}, \boldsymbol{\tau}, \varepsilon}\left(\boldsymbol{\phi}^{1}\right)-\mathbf{T}_{\mathbf{d}, \boldsymbol{\tau}, \varepsilon}\left(\phi^{2}\right)\right\|_{H_{0}^{1}\left(\Omega_{\varepsilon}\right)} \leq C \varepsilon^{\frac{N-2}{2}}\left\|\phi^{1}-\phi^{2}\right\|_{H_{0}^{1}\left(\Omega_{\varepsilon}\right)} \quad \forall \phi^{1}, \phi^{2} \in Y_{\varepsilon} .
$$

By replacing the necessary $\varepsilon_{1}$ with a smaller quantity, we see that for $\varepsilon \in\left(0, \varepsilon_{1}\right)$ the map $\mathbf{T}_{\mathbf{d}, \boldsymbol{\tau}, \varepsilon}$ is a contraction in $Y_{\varepsilon}$, and hence the thesis follows by the contraction mapping theorem.

Lemma 3.3 enables us to define a map

$$
A:\left(0, \varepsilon_{1}\right) \times X_{\eta} \rightarrow \mathbf{K}_{\mathbf{d}, \boldsymbol{\tau}, \varepsilon}^{\perp}, \quad(\varepsilon, \mathbf{d}, \boldsymbol{\tau}) \mapsto \phi^{\mathbf{d}, \boldsymbol{\tau}, \varepsilon} .
$$

To complete the proof of Proposition 3.1, it remains to check that this map if differentiable, and to prove the desired estimate on the derivative.

Lemma 3.4. There exists $\varepsilon_{2}>0$ small enough such that the map $A$ is of class $\mathcal{C}^{1}$ in $\left(0, \varepsilon_{2}\right) \times X_{\eta}$.

Proof. We apply the implicit function theorem to $\mathbf{T}:\left(0, \varepsilon_{1}\right) \times X_{\eta} \times \mathbf{K}_{\mathbf{d}, \boldsymbol{\tau}, \varepsilon}^{\perp} \rightarrow \mathbf{K}_{\mathbf{d}, \boldsymbol{\tau}, \varepsilon}^{\perp}$ defined by

$$
\mathbf{T}(\varepsilon, \mathbf{d}, \boldsymbol{\tau}, \boldsymbol{\phi})=\mathbf{L}_{\mathbf{d}, \boldsymbol{\tau}, \varepsilon}(\phi)-\mathbf{R}_{\mathbf{d}, \boldsymbol{\tau}, \varepsilon}-\mathbf{N}_{\mathbf{d}, \boldsymbol{\tau}, \varepsilon}(\phi) .
$$

By Lemma 3.3, we know that $\mathbf{T}\left(\varepsilon, \mathbf{d}, \boldsymbol{\tau}, \boldsymbol{\phi}^{\mathbf{d}, \boldsymbol{\tau}, \varepsilon}\right)=0$. We shall prove that $D_{\boldsymbol{\phi}} \mathbf{T}\left(\varepsilon, \mathbf{d}, \boldsymbol{\tau}, \boldsymbol{\phi}^{\mathbf{d}, \boldsymbol{\tau}, \varepsilon}\right)$ is invertible. To this aim, by the Fredholm alternative, it is sufficient to check that $D_{\phi} \mathbf{T}\left(\varepsilon, \mathbf{d}, \boldsymbol{\tau}, \boldsymbol{\phi}^{\mathbf{d}, \boldsymbol{\tau}, \varepsilon}\right)$ is injective, since $D_{\phi} \mathbf{T}\left(\varepsilon, \mathbf{d}, \boldsymbol{\tau}, \phi^{\mathbf{d}, \boldsymbol{\tau}, \varepsilon}\right)$ is a compact perturbation of the identity (due to the compactness of $\left.i^{*}\right)$.

In the rest of the proof we often write $P, U_{i}, \phi_{i}$ instead of $P_{\varepsilon}, U_{\delta_{i}, \xi_{i}}, \phi_{i}^{\mathbf{d}, \boldsymbol{\tau}, \varepsilon}$, to ease the notation.

Notice that

$$
D_{\phi} \mathbf{T}\left(\varepsilon, \mathbf{d}, \boldsymbol{\tau}, \phi^{\mathbf{d}, \boldsymbol{\tau}, \varepsilon}\right)[\boldsymbol{\psi}]=\mathbf{L}_{\mathbf{d}, \boldsymbol{\tau}, \varepsilon}(\boldsymbol{\psi})-D_{\phi} \mathbf{N}_{\mathbf{d}, \boldsymbol{\tau}, \varepsilon}\left(\phi^{\mathbf{d}, \boldsymbol{\tau}, \varepsilon}\right)[\psi] .
$$

By definition of $\mathbf{N}_{\mathbf{d}, \boldsymbol{\tau}, \varepsilon}($ see (3.3))

$$
\begin{gathered}
D_{\boldsymbol{\phi}} N_{\mathbf{d}, \boldsymbol{\tau}, \varepsilon}^{i}\left(\phi^{\mathbf{d}, \boldsymbol{\tau}, \varepsilon}\right)[\boldsymbol{\psi}]=\Pi_{i}^{\perp} \circ i^{*}\left[\mu_{i}\left(f^{\prime}\left(\mu_{i}^{-\frac{1}{p-1}} P U_{i}+\phi_{i}\right)-f^{\prime}\left(\mu_{i}^{-\frac{1}{p-1}} P U_{i}\right)\right) \psi_{i}\right. \\
+\frac{p+1}{2} \sum_{j \neq i} \beta_{i j}\left(\left|\mu_{i}^{-\frac{1}{p-1}} P U_{i}+\phi_{i}\right|^{\frac{p-3}{2}}\left|\mu_{j}^{-\frac{1}{p-1}} P U_{j}+\phi_{j}\right|^{\frac{p-3}{2}}\left(\mu_{i}^{-\frac{1}{p-1}} P U_{i}+\phi_{i}\right)\left(\mu_{j}^{-\frac{1}{p-1}} P U_{j}+\phi_{j}\right)\right. \\
\left.\quad-\left(\mu_{i}^{-\frac{1}{p-1}} P U_{i}\right)^{\frac{p-1}{2}}\left(\mu_{j}^{-\frac{1}{p-1}} P U_{j}\right)^{\frac{p-1}{2}}\right) \psi_{j} \\
+\frac{p-1}{2} \sum_{j \neq i} \beta_{i j}\left(\left|\mu_{i}^{-\frac{1}{p-1}} P U_{i}+\phi_{i}\right|^{\frac{p-3}{2}}\left|\mu_{j}^{-\frac{1}{p-1}} P U_{j}+\phi_{j}\right|^{\frac{p+1}{2}}\right. \\
\left.\left.-\left(\mu_{i}^{-\frac{1}{p-1}} P U_{i}\right)^{\frac{p-3}{2}}\left(\mu_{j}^{-\frac{1}{p-1}} P U_{j}\right)^{\frac{p+1}{2}}\right) \psi_{i}\right]
\end{gathered}
$$


Then, if $N=3$ (i.e. $p=5$ ), by the Lagrange theorem and using the fact that $0 \leq P_{\varepsilon} U_{\delta_{i}, \xi_{i}} \leq U_{\delta_{i}, \xi_{i}}$,

$$
\begin{gathered}
\left\|D_{\phi} N_{\mathbf{d}, \boldsymbol{\tau}, \varepsilon}^{i}\left(\phi^{\mathbf{d}, \boldsymbol{\tau}, \varepsilon}\right)[\boldsymbol{\psi}]\right\|_{H_{0}^{1}\left(\Omega_{\varepsilon}\right)} \leq C\left[\left|U_{i}^{3} \phi_{i} \psi_{i}\right|_{\frac{6}{5}}+\left.\left.|| \phi_{i}\right|^{4} \psi_{i}\right|_{\frac{6}{5}}\right. \\
+\sum_{j \neq i}\left(|| U_{i}+\left|\phi_{i}\right||| U_{j}+\left.\left|\phi_{j} \|^{2}\right| \phi_{i}|| \psi_{j}\right|_{\frac{6}{5}}+|| U_{i}+\left.\left|\phi_{i}\right|^{2}\left|U_{j}+\right| \phi_{j}||\left|\phi_{j}\right|\left|\psi_{j}\right|\right|_{\frac{6}{5}}\right. \\
\quad+|| \phi_{i}|| U_{j}+\left|\phi_{j}\left\|^{3}\left|\psi_{i}\right|_{\frac{6}{5}}+|| U_{i}+\left|\phi_{i}\right||| U_{j}+\left|\phi_{j} \|^{2}\right| \phi_{j}|| \psi_{i}||_{\frac{6}{5}}\right)\right]
\end{gathered}
$$

similarly, if $N=4$, using the fact that $p-3=0$, we find

$$
\begin{aligned}
& \left\|D_{\phi} N_{\mathbf{d}, \boldsymbol{\tau}, \varepsilon}^{i}\left(\phi^{\mathbf{d}, \boldsymbol{\tau}, \varepsilon}\right)[\boldsymbol{\psi}]\right\|_{H_{0}^{1}\left(\Omega_{\varepsilon}\right)} \leq C\left[\left|U_{i} \phi_{i} \psi_{i}\right|_{\frac{4}{3}}+\left.\left.|| \phi_{i}\right|^{2} \psi_{i}\right|_{\frac{4}{3}}\right. \\
& \left.\quad+\sum_{j \neq i}\left(\left|U_{i} \phi_{j} \psi_{j}\right|_{\frac{4}{3}}+\left|\phi_{i} U_{j} \psi_{j}\right|_{\frac{4}{3}}+\left|\phi_{i} \phi_{j} \psi_{j}\right|_{\frac{4}{3}}+\left|U_{j} \phi_{j} \psi_{i}\right|_{\frac{4}{3}}+\left|\phi_{j}^{2} \psi_{i}\right|_{\frac{4}{3}}\right)\right] .
\end{aligned}
$$

In order to estimate the right hand side, it is not difficult to apply the Hölder and the Sobolev inequalities, as well as the estimate in Lemma 3.3, to deduce that

$$
\left\|D_{\phi} N_{\mathbf{d}, \boldsymbol{\tau}, \varepsilon}^{i}\left(\phi^{\mathbf{d}, \boldsymbol{\tau}, \varepsilon}\right)[\boldsymbol{\psi}]\right\|_{H_{0}^{1}\left(\Omega_{\varepsilon}\right)}=o(1)\|\boldsymbol{\psi}\|_{H_{0}^{1}\left(\Omega_{\varepsilon}\right)}
$$

where $o(1) \rightarrow 0$ as $\varepsilon \rightarrow 0$ (in particular, we use the fact that $\left|U_{i}\right|_{2^{*}} \leq C$, and $\left\|\phi_{i}\right\| \rightarrow 0$ as $\varepsilon \rightarrow 0$ ).

Using (3.27) and (3.7), we infer that if $D_{\boldsymbol{\phi}} \mathbf{T}\left(\varepsilon, \mathbf{d}, \boldsymbol{\tau}, \boldsymbol{\phi}^{\mathbf{d}, \boldsymbol{\tau}, \varepsilon}\right)[\boldsymbol{\psi}]=0$, that is

$$
\mathbf{L}_{\mathbf{d}, \boldsymbol{\tau}, \varepsilon}(\boldsymbol{\psi})=D_{\phi} \mathbf{N}_{\mathbf{d}, \boldsymbol{\tau}, \varepsilon}\left(\phi^{\mathbf{d}, \boldsymbol{\tau}, \varepsilon}\right)[\boldsymbol{\psi}],
$$

then

$$
C\|\boldsymbol{\psi}\|_{H_{0}^{1}\left(\Omega_{\varepsilon}\right)} \leq o(1)\|\boldsymbol{\psi}\|_{H_{0}^{1}\left(\Omega_{\varepsilon}\right)},
$$

which finally implies that $\boldsymbol{\psi}=0$. This means that $D_{\phi} \mathbf{T}\left(\varepsilon, \mathbf{d}, \boldsymbol{\tau}, \boldsymbol{\phi}^{\mathbf{d}, \boldsymbol{\tau}, \varepsilon}\right)$ is injective for $\varepsilon$ small enough and, as observed, this suffices to complete the proof.

The following lemma completes the proof of Proposition 3.1.

Lemma 3.5. There exists $\bar{\varepsilon}>0$ small enough and a constant $C>0$ such that

$$
\left\|\nabla_{(\boldsymbol{\delta}, \boldsymbol{\xi})} \phi^{\mathbf{d}, \boldsymbol{\tau}, \varepsilon}\right\|_{H_{0}^{1}\left(\Omega_{\varepsilon}\right)} \leq C \varepsilon^{\frac{N-3}{2}} \Longleftrightarrow\left\|\nabla_{(\mathbf{d}, \boldsymbol{\tau})} \phi^{\mathbf{d}, \boldsymbol{\tau}, \varepsilon}\right\|_{H_{0}^{1}\left(\Omega_{\varepsilon}\right)} \leq C \varepsilon^{\frac{N-2}{2}}
$$

for every $\varepsilon \in(0, \bar{\varepsilon})$ and $(\mathbf{d}, \boldsymbol{\tau}) \in X_{\eta}$.

Proof. The equivalence of the two inequalities follows by the chain rule and by the ansatz (2.5). Let $s_{i, h}=\delta_{i}$ if $h=0$, and $s_{i, h}=\xi_{i, h}$ if $h=1, \ldots, N$, with $i=1, \ldots, m$. We differentiate the equation

$$
\mathbf{L}_{\mathbf{d}, \boldsymbol{\tau}, \varepsilon}\left(\phi^{\mathbf{d}, \boldsymbol{\tau}, \varepsilon}\right)=\mathbf{R}_{\mathbf{d}, \boldsymbol{\tau}, \varepsilon}-\mathbf{N}_{\mathbf{d}, \boldsymbol{\tau}, \varepsilon}\left(\phi^{\mathbf{d}, \boldsymbol{\tau}, \varepsilon}\right),
$$

with respect to a variable $s_{i, h}$, and we obtain

$$
\begin{aligned}
\partial_{s_{i, h}} \mathbf{L}_{\mathbf{d}, \boldsymbol{\tau}, \varepsilon}\left(\phi^{\mathbf{d}, \boldsymbol{\tau}, \varepsilon}\right)+\mathbf{L}_{\mathbf{d}, \boldsymbol{\tau}, \varepsilon}\left(\partial_{s_{i, h}} \boldsymbol{\phi}^{\mathbf{d}, \boldsymbol{\tau}, \varepsilon}\right) & \\
& =\partial_{s_{i, h}} \mathbf{R}_{\mathbf{d}, \boldsymbol{\tau}, \varepsilon}-\partial_{s_{i, h}} \mathbf{N}_{\mathbf{d}, \boldsymbol{\tau}, \varepsilon}\left(\phi^{\mathbf{d}, \boldsymbol{\tau}, \varepsilon}\right)-D_{\phi} \mathbf{N}_{\mathbf{d}, \boldsymbol{\tau}, \varepsilon}\left(\phi^{\mathbf{d}, \boldsymbol{\tau}, \varepsilon}\right)\left[\partial_{s_{i, h}} \boldsymbol{\phi}^{\mathbf{d}, \boldsymbol{\tau}, \varepsilon}\right] .
\end{aligned}
$$

We claim that

(3.29) $\left\|\partial_{s_{i, h}} \mathbf{L}_{\mathbf{d}, \boldsymbol{\tau}, \varepsilon}\left(\phi^{\mathbf{d}, \boldsymbol{\tau}, \varepsilon}\right)\right\|_{H_{0}^{1}\left(\Omega_{\varepsilon}\right)}+\left\|\partial_{s_{i, h}} \mathbf{R}_{\mathbf{d}, \boldsymbol{\tau}, \varepsilon}\right\|_{H_{0}^{1}\left(\Omega_{\varepsilon}\right)}+\left\|\partial_{s_{i, h}} \mathbf{N}_{\mathbf{d}, \boldsymbol{\tau}, \varepsilon}\left(\phi^{\mathbf{d}, \boldsymbol{\tau}, \varepsilon}\right)\right\|_{H_{0}^{1}\left(\Omega_{\varepsilon}\right)} \leq C \varepsilon^{\frac{N-3}{2}}$. 
With this estimate in our hands, the thesis can be easily proved. Indeed, by (3.27) and the inequality in Lemma 3.2, we deduce that

$$
\begin{aligned}
C\left\|\partial_{s_{i, h}} \phi^{\mathbf{d}, \boldsymbol{\tau}, \varepsilon}\right\| & \leq\left\|\mathbf{L}_{\mathbf{d}, \boldsymbol{\tau}, \varepsilon}\left(\partial_{s_{i, h}} \phi^{\mathbf{d}, \boldsymbol{\tau}, \varepsilon}\right)-D_{\phi} \mathbf{N}_{\mathbf{d}, \boldsymbol{\tau}, \varepsilon}\left(\phi^{\mathbf{d}, \boldsymbol{\tau}, \varepsilon}\right)\left[\partial_{s_{i, h}} \phi^{\mathbf{d}, \boldsymbol{\tau}, \varepsilon}\right]\right\| \\
& \leq\left\|\partial_{s_{i, h}} \mathbf{L}_{\mathbf{d}, \boldsymbol{\tau}, \varepsilon}\left(\phi^{\mathbf{d}, \boldsymbol{\tau}, \varepsilon}\right)\right\|+\left\|\partial_{s_{i, h}} \mathbf{R}_{\mathbf{d}, \boldsymbol{\tau}, \varepsilon}\right\|+\left\|\partial_{s_{i, h}} \mathbf{N}_{\mathbf{d}, \boldsymbol{\tau}, \varepsilon}\left(\phi^{\mathbf{d}, \boldsymbol{\tau}, \varepsilon}\right)\right\| \leq C \varepsilon^{\frac{N-3}{2}} .
\end{aligned}
$$

The validity of (3.29) can be checked by direct computations, and the details are presented in Appendix E.

\section{The REDUCED PROBLEM}

In this section we solve equation (2.7) with $\phi=\phi^{\mathbf{d}, \boldsymbol{\tau}, \varepsilon}$.

Let $J_{\varepsilon}: H_{0}^{1}\left(\Omega_{\varepsilon}, \mathbb{R}^{m}\right) \rightarrow \mathbb{R}$ be defined by

$$
J_{\varepsilon}\left(u_{1}, \ldots, u_{m}\right)=\int_{\Omega_{\varepsilon}} \frac{1}{2} \sum_{i=1}^{m}\left|\nabla u_{i}\right|^{2}-\mu_{i} F\left(u_{i}\right)-\frac{2}{p+1} \sum_{1 \leq i<j \leq m} \beta_{i j}\left|u_{i}\right|^{\frac{p+1}{2}}\left|u_{j}\right|^{\frac{p+1}{2}},
$$

where $F: \mathbb{R} \rightarrow \mathbb{R}, t \mapsto\left(t^{+}\right)^{p+1} /(p+1)$ is the primitive of $f$. Critical points of $J_{\varepsilon}$ are solution to (2.1), and hence solutions to (1.4) (here we use the fact that $\beta_{i j}=\beta_{j i}$ ).

Let us introduce the reduced functional $\tilde{J}_{\varepsilon}: X_{\eta} \rightarrow \mathbb{R}$,

$$
\tilde{J}_{\varepsilon}(\mathbf{d}, \boldsymbol{\tau}):=J_{\varepsilon}\left(\mu_{1}^{-\frac{1}{p-1}} P_{\varepsilon} U_{\delta_{1}, \xi_{1}}+\phi_{1}^{\mathbf{d}, \boldsymbol{\tau}, \varepsilon}, \ldots, \mu_{m}^{-\frac{1}{p-1}} P_{\varepsilon} U_{\delta_{m}, \xi_{m}}+\phi_{m}^{\mathbf{d}, \boldsymbol{\tau}, \varepsilon}\right) .
$$

In order to simplify the notation, from now on we often write

$$
V_{i}^{\mathbf{d}, \boldsymbol{\tau}, \varepsilon}:=\mu_{i}^{-\frac{1}{p-1}} P_{\varepsilon} U_{\delta_{i}, \xi_{i}}+\phi_{i}^{\mathbf{d}, \boldsymbol{\tau}, \varepsilon} .
$$

Lemma 4.1. There exists $\bar{\varepsilon}>0$ sufficiently small such that if $(\mathbf{d}, \boldsymbol{\tau})$ is a critical point of $\tilde{J}_{\varepsilon}$, and $\varepsilon \in(0, \bar{\varepsilon})$, then

$$
\left(\mu_{1}^{-\frac{1}{p-1}} P_{\varepsilon} U_{\delta_{1}, \xi_{1}}+\phi_{1}^{\mathbf{d}, \boldsymbol{\tau}, \varepsilon}, \ldots, \mu_{m}^{-\frac{1}{p-1}} P_{\varepsilon} U_{\delta_{m}, \xi_{m}}+\phi_{m}^{\mathbf{d}, \boldsymbol{\tau}, \varepsilon}\right)
$$

is a solution to (2.7), and hence a solution to (2.1).

Proof. We start observing that, denoting by $\langle\cdot, \cdot\rangle$ the scalar product in $H_{0}^{1}\left(\Omega_{\varepsilon}\right)$, we have

$$
\begin{aligned}
& \frac{\partial \tilde{J}_{\varepsilon}}{\partial d_{i}}(\mathbf{d}, \boldsymbol{\tau})=\sqrt{\varepsilon} \frac{\partial \tilde{J}_{\varepsilon}}{\partial \delta_{i}}(\mathbf{d}, \boldsymbol{\tau}) \\
& \quad=\sqrt{\varepsilon} d J_{\varepsilon}\left(V_{1}^{\mathbf{d}, \boldsymbol{\tau}, \varepsilon}, \ldots, V_{m}^{\mathbf{d}, \boldsymbol{\tau}, \varepsilon}\right)\left[\left(\frac{\partial \phi_{1}^{\mathbf{d}, \boldsymbol{\tau}, \varepsilon}}{\partial \delta_{i}}, \ldots, \frac{\partial \phi_{m}^{\mathbf{d}, \boldsymbol{\tau}, \varepsilon}}{\partial \delta_{i}}\right)\right] \\
& \quad+\sqrt{\varepsilon} \partial_{i} J_{\varepsilon}\left(V_{1}^{\mathbf{d}, \boldsymbol{\tau}, \varepsilon}, \ldots, V_{m}^{\mathbf{d}, \boldsymbol{\tau}, \varepsilon}\right)\left[\mu_{i}^{-\frac{1}{p-1}} P_{\varepsilon} \psi_{\delta_{i}, \xi_{i}}^{0}\right] \\
& \quad=\sqrt{\varepsilon} \sum_{k=1}^{m}\left\langle V_{k}^{\mathbf{d}, \boldsymbol{\tau}, \varepsilon}-i^{*}\left[\mu_{k} f\left(V_{k}^{\mathbf{d}, \boldsymbol{\tau}, \varepsilon}\right)+\sum_{j \neq k} \beta_{k j}\left|V_{j}^{\mathbf{d}, \boldsymbol{\tau}, \varepsilon}\right|^{\frac{p+1}{2}}\left|V_{k}^{\mathbf{d}, \boldsymbol{\tau}, \varepsilon}\right|^{\frac{p-3}{2}} V_{k}^{\mathbf{d}, \boldsymbol{\tau}, \varepsilon}\right], \frac{\partial \phi_{k}^{\mathbf{d}, \boldsymbol{\tau}, \varepsilon}}{\partial \delta_{i}}\right\rangle \\
& \quad+\sqrt{\varepsilon}\left\langle V_{i}^{\mathbf{d}, \boldsymbol{\tau}, \varepsilon}-i^{*}\left[\mu_{i} f\left(V_{i}^{\mathbf{d}, \boldsymbol{\tau}, \varepsilon}\right)+\sum_{j \neq i} \beta_{i j}\left|V_{j}^{\mathbf{d}, \boldsymbol{\tau}, \varepsilon}\right|^{\frac{p+1}{2}}\left|V_{i}^{\mathbf{d}, \boldsymbol{\tau}, \varepsilon}\right|^{\frac{p-3}{2}} V_{i}^{\mathbf{d}, \boldsymbol{\tau}, \varepsilon}\right], \mu_{i}^{-\frac{1}{p-1}} P_{\varepsilon} \psi_{\delta_{i}, \xi_{i}}^{0}\right\rangle
\end{aligned}
$$

By Proposition 3.1, we know that the projection of $\nabla J_{\varepsilon}\left(V_{1}^{\mathbf{d}, \boldsymbol{\tau}, \varepsilon}, \ldots, V_{m}^{\mathbf{d}, \boldsymbol{\tau}, \varepsilon}\right)$ on $\boldsymbol{K}_{\mathbf{d}, \boldsymbol{\tau}, \varepsilon}^{\perp}$ is 0 . This means that the terms in left position inside the brackets are linear combination of the partial 
derivatives $P_{\varepsilon} \psi_{\delta_{k}, \xi_{k}}^{\ell}$, and hence the previous chain of equalities can be continued in the following way:

$$
\frac{\partial \tilde{J}_{\varepsilon}}{\partial d_{i}}(\mathbf{d}, \boldsymbol{\tau})=\sqrt{\varepsilon} \sum_{k=1}^{m}\left\langle\sum_{\ell=0}^{N} c_{k, \ell}^{\varepsilon} P_{\varepsilon} \psi_{\delta_{k}, \xi_{k}}^{\ell}, \frac{\partial \phi_{k}^{\mathbf{d}, \boldsymbol{\tau}, \varepsilon}}{\partial \delta_{i}}\right\rangle+\sqrt{\varepsilon}\left\langle\sum_{\ell=0}^{N} c_{i, \ell}^{\varepsilon} P_{\varepsilon} \psi_{\delta_{k}, \xi_{k}}^{\ell}, \mu_{i}^{-\frac{1}{p-1}} P_{\varepsilon} \psi_{\delta_{i}, \xi_{i}}^{0}\right\rangle .
$$

Now, let $(\mathbf{d}, \boldsymbol{\tau})$ be a critical point for $\tilde{J}_{\varepsilon}$. We have then (multiplying by $\sqrt{\varepsilon}$ )

$$
\varepsilon \sum_{k=1}^{m}\left\langle\sum_{\ell=0}^{N} c_{k, \ell}^{\varepsilon} P_{\varepsilon} \psi_{\delta_{k}, \xi_{k}}^{\ell}, \frac{\partial \phi_{k}^{\mathbf{d}, \boldsymbol{\tau}, \varepsilon}}{\partial \delta_{i}}\right\rangle+\varepsilon\left\langle\sum_{\ell=0}^{N} c_{i, \ell}^{\varepsilon} P_{\varepsilon} \psi_{\delta_{k}, \xi_{k}}^{\ell}, \mu_{i}^{-\frac{1}{p-1}} P_{\varepsilon} \psi_{\delta_{i}, \xi_{i}}^{0}\right\rangle=0
$$

In the same way, if we compute the derivatives with respect to $\tau_{i, h}(h=1, \ldots, m)$ and we evaluate them in a critical point, we obtain

$$
\varepsilon \sum_{k=1}^{m}\left\langle\sum_{\ell=0}^{N} c_{k, \ell}^{\varepsilon} P_{\varepsilon} \psi_{\delta_{k}, \xi_{k}}^{\ell}, \frac{\partial \phi_{k}^{\mathbf{d}, \boldsymbol{\tau}, \varepsilon}}{\partial \xi_{i, h}}\right\rangle+\varepsilon\left\langle\sum_{\ell=0}^{N} c_{i, \ell}^{\varepsilon} P_{\varepsilon} \psi_{\delta_{k}, \xi_{k}}^{\ell}, \mu_{i}^{-\frac{1}{p-1}} P_{\varepsilon} \psi_{\delta_{i}, \xi_{i}}^{h}\right\rangle=0
$$

Letting $i$ and $h$ vary, (4.2) and (4.3) provides us a linear homogeneous system of $m(N+1)$ equations in the $m(N+1)$ unknowns $c_{i, h}^{\varepsilon}$. We aim at showing that the system has only the trivial solution; this means that also the projection on $\nabla J_{\varepsilon}\left(V_{1}^{\mathbf{d}, \boldsymbol{\tau}, \varepsilon}, \ldots, V_{m}^{\mathbf{d}, \boldsymbol{\tau}, \varepsilon}\right)$ on $\mathbf{K}_{\mathbf{d}, \boldsymbol{\tau}, \varepsilon}$ vanishes, i.e. $\nabla J_{\varepsilon}\left(V_{1}^{\mathbf{d}, \boldsymbol{\tau}, \varepsilon}, \ldots, V_{m}^{\mathbf{d}, \boldsymbol{\tau}, \varepsilon}\right)=0$ in $H_{0}^{1}\left(\Omega_{\varepsilon}\right)$, and completes the proof. Thus, we consider now (4.2) and (4.3), and we show that the matrix of the coefficients is invertible for $\varepsilon$ small enough.

The last term in both (4.2) and (4.3) can be estimated as in step 1 in Lemma 3.2: recalling that $\varepsilon \simeq \delta_{i}^{2}$ as $\varepsilon \rightarrow 0$, we have for $h=0, \ldots, N$

$$
\varepsilon\left\langle\sum_{\ell=0}^{N} c_{i, \ell}^{\varepsilon} P_{\varepsilon} \psi_{\delta_{k}, \xi_{k}}^{\ell}, \mu_{i}^{-\frac{1}{p-1}} P_{\varepsilon} \psi_{\delta_{i}, \xi_{i}}^{h}\right\rangle=\mu_{i}^{-\frac{1}{p-1}} \sigma_{h h} c_{i, h}^{\varepsilon}+o(1) \sum_{\ell \neq h} c_{i, \ell}^{\varepsilon},
$$

as $\varepsilon \rightarrow 0$, with $\sigma_{h h}>0$ defined by (3.11). Let us now consider the terms involving the derivative of $\phi_{k}^{\mathbf{d}, \boldsymbol{\tau}, \varepsilon}$. By Lemma 3.5, the Cauchy-Schwarz inequality, and recalling again that $\delta_{i}^{2}\left\|P_{\varepsilon} \psi_{\delta_{i}, \xi_{i}}^{\ell}\right\|_{H_{0}^{1}\left(\Omega_{\varepsilon}\right)}^{2} \rightarrow$ $\sigma_{\ell \ell}$, we deduce that

$$
\varepsilon\left|\left\langle P_{\varepsilon} \psi_{\delta_{i}, \xi_{i}}^{\ell}, \frac{\partial \phi_{i}^{\mathbf{d}, \boldsymbol{\tau}, \varepsilon}}{\partial \xi_{i, h}}\right\rangle\right| \leq C \delta_{i}^{2}\left\|P_{\varepsilon} \psi_{\delta_{i}, \xi_{i}}^{\ell}\right\|_{H_{0}^{1}\left(\Omega_{\varepsilon}\right)}\left\|\frac{\partial \phi_{i}^{\mathbf{d}, \boldsymbol{\tau}, \varepsilon}}{\partial \xi_{i, h}}\right\|_{H_{0}^{1}\left(\Omega_{\varepsilon}\right)} \leq C \delta_{i} \varepsilon^{\frac{N-3}{2}} \leq C \varepsilon^{\frac{N-2}{2}},
$$

where we used also the fact that $\delta_{i} \simeq \varepsilon^{\frac{1}{2}}$. The same estimate holds if we consider the derivatives with respect to $\delta_{i}, i=1, \ldots, m$. Thus, plugging (4.4) and (4.5) inside (4.2) and (4.3), we infer that the matrix associated to the system is strictly diagonally dominant, hence invertible, and being homogeneous has only the trivial solution. As observed, this completes the proof.

Due to Lemma 4.1, in order to complete the proof of Theorem 1.1 we have to find a critical point of the reduced functional $\tilde{J}_{\varepsilon}$ in $X_{\eta}$. In this perspective, we need the asymptotic expansion of $J_{\varepsilon}\left(V_{1}^{\mathbf{d}, \boldsymbol{\tau}, \varepsilon}, \ldots, V_{m}^{\mathbf{d}, \boldsymbol{\tau}, \varepsilon}\right)$ as $\varepsilon \rightarrow 0^{+}$. We introduce the real numbers

$$
b_{1}:=\frac{\alpha_{N}^{p+1}}{N} \int_{\mathbb{R}^{N}} \frac{d y}{\left(1+\mid y^{2}\right)^{N}}, \quad b_{2}:=\frac{\alpha_{N}^{p+1}}{2} \int_{\mathbb{R}^{N}} \frac{d y}{\left(1+|y|^{2}\right)^{\frac{N+2}{2}}},
$$


and the functions $\Gamma: \mathbb{R}^{N} \rightarrow \mathbb{R}, \Psi: X_{\eta} \rightarrow \mathbb{R}$ defined by

$$
\begin{aligned}
\Gamma(x) & :=\int_{\mathbb{R}^{N}} \frac{d y}{|y+x|^{N-2}\left(1+|y|^{2}\right)^{\frac{N+2}{2}}} \\
\Psi(\mathbf{d}, \boldsymbol{\tau}) & :=\sum_{i=1}^{m} \mu_{i}^{-\frac{2}{p-1}}\left[b_{2} H\left(a_{i}, a_{i}\right) d_{i}^{N-2}+\frac{\alpha_{N}^{p+1} r_{i}^{N-2}}{2} \frac{\Gamma\left(\tau_{i}\right)}{d_{i}^{N-2}\left(1+\left|\tau_{i}\right|^{2}\right)^{\frac{N-2}{2}}}\right] .
\end{aligned}
$$

Proposition 4.2. We have

$$
\tilde{J}_{\varepsilon}(\mathbf{d}, \boldsymbol{\tau})=\left(\sum_{i=1}^{m} \mu_{i}^{-\frac{2}{p-1}}\right) b_{1}+\Psi(\mathbf{d}, \boldsymbol{\tau}) \varepsilon^{\frac{N-2}{2}}+R_{\varepsilon}(\mathbf{d}, \boldsymbol{\tau}),
$$

with $R_{\varepsilon}=o\left(\varepsilon^{\frac{N-2}{2}}\right) \mathcal{C}^{1}$-uniformly in $X_{\eta}$ as $\varepsilon \rightarrow 0$.

Here and what follows, we write that $f_{\varepsilon}=o\left(\varepsilon^{\alpha}\right) \mathcal{C}^{k}$-uniformly in $X_{\eta}$ as $\varepsilon \rightarrow 0$ if

$$
\lim _{\varepsilon \rightarrow 0} \frac{\left\|f_{\varepsilon}\right\|_{\mathcal{C}^{k}\left(X_{\eta}\right)}}{\varepsilon^{\alpha}}=0
$$

The proof of the proposition takes most of the rest of the section. In order to keep the notation short, in what follows we sometimes write $P, U_{i}, V_{i}, \phi_{i}$ and $\|\cdot\|$ instead of $P_{\varepsilon}, U_{\delta_{i}, \xi_{i}}, V_{i}^{\mathbf{d}, \boldsymbol{\tau}, \varepsilon}, \phi_{i}^{\mathbf{d}, \boldsymbol{\phi}, \varepsilon}$ and $\|\cdot\|_{H_{0}^{1}\left(\Omega_{\varepsilon}\right)}$, respectively.

Let $I_{\varepsilon, \mu}: H_{0}^{1}\left(\Omega_{\varepsilon}\right) \rightarrow \mathbb{R}$ be defined by

$$
I_{\varepsilon}(u)=\frac{1}{2} \int_{\Omega_{\varepsilon}}|\nabla u|^{2}-\int_{\Omega_{\varepsilon}} \mu F(u), \quad I_{\varepsilon}:=I_{\varepsilon, 1} .
$$

Then, by the Lagrange theorem,

$$
\begin{aligned}
\tilde{J}_{\varepsilon}(\mathbf{d}, \boldsymbol{\tau})= & \sum_{i} I_{\varepsilon, \mu_{i}}\left(V_{i}\right)-\frac{2}{p+1} \sum_{i<j} \int_{\Omega_{\varepsilon}} \beta_{i j}\left|V_{i}\right|^{\frac{p+1}{2}}\left|V_{j}\right|^{\frac{p+1}{2}} \\
= & \sum_{i} \mu_{i}^{-\frac{2}{p+1}} I_{\varepsilon}\left(P U_{i}\right)+\frac{1}{2} \sum_{i} \int_{\Omega_{\varepsilon}} 2 \mu_{i}^{-\frac{1}{p-1}} \nabla\left(P U_{i}\right) \cdot \nabla \phi_{i}+\left|\nabla \phi_{i}\right|^{2} \\
& -\sum_{i} \int_{\Omega_{\varepsilon}} \mu_{i}\left(F\left(\mu_{i}^{-\frac{1}{p-1}} P U_{i}+\phi_{i}\right)-F\left(\mu_{i}^{-\frac{1}{p-1}} P U_{i}\right)\right) \\
& -\frac{2}{p+1} \sum_{i<j} \beta_{i j} \int_{\Omega_{\varepsilon}}\left|\mu_{i}^{-\frac{1}{p-1}} P U_{i}\right|^{\frac{p+1}{2}}\left|\mu_{j}^{-\frac{1}{p-1}} P U_{j}\right|^{\frac{p+1}{2}} \\
& -\sum_{i<j} \beta_{i j} \int_{\Omega_{\varepsilon}}\left(\left|\mu_{i}^{-\frac{1}{p-1}} P U_{i}+\eta_{i} \phi_{i}\right|^{\frac{p+1}{2}}\left|\mu_{j}^{-\frac{1}{p-1}} P U_{j}+\eta_{j} \phi_{j}\right|^{\frac{p-1}{2}} \phi_{j}\right. \\
& \left.\quad+\left|\mu_{i}^{-\frac{1}{p-1}} P U_{i}+\eta_{i} \phi_{i}\right|^{\frac{p-1}{2}}\left|\mu_{j}^{-\frac{1}{p-1}} P U_{j}+\eta_{j} \phi_{j}\right|^{\frac{p+1}{2}} \phi_{i}\right)
\end{aligned}
$$

where $\eta_{i}, \eta_{j}$ denote continuous functions with values in $[0,1]$.

We start considering the asymptotic expansion of $I_{\varepsilon}\left(P_{\varepsilon} U_{\delta_{i}, \xi_{i}}\right)$.

Lemma 4.3. For every $i=1, \ldots, m$, it results that

$$
I_{\varepsilon}\left(P_{\varepsilon} U_{\delta_{i}, \xi_{i}}\right)=b_{1}+\left[b_{2} H\left(a_{i}, a_{i}\right) d_{i}^{N-2}+\frac{\alpha_{N}^{p+1} r_{i}^{N-2}}{2} \frac{\Gamma\left(\tau_{i}\right)}{d_{i}^{N-2}\left(1+\left|\tau_{i}\right|^{2}\right)^{\frac{N-2}{2}}}\right] \varepsilon^{\frac{N-2}{2}}+o\left(\varepsilon^{\frac{N-2}{2}}\right)
$$


as $\varepsilon \rightarrow 0, \mathcal{C}^{1}$-uniformly in $(\mathbf{d}, \boldsymbol{\tau}) \in X_{\eta}$, where the real numbers $b_{1}, b_{2}>0$ and the function $\Gamma: \mathbb{R}^{N} \rightarrow \mathbb{R}$ are defined by (4.6) and (4.7).

Proof. At first, using the definition of $P_{\varepsilon} U_{\delta_{i}, \xi_{i}}$, we observe that

$$
\begin{aligned}
I_{\varepsilon}\left(P_{\varepsilon} U_{\delta_{i}, \xi_{i}}\right)= & \frac{1}{2} \int_{\Omega_{\varepsilon}} P_{\varepsilon} U_{\delta_{i}, \xi_{i}} U_{\delta_{i}, \xi_{i}}^{p}-\frac{1}{p+1} \int_{\Omega_{\varepsilon}}\left(P_{\varepsilon} U_{\delta_{i}, \xi_{i}}\right)^{p+1} \\
= & \left(\frac{1}{2}-\frac{1}{p+1}\right) \int_{\Omega_{\varepsilon}} U_{\delta_{i}, \xi_{i}}^{p+1}+\frac{1}{2} \int_{\Omega_{\varepsilon}} U_{\delta_{i}, \xi_{i}}^{p}\left(P_{\varepsilon} U_{\delta_{i}, \xi_{i}}-U_{\delta_{i}, \xi_{i}}\right) \\
& \quad-\frac{1}{p+1} \int_{\Omega_{\varepsilon}}\left(\left(P_{\varepsilon} U_{\delta_{i}, \xi_{i}}\right)^{p+1}-U_{\delta_{i}, \xi_{i}}^{p+1}\right) \\
= & \frac{1}{N} \int_{\Omega_{\varepsilon}} U_{\delta_{i}, \xi_{i}}^{p+1}-\frac{1}{2} \int_{\Omega_{\varepsilon}} U_{\delta_{i}, \xi_{i}}^{p}\left(P_{\varepsilon} U_{\delta_{i}, \xi_{i}}-U_{\delta_{i}, \xi_{i}}\right) \\
& \quad-\frac{p}{2} \int_{\Omega_{\varepsilon}}\left(U_{\delta_{i}, \xi_{i}}+\eta_{i}\left(P_{\varepsilon} U_{\delta_{i}, \xi_{i}}-U_{\delta_{i}, \xi_{i}}\right)\right)^{p-1}\left(P_{\varepsilon} U_{\delta_{i}, \xi_{i}}-U_{\delta_{i}, \xi_{i}}\right)^{2}
\end{aligned}
$$

for a function $\eta_{i}$ with values in $[0,1]$. Now we estimate separately the three terms on the right hand side. Recalling that $\Omega_{\varepsilon}=\Omega \backslash \bigcup_{i} B_{r_{i} \varepsilon}\left(a_{i}\right)$, the first term gives

$$
\int_{\Omega_{\varepsilon}} U_{\delta_{i}, \xi_{i}}^{p+1}=\alpha_{N}^{p+1} \int_{\frac{\Omega_{\varepsilon}-\xi_{i}}{\delta_{i}}} \frac{d y}{\left(1+|y|^{2}\right)^{N}}=b_{1}+O\left(\left(\frac{\varepsilon}{\delta_{i}}\right)^{N}+\delta_{i}^{N}\right) .
$$

To treat the second term, we use Lemma A.1 $\left(R=R_{\varepsilon, d_{i}, \tau_{i}}\right)$ :

$$
\begin{aligned}
\int_{\Omega_{\varepsilon}} U_{\delta_{i}, \xi_{i}}^{p} & \left(P_{\varepsilon} U_{\delta_{i}, \xi_{i}}-U_{\delta_{i}, \xi_{i}}\right)=\alpha_{N}^{p} \int_{\Omega_{\varepsilon}} R_{\varepsilon, \delta_{i}, \xi_{i}} U_{\delta_{i}, \xi_{i}}^{p} \\
& -\alpha_{N}^{p+1} \int_{\Omega_{\varepsilon}}\left(\delta_{i}^{\frac{N-2}{2}} H\left(x, \xi_{i}\right)+\frac{\delta_{i}^{-\frac{N-2}{2}}}{\left(1+|\tau|^{2}\right)^{\frac{N-2}{2}}}\left(\frac{r_{i} \varepsilon}{|x-a|}\right)^{N-2}\right) U_{\delta_{i}, \xi_{i}}^{p}
\end{aligned}
$$

Now, by dominated convergence

$$
\begin{aligned}
\int_{\Omega_{\varepsilon}} \delta_{i}^{\frac{N-2}{2}} H\left(x, \xi_{i}\right) U_{\delta_{i}, \xi_{i}}^{p} & =\int_{\frac{\Omega_{\varepsilon}-\xi_{i}}{\delta_{i}}} \delta_{i}^{N-2} \frac{H\left(\xi_{i}+\delta_{i} y, \xi_{i}\right)}{\left(1+|y|^{2}\right)^{\frac{N+2}{2}}} d y \\
& =b_{2} H\left(a_{i}, a_{i}\right) \delta_{i}^{N-2}+o\left(\delta_{i}^{N-2}\right),
\end{aligned}
$$

and

$$
\begin{aligned}
\int_{\Omega_{\varepsilon}} \frac{\delta_{i}^{-\frac{N-2}{2}}}{\left(1+\left|\tau_{i}\right|^{2}\right)^{\frac{N-2}{2}}} & \left(\frac{r_{i} \varepsilon}{|x-a|}\right)^{N-2} U_{\delta_{i}, \xi_{i}}^{p} \\
& =\frac{1}{\left(1+\left|\tau_{i}\right|^{2}\right)^{\frac{N-2}{2}}}\left(\frac{r_{i} \varepsilon}{\delta_{i}}\right)^{N-2} \int_{\frac{\Omega_{\varepsilon}-\xi_{i}}{\delta_{i}}} \frac{d y}{\left|y+\tau_{i}\right|^{N-2}\left(1+|y|^{2}\right)^{\frac{N+2}{2}}} \\
& =\left(\frac{\varepsilon}{\delta_{i}}\right)^{N-2} \frac{r_{i}^{N-2} \Gamma\left(\tau_{i}\right)}{\left(1+\left|\tau_{i}\right|^{2}\right)^{\frac{N-2}{2}}}+o\left(\left(\frac{\varepsilon}{\delta_{i}}\right)^{N-2}\right),
\end{aligned}
$$

as $\varepsilon \rightarrow 0$. Also, by Lemma A.1 the term with $R_{\varepsilon, \delta_{i}, \xi_{i}}$ is of lower order with respect to those in (4.12) and (4.13), and can be absorbed in the small $o$ therein. 
Since $\delta_{i} \simeq \varepsilon^{\frac{1}{2}}$, it remains to estimate only the last term in (4.9), and this can be done using [14, Lemma 3.2]: as $0 \leq P_{\varepsilon} U_{\delta_{i}, \xi_{i}} \leq U_{\delta_{i}, \xi_{i}}$, we have

$$
\begin{array}{r}
\left|\int_{\Omega_{\varepsilon}}\left(U_{\delta_{i}, \xi_{i}}+\eta_{i}\left(P_{\varepsilon} U_{\delta_{i}, \xi_{i}}-U_{\delta_{i}, \xi_{i}}\right)\right)^{p-1}\left(P_{\varepsilon} U_{\delta_{i}, \xi_{i}}-U_{\delta_{i}, \xi_{i}}\right)^{2}\right| \\
\leq C \int_{\Omega_{\varepsilon}} U_{\delta_{i}, \xi_{i}}^{p-1}\left(P_{\varepsilon} U_{\delta_{i}, \xi_{i}}-U_{\delta_{i}, \xi_{i}}\right)^{2}=o\left(\varepsilon^{\frac{N-2}{2}}\right)
\end{array}
$$

Collecting all the previous computations, we infer that the expansion in the thesis holds $\mathcal{C}^{0}$ uniformly as $\varepsilon \rightarrow 0$. The estimates for the derivatives can be obtained in a similar way.

Coming back to (4.8), we now show that the other terms are perturbation of $I_{\varepsilon}\left(P_{\varepsilon} U_{\delta_{i}, \xi_{i}}\right)$.

Lemma 4.4. Let $R_{1, \varepsilon}: X_{\eta} \rightarrow \mathbb{R}$ be defined by

$$
R_{1, \varepsilon}(\mathbf{d}, \boldsymbol{\tau}):=\tilde{J}_{\varepsilon}(\mathbf{d}, \boldsymbol{\tau})-\sum_{i=1}^{m} \mu_{i}^{-\frac{2}{p-1}} I_{\varepsilon}\left(P_{\varepsilon} U_{\delta_{i}, \xi_{i}}\right)
$$

Then $R_{1, \varepsilon}=o\left(\varepsilon^{\frac{N-2}{2}}\right) \mathcal{C}^{1}$-uniformly in $X_{\eta}$ as $\varepsilon \rightarrow 0$.

Proof. Using the definitions of $P U_{i}$ and of $F$, we have

$$
\begin{aligned}
{\left[\frac{1}{2} \int_{\Omega_{\varepsilon}} 2 \mu_{i}^{-\frac{1}{p-1}}\right.} & \left.\nabla P U_{i} \cdot \nabla \phi_{i}-\int_{\Omega_{\varepsilon}} \mu_{i}\left(F\left(\mu_{i}^{-\frac{1}{p-1}} P U_{i}+\phi_{i}\right)-F\left(\mu_{i}^{-\frac{1}{p-1}} P U_{i}\right)\right)\right] \\
& =\int_{\Omega_{\varepsilon}} \mu_{i}^{-\frac{1}{p-1}}\left(U_{i}^{p}-P U_{i}^{p}\right) \phi_{i} \\
& -\int_{\Omega_{\varepsilon}} \mu_{i}\left(F\left(\mu_{i}^{-\frac{1}{p-1}} P U_{i}+\phi_{i}\right)-F\left(\mu_{i}^{-\frac{1}{p-1}} P U_{i}\right)-F^{\prime}\left(\mu_{i}^{-\frac{1}{p-1}} P U_{i}\right) \phi_{i}\right) .
\end{aligned}
$$

The first term on the right hand side can be controlled using Corollary A.2, Lemmas A.4, A.6 and A.7, the fact that $0 \leq P U_{i} \leq U_{i}$, and the Hölder and the Sobolev inequalities:

$$
\begin{aligned}
& \left|\int_{\Omega_{\varepsilon}}\left(U_{i}^{p}-P U_{i}^{p}\right) \phi_{i}\right| \leq C \int_{\Omega_{\varepsilon}} U_{i}^{p-1}\left|P U_{i}-U_{i} \| \phi_{i}\right| \\
& \quad \leq C\left(\int_{\Omega_{\varepsilon}} U_{i}^{\frac{8 N}{(N-2)(N+2)}}\left(\delta_{i}^{\frac{N-2}{2}}+\frac{\delta_{i}^{\frac{3}{2} N-3}}{\left|x-a_{i}\right|^{N-2}}\right)\right)^{\frac{N+2}{2 N}}\left\|\phi_{i}\right\| \\
& \quad \leq C \delta_{i}^{\frac{N-2}{2}}\left(\int_{\Omega_{\varepsilon}} U_{i}^{\frac{8 N}{(N-2)(N+2)}}\right)^{\frac{N+2}{2 N}}\left\|\phi_{i}\right\|+C \delta_{i}^{\frac{3}{2} N-3}\left(\int_{\Omega_{\varepsilon}} \frac{U_{i}^{\frac{8 N}{(N-2)(N+2)}}}{\left|x-a_{i}\right|^{\frac{2 N(N-2)}{N+2}}}\right)^{\frac{N+2}{2 N}}\left\|\phi_{i}\right\| \\
& \quad \leq C \delta_{i}^{\frac{N-2}{2}}\left(\delta_{i}^{\frac{N(N-2)}{N+2}}\right)^{\frac{N+2}{2 N}}\left\|\phi_{i}\right\|+C \delta_{i}^{\frac{3}{2} N-3}\left(\delta_{i}^{\frac{N(2-N)}{N+2}}\right)^{\frac{N+2}{2 N}}\left\|\phi_{i}\right\| \leq C \delta_{i}^{N-2}\left\|\phi_{i}\right\|=o\left(\varepsilon^{\frac{N-2}{2}}\right)
\end{aligned}
$$

uniformly in $X_{\eta}$ as $\varepsilon \rightarrow 0$, where the last equality follows by Proposition 3.1. Regarding the second term on the right hand side in (4.14), by the Lagrange theorem there exists a function $\eta_{i}$ with 
values in $[0,1]$ such that

$$
\begin{aligned}
\int_{\Omega_{\varepsilon}} & \left|F\left(\mu_{i}^{-\frac{1}{p-1}} P U_{i}+\phi_{i}\right)-F\left(\mu_{i}^{-\frac{1}{p-1}} P U_{i}\right)-F^{\prime}\left(\mu_{i}^{-\frac{1}{p-1}} P U_{i}\right) \phi_{i}\right| \\
& =\int_{\Omega_{\varepsilon}}\left|F^{\prime \prime}\left(\mu_{i}^{-\frac{1}{p-1}} P U_{i}+\eta_{i} \phi_{i}\right)\right| \phi_{i}^{2} \leq C \int_{\Omega_{\varepsilon}}\left(\left(P U_{i}\right)^{p-1} \phi_{i}^{2}+\left|\phi_{i}\right|^{p+1}\right) \\
& \leq C \int_{\Omega_{\varepsilon}} U_{i}^{p-1} \phi_{i}^{2}+C\left|\phi_{i}\right|_{2^{*}}^{2^{*}} \leq C\left|U_{i}\right|_{2^{*}}^{p-1}\left|\phi_{i}\right|_{2^{*}}^{2}+C\left\|\phi_{i}\right\|^{2 *} \\
& \leq C\left\|\phi_{i}\right\|^{2}+\left\|\phi_{i}\right\|^{2^{*}}=o\left(\varepsilon^{\frac{N-2}{2}}\right)
\end{aligned}
$$

uniformly in $X_{\eta}$ as $\varepsilon \rightarrow 0$. To sum up, the left hand side in (4.14) is $o\left(\varepsilon^{\frac{N-2}{2}}\right)$ uniformly in $X_{\eta}$ as $\varepsilon \rightarrow 0$.

To estimate the other terms on the right hand side in (4.8), we use Lemmas A.6 and A.8, the fact that $0 \leq P_{\varepsilon} U_{\delta, \xi} \leq U_{\delta, \xi}$, the ansatz (2.5), and the estimate in Proposition 3.1:

$$
\begin{gathered}
\left|\int_{\Omega_{\varepsilon}} P U_{i}^{\frac{p+1}{2}} P U_{j}^{\frac{p+1}{2}}\right| \leq \int_{\Omega_{\varepsilon}} U_{i}^{\frac{N}{N-2}} U_{j}^{\frac{N}{N-2}} \leq C \delta_{i}^{\frac{N}{2}} \delta_{j}^{\frac{N}{2}}\left(\left|\log \delta_{i}\right|+\left|\log \delta_{j}\right|\right)=o\left(\varepsilon^{\frac{N-2}{2}}\right), \\
\int_{\Omega_{\varepsilon}}\left|\mu_{i}^{-\frac{1}{p-1}} P U_{i}+\eta_{i} \phi_{i}\right|^{\frac{p+1}{2}}\left|\mu_{j}^{-\frac{1}{p-1}} P U_{j}+\eta_{j} \phi_{j}\right|^{\frac{p-1}{2}}\left|\phi_{j}\right| \\
\leq C \int_{\Omega_{\varepsilon}}\left(U_{i}^{\frac{p+1}{2}} U_{j}^{\frac{p-1}{2}}\left|\phi_{j}\right|+\left|\phi_{i}\right|^{\frac{p+1}{2}} U_{j}^{\frac{p-1}{2}}\left|\phi_{j}\right|+U_{i}^{\frac{p-1}{2}}\left|\phi_{j}\right|^{\frac{p+1}{2}}+\left|\phi_{i}\right|^{\frac{p+1}{2}}\left|\phi_{j}\right|^{\frac{p+1}{2}}\right) \\
\leq C\left(\int_{\Omega_{\varepsilon}} U_{i}^{\frac{2 N^{2}}{(N+2)(N-2)}} U_{j}^{\frac{4 N}{(N+2)(N-2)}}\right)^{\frac{N+2}{2 N}}\left|\phi_{j}\right|_{2^{*}}+C\left|\phi_{i}\right|_{2^{*}}^{\frac{p+1}{2}}\left|U_{j}\right|_{2^{*}}^{\frac{p-1}{2}}\left|\phi_{j}\right|_{2^{*}} \\
\quad+C\left|U_{i}\right|_{2^{*}}^{\frac{p-1}{2}}\left|\phi_{j}\right|_{2^{*}}^{\frac{p+1}{2}}+C+\left|\phi_{i}\right|_{2^{*}}^{\frac{p+1}{2}}\left|\phi_{j}\right|_{2^{*}}^{\frac{p+1}{2}} \\
\leq C \delta_{i} \delta_{j}\left\|\phi_{j}\right\|+C\left\|\phi_{i}\right\|^{\frac{p+1}{2}}\left\|\phi_{j}\right\|+C\left\|\phi_{j}\right\|^{\frac{p+1}{2}}+C\left\|\phi_{i}\right\|^{\frac{p+1}{2}}\left\|\phi_{j}\right\|^{\frac{p+1}{2}}=o\left(\varepsilon^{\frac{N-2}{2}}\right),
\end{gathered}
$$

and analogously

$$
\int_{\Omega_{\varepsilon}}\left|\mu_{i}^{-\frac{1}{p-1}} P U_{i}+\eta_{i} \phi_{i}\right|^{\frac{p-1}{2}}\left|\mu_{j}^{-\frac{1}{p-1}} P U_{j}+\eta_{j} \phi_{j}\right|^{\frac{p+1}{2}}\left|\phi_{i}\right|=o\left(\varepsilon^{\frac{N-2}{2}}\right)
$$

uniformly in $X_{\eta}$ as $\varepsilon \rightarrow 0$.

Collecting together the previous estimates, we deduce that $R_{1, \varepsilon}=o\left(\varepsilon^{\frac{N-2}{2}}\right) \mathcal{C}^{0}$-uniformly as $\varepsilon \rightarrow 0$. The estimates on the derivatives can be obtained in a similar way.

Proposition 4.2 follows from Lemmas 4.3 and 4.4 straightforwardly, and allows us to complete the proof of Theorem 1.1: we show that for every $\varepsilon>0$ small enough the function $\tilde{J}_{\varepsilon}$ has a critical point in $X_{\eta}$. A crucial lemma is the following.

Lemma 4.5. The function $\Psi$ defined in (4.7) has a non-degenrate critical point $(\tilde{\mathbf{d}}, 0) \in X_{\eta}$, provided $\eta>0$ was chosen small enough at the beginning. 
Proof. We have

$$
\begin{aligned}
\partial_{d_{i}} \Psi(\mathbf{d}, \boldsymbol{\tau}) & =(N-2) \mu_{i}^{-\frac{2}{p-1}} b_{2} H\left(a_{i}, a_{i}\right) d_{i}^{N-3}-(N-2) \frac{\alpha_{N}^{p+1} \mu_{i}^{-\frac{2}{p-1}} r_{i}^{N-2}}{2} \frac{\Gamma\left(\tau_{i}\right)}{d_{i}^{N-1}\left(1+\left|\tau_{i}\right|^{2}\right)^{\frac{N-2}{2}}} \\
\partial_{\tau_{i, h}} \Psi(\mathbf{d}, \boldsymbol{\tau}) & =\frac{\alpha_{N}^{p+1} \mu_{i}^{-\frac{2}{p-1}} r_{i}^{N-2}}{2 d_{i}^{N-2}}\left(\frac{\partial_{\tau_{i, h}} \Gamma\left(\tau_{i}\right)}{\left(1+\left|\tau_{i}\right|^{2}\right)^{\frac{N-2}{2}}}-\frac{(N-2) \Gamma\left(\tau_{i}\right) \tau_{i, h}}{\left(1+\left|\tau_{i}\right|^{2}\right)^{\frac{N}{2}}}\right) .
\end{aligned}
$$

As proved in [14, Lemma 4.1], the function $\Gamma$ has a non-degenerate maximum in 0 (the Hessian matrix is diagonal with all negative entries), and hence we deduce that for every $\mathbf{d}$

$$
\nabla_{\boldsymbol{\tau}} \Psi(\mathbf{d}, 0)=0 .
$$

Thus, to find a critical point of $\Psi$, it is sufficient to find $\mathbf{d}$ with $\eta<d_{i}<\eta^{-1}$ for every $i$ such that

$$
\nabla_{\mathbf{d}} \Psi(\mathbf{d}, 0)=0 .
$$

The existence of such critical point follows straightforwardly by the fact that

$$
\Psi(\mathbf{d}, 0)=\sum_{i} g_{i}\left(d_{i}\right), \quad \text { with } \quad g_{i}\left(d_{i}\right):=\tilde{b}_{i, 1} d_{i}^{N-2}+\frac{\tilde{b}_{i, 2}}{d_{i}^{N-2}}
$$

for suitable positive constants $\tilde{b}_{i, 1}, \tilde{b}_{i, 2}$, so that $\mathbf{d} \mapsto \Psi(\mathbf{d}, 0)$ admits a global minimum $\tilde{\mathbf{d}}$ in the set $\left\{\eta<d_{i}<\eta^{-1}\right\}$ (at least for $\eta>0$ small enough).

Now we show that $(\tilde{\mathbf{d}}, 0)$ is non-degenerate. The Hessian matrix $D^{2} \Psi(\tilde{\mathbf{d}}, 0)$ can be divided in blocks in the following way:

$$
D^{2} \Psi(\tilde{\mathbf{d}}, 0)=\left(\begin{array}{lr}
D_{\mathbf{d}}^{2} \Psi(\tilde{\mathbf{d}}, 0) & \left(\partial_{\tau_{i, h}, d_{j}} \Psi(\mathbf{d}, 0)\right) \\
\left(\partial_{\tau_{i, h}, d_{j}} \Psi(\mathbf{d}, 0)\right) & D_{\boldsymbol{\tau}}^{2} \Psi(\tilde{\mathbf{d}}, 0)
\end{array}\right)
$$

Recalling that 0 is a non-degenerate maximum for the function $\Gamma$, it is not difficult to check by direct computations that the matrix $D_{\tau}^{2} \Psi(\tilde{\mathbf{d}}, 0)$ is diagonal and negative definite. Moreover, for every $i, j=1, \ldots, m$ and $h=1, \ldots, N$ we have $\partial_{\tau_{i, h}, d_{j}} \Psi(\tilde{\mathbf{d}}, 0)=0$, and hence to prove the nondegeneracy of $(\tilde{\mathbf{d}}, 0)$ it remains only to analyze the block $D_{\mathbf{d}}^{2} \Psi(\tilde{\mathbf{d}}, 0)$. It is clear that this is another diagonal matrix, with

$$
\partial_{d_{i}, d_{i}} \Psi(\tilde{\mathbf{d}}, 0)=\frac{2}{\mu_{i}} b_{2} H\left(a_{i}, a_{i}\right)+6 \frac{\alpha_{4}^{4} r_{i}^{2}}{2 \mu_{i}} \frac{\Gamma(0)}{\tilde{d}_{i}^{4}} \quad \text { if } N=4
$$

and

$$
\partial_{d_{i}, d_{i}} \Psi(\tilde{\mathbf{d}}, 0)=2 \frac{\alpha_{3}^{6} r_{i}}{2 \mu_{i}^{\frac{1}{2}}} \frac{\Gamma(0)}{\tilde{d}_{i}^{3}} \quad \text { if } N=3 .
$$

In any case, $D_{\mathbf{d}}^{2} \Psi(\tilde{\mathbf{d}}, 0)$ is diagonal and positive definite, and hence we deduce that $(\tilde{\mathbf{d}}, 0)$ is a non-degenerate saddle-point for $\Psi$.

Conclusion of the proof of Theorem 1.1. Thanks to Lemma 4.1, we prove that for $\varepsilon>0$ small the function $\tilde{J}_{\varepsilon}$ has a critical point in $X_{\eta}$. By Proposition 4.2, this amounts to find a solution of the algebraic system

$$
\left\{\begin{array}{l}
\nabla_{\mathbf{d}} \Psi(\mathbf{d}, \boldsymbol{\tau}) \varepsilon^{\frac{N-2}{2}}+\nabla_{\mathbf{d}} R_{\varepsilon}(\mathbf{d}, \boldsymbol{\tau})=0 \\
\nabla_{\boldsymbol{\tau}} \Psi(\mathbf{d}, \boldsymbol{\tau}) \varepsilon^{\frac{N-2}{2}}+\nabla_{\boldsymbol{\tau}} R_{\varepsilon}(\mathbf{d}, \boldsymbol{\tau})=0
\end{array}\right.
$$


with $\nabla_{\mathbf{d}} R_{\varepsilon}(\mathbf{d}, \boldsymbol{\tau}), \nabla_{\boldsymbol{\tau}} R_{\varepsilon}(\mathbf{d}, \boldsymbol{\tau})=o\left(\varepsilon^{\frac{N-2}{2}}\right)$ as $\varepsilon \rightarrow 0, \mathcal{C}^{0}$-uniformly in $X_{\eta}$. The previous system can be rewritten as

$$
\left\{\begin{array}{l}
\nabla_{\mathbf{d}} \Psi(\mathbf{d}, \boldsymbol{\tau})+g_{1}(\mathbf{d}, \tau, \varepsilon)=0 \\
\nabla_{\boldsymbol{\tau}} \Psi(\mathbf{d}, \boldsymbol{\tau})+g_{2}(\mathbf{d}, \tau, \varepsilon)=0
\end{array}\right.
$$

with $g_{1}, g_{2}=o(1)$ as $\varepsilon \rightarrow 0, \mathcal{C}^{0}$-uniformly in $(\mathbf{d}, \boldsymbol{\tau}) \in X_{\eta}$. Now, let us define the two maps

$$
\Lambda_{0}(\mathbf{d}, \boldsymbol{\tau}):=\left(\begin{array}{c}
\nabla_{\mathbf{d}} \Psi(\mathbf{d}, \boldsymbol{\tau}) \\
\nabla_{\boldsymbol{\tau}} \Psi(\mathbf{d}, \boldsymbol{\tau})
\end{array}\right), \quad \Lambda_{1, \varepsilon}(\mathbf{d}, \boldsymbol{\tau}):=\left(\begin{array}{c}
\nabla_{\mathbf{d}} \Psi(\mathbf{d}, \boldsymbol{\tau})+g_{1}(\mathbf{d}, \boldsymbol{\tau}, \varepsilon) \\
\nabla_{\boldsymbol{\tau}} \Psi(\mathbf{d}, \boldsymbol{\tau})+g_{2}(\mathbf{d}, \boldsymbol{\tau}, \varepsilon)
\end{array}\right) .
$$

The zeros of $\Lambda_{1, \varepsilon}$ are critical point of the reduced functional $\tilde{J}_{\varepsilon}$, and hence gives solutions to (1.4). By Lemma 4.5, we know that $\Lambda_{0}$ has a zero in $(\tilde{\mathbf{d}}, 0)$, and by non-degeneracy there exists a neighbourhood $U \subset X_{\eta}$ of $(\tilde{\mathbf{d}}, 0)$ such that $(\tilde{\mathbf{d}}, 0)$ is the unique 0 of $\Lambda_{0}$ in $\bar{U}$, and $\operatorname{deg}\left(\Lambda_{0}, U, 0\right) \neq 0$.

Let $H_{\varepsilon}:[0,1] \times U \rightarrow \mathbb{R}^{m} \times \mathbb{R}^{N m}$ be defined by

$$
H_{\varepsilon}(t, \mathbf{d}, \boldsymbol{\tau}):=\Lambda_{0}(\mathbf{d}, \boldsymbol{\tau})+t\left(\begin{array}{c}
g_{1}(\mathbf{d}, \boldsymbol{\tau}, \varepsilon) \\
g_{2}(\mathbf{d}, \boldsymbol{\tau}, \varepsilon)
\end{array}\right) .
$$

This is an homotopy between $\Lambda_{0}$ and $\Lambda_{1, \varepsilon}$, and since $g_{1}, g_{2} \rightarrow 0$ as $\varepsilon \rightarrow 0$ uniformly in $X_{\eta}$, is such that $H_{\varepsilon}(t, \mathbf{d}, \boldsymbol{\tau}) \neq 0$ for every $t \in[0,1],(\mathbf{d}, \boldsymbol{\tau}) \in \partial U$, at least for $\varepsilon>0$ small enough. As a consequence, by the homotopy-invariance property of the topological degree, we conclude that

$$
\operatorname{deg}\left(\Lambda_{0}, U, 0\right)=\operatorname{deg}\left(\Lambda_{1, \varepsilon}, H_{\varepsilon}(1, U), 0\right) \neq 0,
$$

and hence $\Lambda_{1, \varepsilon}$ has a zero in $H(1, U)$. That is, $\tilde{J}_{\varepsilon}$ has a critical point, as desired. This completes the proof of the existence of a solution $\left(u_{1, \varepsilon}, \ldots, u_{m, \varepsilon}\right)$ to system $(2.1)$.

It remains to show that, if necessary replacing $\bar{\beta}$ with a smaller quantity, $u_{i, \varepsilon}>0$ in $\Omega_{\varepsilon}$ for every $i$, so that in particular $\left(u_{1, \varepsilon}, \ldots, u_{m, \varepsilon}\right)$ solves (1.4).

We start from the case $N=4$, in which case we have $\beta_{i j}<\bar{\beta}$ for every $i \neq j$. This case can be treated exactly as in [23, Conclusion of the proof of Theorem 1.1]. ${ }^{1}$

Regarding the case $N=3$, the positivity of the solutions (without any assumption on $\beta_{i j}$ ) can be obtained replacing system (2.1) with

$$
\left\{\begin{array}{lll}
-\Delta u_{i}=\mu_{i} f\left(u_{i}\right)+\sum_{j \neq i} \beta_{i j}\left|u_{j}\right|^{\frac{p+1}{2}}\left|u_{i}\right|^{\frac{p-3}{2}} u_{i}^{+} & \text {in } \Omega_{\varepsilon} \\
u_{i}=0 & \text { on } \partial \Omega_{\varepsilon}, & i=1, \ldots, m .
\end{array}\right.
$$

The key fact is that for $N=3$ the interaction term on the right hand side is of type $F(x, y)=$ $|x| x^{+}|y|^{3}$, which is of class $\mathcal{C}^{1}$, so that the proof we used to deal with system (2.1) in dimensions $N=3,4$ can be used word by word to produce a solution to (4.15) with $u_{i, \varepsilon} \not \equiv 0$ for every $i$. This immediately implies (by the classical maximum principle) that $u_{i, \varepsilon}>0$ in $\Omega_{\varepsilon}$.

Remark 4.6. We stress that the strategy to prove the positivity of $u_{i, \varepsilon}$ in dimension $N=3$ does not work in dimension $N=4$, since for the problem in $\mathbb{R}^{4}$ the interaction term is of type $F(x, y)=x^{+} y^{2}$, which is not $\mathcal{C}^{1}$. We needed the smoothness of $F$ to prove Lemma A.5, which is the key ingredient to estimate the nonlinear part for the equations in $\mathbf{K}_{\mathbf{d}, \boldsymbol{\tau}, \varepsilon}^{\perp}$.

\section{Proof of Theorem 1.2}

We start with some preliminaries about the shape of the approximate solution.

\footnotetext{
${ }^{1}$ Some careful is needed, since to apply the argument in [23] it is required that in the bound $\|\phi\| \leq C \varepsilon^{\frac{N-2}{2}}$, given in Proposition 3.1, the constant $C>0$ is independent on the particular choice of $\beta_{i j}$ with $-\infty<\beta_{i j}<\bar{\beta}$. Going through the proof of Proposition 3.1, it is not difficult to check that this is possible.
} 
5.1. Some preliminaries. Let us consider the 2 components system

$$
\begin{cases}-\Delta v_{1}=\mu_{1} v_{1}^{3}+\beta_{12} v_{1} v_{2}^{2} & \text { in } \mathbb{R}^{4} \\ -\Delta v_{2}=\mu_{2} v_{2}^{3}+\beta_{12} v_{1}^{2} v_{1}^{2} & \text { in } \mathbb{R}^{4} \\ v_{1}, v_{2}>0 & \text { in } \mathbb{R}^{4} \\ v_{1}, v_{2} \in \mathcal{D}^{1,2}\left(\mathbb{R}^{4}\right), & \end{cases}
$$

and let us search for solutions of the form $\left(v_{1}, v_{2}\right)=\left(c_{1} U, c_{2} U\right)$ (where $U=U_{1,0}$ is a standard bubble in $\mathbb{R}^{4}$ centered in 0 and with $\left.\delta=1\right)$, with $c_{1}, c_{2}>0$. This ansatz leads to the algebraic system

$$
\mu_{1} c_{1}^{2}+\beta_{12} c_{2}^{2}=1, \quad \beta_{12} c_{1}^{2}+\mu_{2} c_{2}^{2}=1,
$$

which admits the solution

$$
c_{1}^{2}:=\frac{\beta_{12}-\mu_{2}}{\beta^{2}-\mu_{1} \mu_{2}}, \quad c_{2}^{2}:=\frac{\beta_{12}-\mu_{1}}{\beta^{2}-\mu_{1} \mu_{2}}
$$

if either $-\sqrt{\mu_{1} \mu_{2}}<\beta_{12}<\min \left\{\mu_{1}, \mu_{2}\right\}$, or $\beta_{12}>\max \left\{\mu_{1}, \mu_{2}\right\}$.

Let us consider now the linearization of $(5.1)$ in $\left(c_{1} U, c_{2} U\right)$, namely the linear system

$$
\begin{cases}-\Delta v_{1}=\left(3 \mu_{1} c_{1}^{2}+\beta_{12} c_{2}^{2}\right) U^{2} v_{1}+2 \beta_{12} c_{1} c_{2} U^{2} v_{2}=U^{2}\left(\alpha_{11} v_{1}+\alpha_{12} v_{2}\right) & \text { in } \mathbb{R}^{4} \\ -\Delta v_{2}=2 \beta_{12} c_{1} c_{2} U^{2} v_{1}+\left(3 \mu_{2} c_{2}^{2}+\beta_{12} c_{1}^{2}\right) U^{2} v_{2}=U^{2}\left(\alpha_{21} v_{1}+\alpha_{22} v_{2}\right) & \text { in } \mathbb{R}^{4} .\end{cases}
$$

where

$$
\alpha_{11}:=3 \mu_{1} c_{1}^{2}+\beta_{12} c_{2}^{2}, \quad \alpha_{12}=\alpha_{21}:=2 \beta_{12} c_{1} c_{2}, \quad \alpha_{22}:=3 \mu_{2} c_{2}^{2}+\beta_{12} c_{1}^{2} .
$$

We introduce the $2 \times 2$ matrix $M:=\left(\alpha_{i j}\right)_{i, j=1,2}$, with eigenvalues

$$
\lambda_{2}=\frac{\alpha_{11}+\alpha_{22}+\sqrt{\left(\alpha_{11}-\alpha_{22}\right)^{2}+4 \alpha_{12}^{2}}}{2}, \quad \lambda_{2}=\frac{\alpha_{11}+\alpha_{22}-\sqrt{\left(\alpha_{11}-\alpha_{22}\right)^{2}+4 \alpha_{12}^{2}}}{2} .
$$

Using (5.2), it is not difficult to check by direct computations that $\lambda_{1}=3$.

We consider now the eigenvalue problem:

$$
-\Delta v=\nu U^{2} v, \quad v \in \mathcal{D}^{1,2}\left(\mathbb{R}^{4}\right),
$$

It is well known (see [4, Lemma A.1]) that there exists a sequence of positive eigenvalues $\left\{\nu_{k}\right\}$ with

$$
1=\nu_{1}<3=\nu_{2}<\nu_{3}<\cdots<\nu_{k}<\nu_{k+1}<\ldots,
$$

$\nu_{k} \rightarrow+\infty$.

Lemma 5.1. Let $\left(e_{1}, e_{2}\right)$ be a non-trivial eigenvector of the matrix $M$ associated with $\lambda_{2}$. If $\lambda_{2} \neq \nu_{k}$ for every $k$, then the set of solutions to the linear system (5.4) is 5-dimensional, and is generated by

$$
\left(e_{2},-e_{1}\right) \psi_{1,0}^{h} \quad h=0,1, \ldots, 4
$$

(where $\psi_{1,0}^{h}$ have been defined in (1.14)).

This lemma is a particular case of the forthcoming Lemma 6.1, to which we refer for the proof. Notice that in Lemma 5.2 we express the generators of the set of solutions to (5.4) as $\mathfrak{e}_{1} \psi_{1,0}^{h}$, with $\mathfrak{e}_{1}$ eigenvector associated to the eigenvalue $\lambda_{1}=3$, while here we use an eigenvector associate to $\lambda_{2}$. This is possible since, being eigenvectors associated to different eigenvalues orthogonal, $\left(e_{2},-e_{1}\right)$ is indeed an eigenvector for $\lambda_{1}=3$. 
Proposition 5.2. Under the assumptions of theorem 1.2, the set of solutions to the linear system (5.4) is 5-dimensional, and is generated by

$$
\left(e_{2},-e_{1}\right) \psi_{1,0}^{h} \quad h=0,1, \ldots, 4
$$

(where $\psi_{1,0}^{h}$ have been defined in (1.14)).

Proof. Notice at first that $\lambda_{2}<\lambda_{1}=3$. Then, by Lemma 5.1, to complete the proof is sufficient to show that $\lambda_{2}$ is different from the eigenvalues $\nu_{k}$. Since $\lambda_{2}<3$ and $1=\nu_{1}<\nu_{2}=3$, we have to check that under the assumptions of Theorem 1.2 it results $\lambda_{2} \neq 1$. Using the definition of $\alpha_{i j}$ and the one of $c_{1}, c_{2}$, it is not difficult to check that

$$
\lambda_{1,2}=\frac{6-2 \beta\left(c_{1}^{2}+c_{2}^{2}\right) \pm 2 \beta\left(c_{1}^{2}+c_{2}^{2}\right)}{2} .
$$

If $\lambda_{2}=1$, i.e.

$$
1=3-2 \beta_{12} \frac{2 \beta_{12}-\mu_{1}-\mu_{2}}{\beta^{2}-\mu_{1} \mu_{2}},
$$

then it is not difficult to infer that either $\beta_{12}=\mu_{1}$, or $\beta_{12}=\mu_{2}$. Since in Theorem 1.2 we suppose that either $\beta_{12}<\min \left\{\mu_{1}, \mu_{2}\right\}$, or $\beta_{12}>\max \left\{\mu_{1}, \mu_{2}\right\}$, we have $\lambda_{2} \neq 1$, and the thesis follows.

5.2. The reduction scheme. Once again, we search for solutions of (2.1), which can be rewritten as in (2.2):

$$
u_{i}=i^{*}\left(\mu_{i} f\left(u_{i}\right)+\sum_{j \neq i} \beta_{i j}\left|u_{j}\right|^{\frac{p+1}{2}}\left|u_{i}\right|^{\frac{p-3}{2}} u_{i}\right) .
$$

Let $\eta \in(0,1)$ be small, and let

$$
X_{\eta}:=\left\{(\mathbf{d}, \boldsymbol{\tau})=\left(d_{1}, d_{3}, \tau_{1}, \tau_{3}\right) \in \mathbb{R}^{2} \times\left(\mathbb{R}^{4}\right)^{2}: \eta<d_{1}, d_{3}<\eta^{-1},\left|\tau_{1}\right|,\left|\tau_{3}\right|<\eta^{-1}\right\} .
$$

Our ansatz is that

$$
u_{1}=c_{1} P_{\varepsilon} U_{\delta_{1}, \xi_{1}}+\phi_{1}, \quad u_{2}=c_{2} P_{\varepsilon} U_{\delta_{1}, \xi_{1}}+\phi_{2}, \quad u_{3}=c_{3} P_{\varepsilon} U_{\delta_{3}, \xi_{3}}+\phi_{3}
$$

where for some $(\mathbf{d}, \boldsymbol{\tau})=\left(d_{1}, d_{3}, \tau_{1}, \tau_{3}\right) \in X_{\eta}$ we have

$$
\delta_{i}:=d_{i} \sqrt{\varepsilon}, \quad \xi_{i}:=a_{i}+d_{i} \sqrt{\varepsilon} \tau_{i}, \quad i=1,3 .
$$

Even though we have to deal with only 2 parameters $d_{1}, d_{3}$ and two vectors $\tau_{1}, \tau_{3}$, in order to simplify the notation it is convenient to introduce $d_{2}=d_{1}$ and $\tau_{2}=\tau_{1}$. Analogously, we often write $\delta_{2}=\delta_{1}$ and $\xi_{2}=\xi_{1}$.

Plugging the previous ansatz into (2.2), our problem is transformed in the research of $d_{i}, \tau_{i}$ and $\phi_{i}$ such that (2.6) is satisfied (with $c_{i}$ instead of $\mu_{i}^{-\frac{1}{p-1}}$ ) for $i=1, \ldots, m$, with each equality which takes place in $H_{0}^{1}\left(\Omega_{\varepsilon}\right)$. To proceed, the idea is again to split the space into two orthogonal subspaces, one of them having finite dimension. But in doing this we take into account that $u_{1}$ and $u_{2}$ are concentrating around the same point. Then we define

$$
\begin{aligned}
K_{1}=K_{d_{1}, \tau_{1}, \varepsilon} & :=\operatorname{span}\left\{\left(e_{2},-e_{1}\right) P_{\varepsilon} \psi_{\delta_{1}, \xi_{1}}^{h}: h=0, \ldots, N\right\} \subset H_{0}^{1}\left(\Omega_{\varepsilon}, \mathbb{R}^{2}\right), \\
K_{3}=K_{d_{3}, \tau_{3}, \varepsilon} & :=\operatorname{span}\left\{P_{\varepsilon} \psi_{\delta_{3}, \xi_{3}}^{h}: h=0, \ldots, N\right\} \subset H_{0}^{1}\left(\Omega_{\varepsilon}, \mathbb{R}\right) \\
\mathbf{K}_{\mathbf{d}, \boldsymbol{\tau}, \varepsilon} & :=K_{1} \times K_{3},
\end{aligned}
$$

where $\left(e_{1}, e_{2}\right)$ is an eigenvector with norm 1 of the matrix $M$ associated with $\lambda_{2}$ (defined in (5.6)). Notice that $K_{\mathbf{d}, \boldsymbol{\tau}, \varepsilon}^{\perp}=K_{1}^{\perp} \times K_{3}^{\perp}$. 
If, for $i=1,3$, the symbol $\Pi_{i}=\Pi_{\delta_{i}, \xi_{i}, \varepsilon}$ (resp. $\Pi_{i}^{\perp}=\Pi_{\delta_{i}, \xi_{i}, \varepsilon}^{\perp}$ ) denotes the orthogonal projection $H_{0}^{1}\left(\Omega_{\varepsilon}\right) \rightarrow K_{i}$ (resp. $H_{0}^{1}\left(\Omega_{\varepsilon}\right) \rightarrow K_{i}^{\perp}$ ), then (2.6) can be further rewritten as a system of 4 equations. We have (2.7) and (2.8) for $i=3$ (with $\mu_{j}^{-\frac{1}{p-1}}$ replaced by $c_{j}$ ), together with

$$
\begin{aligned}
\Pi_{1}\left(c_{1} P_{\varepsilon} U_{\delta_{i}, \xi_{i}}+\phi_{1}, c_{2} P_{\varepsilon} U_{\delta_{i}, \xi_{i}}+\phi_{2}\right) & \\
=\Pi_{1}\left(i^{*}\right. & {\left[\mu_{1} f\left(c_{1} P_{\varepsilon} U_{\delta_{1}, \xi_{1}}+\phi_{1}\right)+\sum_{j \neq 1} \beta_{1 j}\left|c_{j} P_{\varepsilon} U_{\delta_{j}, \xi_{j}}+\phi_{j}\right|^{2}\left(c_{1} P_{\varepsilon} U_{\delta_{1}, \xi_{1}}+\phi_{1}\right)\right], } \\
i^{*} & {\left.\left[\mu_{2} f\left(c_{2} P_{\varepsilon} U_{\delta_{1}, \xi_{1}}+\phi_{2}\right)+\sum_{j \neq 2} \beta_{2 j}\left|c_{j} P_{\varepsilon} U_{\delta_{j}, \xi_{j}}+\phi_{j}\right|^{2}\left(c_{2} P_{\varepsilon} U_{\delta_{1}, \xi_{1}}+\phi_{2}\right)\right]\right), }
\end{aligned}
$$

and

$$
\begin{aligned}
& \Pi_{1}^{\perp}\left(c_{1} P_{\varepsilon} U_{\delta_{i}, \xi_{i}}+\phi_{1}, c_{2} P_{\varepsilon} U_{\delta_{i}, \xi_{i}}+\phi_{2}\right) \\
&=\Pi_{1}^{\perp}\left(i^{*}\left[\mu_{1} f\left(c_{1} P_{\varepsilon} U_{\delta_{1}, \xi_{1}}+\phi_{1}\right)+\sum_{j \neq 1} \beta_{1 j}\left|c_{j} P_{\varepsilon} U_{\delta_{j}, \xi_{j}}+\phi_{j}\right|^{2}\left(c_{1} P_{\varepsilon} U_{\delta_{1}, \xi_{1}}+\phi_{1}\right)\right],\right. \\
&\left.i^{*}\left[\mu_{2} f\left(c_{2} P_{\varepsilon} U_{\delta_{1}, \xi_{1}}+\phi_{2}\right)+\sum_{j \neq 2} \beta_{2 j}\left|c_{j} P_{\varepsilon} U_{\delta_{j}, \xi_{j}}+\phi_{j}\right|^{2}\left(c_{2} P_{\varepsilon} U_{\delta_{1}, \xi_{1}}+\phi_{2}\right)\right]\right),
\end{aligned}
$$

5.3. The equations in $\mathbf{K}_{\mathbf{d}, \boldsymbol{\tau}, \varepsilon}^{\perp}$. The equations in $\mathbf{K}_{\mathbf{d}, \boldsymbol{\tau}, \varepsilon}^{\perp}$ can be still expressed in the form

$$
L_{\mathbf{d}, \boldsymbol{\tau}, \varepsilon}^{i}(\phi)=N_{\mathbf{d}, \boldsymbol{\tau}, \varepsilon}^{i}(\phi)+R_{\mathbf{d}, \boldsymbol{\tau}, \varepsilon}^{i} \quad i=1,3,
$$

where for $i=3$ the linear and nonlinear part and the remainder term are defined as in (3.2), (3.3), (3.4), while for $i=1$ we have

$$
\begin{aligned}
& L_{\mathbf{d}, \boldsymbol{\tau}, \varepsilon}^{1}(\phi)= \\
& \Pi_{1}^{\perp}\left(\phi_{1}-i^{*}\left[\mu_{1} f^{\prime}\left(c_{1} P_{\varepsilon} U_{\delta_{1}, \xi_{1}}\right) \phi_{1}+\sum_{j \neq 1} \beta_{1 j}\left(c_{j} P_{\varepsilon} U_{\delta_{j}, \xi_{j}}\right)^{2} \phi_{1}+2 \sum_{j \neq 1} \beta_{1 j}\left(c_{j} P_{\varepsilon} U_{\delta_{j}, \xi_{j}}\right)\left(c_{1} P_{\varepsilon} U_{\delta_{1}, \xi_{1}}\right) \phi_{j}\right],\right. \\
& \left.\phi_{2}-i^{*}\left[\mu_{2} f^{\prime}\left(c_{2} P_{\varepsilon} U_{\delta_{1}, \xi_{1}}\right) \phi_{2}+\sum_{j \neq 2} \beta_{2 j}\left(c_{j} P_{\varepsilon} U_{\delta_{j}, \xi_{j}}\right)^{2} \phi_{2}+2 \sum_{j \neq 2} \beta_{2 j}\left(c_{j} P_{\varepsilon} U_{\delta_{j}, \xi_{j}}\right)\left(c_{2} P_{\varepsilon} U_{\delta_{1}, \xi_{1}}\right) \phi_{j}\right]\right),
\end{aligned}
$$




$$
\begin{aligned}
& N_{\mathbf{d}, \boldsymbol{\tau}, \varepsilon}^{1}(\phi)= \\
& \Pi_{1}^{\perp}\left(i ^ { * } \left[\mu_{1} f\left(c_{1} P_{\varepsilon} U_{\delta_{1}, \xi_{1}}+\phi_{1}\right)-\mu_{1} f\left(c_{1} P_{\varepsilon} U_{\delta_{1}, \xi_{1}}\right)-\mu_{1} f^{\prime}\left(c_{1} P_{\varepsilon} U_{\delta_{1}, \xi_{1}}\right) \phi_{1}\right.\right. \\
& +\sum_{j \neq 1} \beta_{1 j}\left|c_{j} P_{\varepsilon} U_{\delta_{j}, \xi_{j}}+\phi_{j}\right|^{2}\left(c_{1} P_{\varepsilon} U_{\delta_{1}, \xi_{1}}+\phi_{1}\right)-\beta_{1 j}\left(c_{j} P_{\varepsilon} U_{\delta_{j}, \xi_{j}}\right)^{2}\left(c_{1} P_{\varepsilon} U_{\delta_{1}, \xi_{1}}\right) \\
& \left.-\sum_{j \neq 1} \beta_{1 j}\left(c_{j} P_{\varepsilon} U_{\delta_{j}, \xi_{j}}\right)^{2} \phi_{1}-2 \beta_{1 j}\left(c_{j} P_{\varepsilon} U_{\delta_{j}, \xi_{j}}\right)\left(c_{1} P_{\varepsilon} U_{\delta_{1}, \xi_{1}}\right) \phi_{j}\right], \\
& i^{*}\left[\mu_{2} f\left(c_{2} P_{\varepsilon} U_{\delta_{1}, \xi_{1}}+\phi_{2}\right)-\mu_{2} f\left(c_{2} P_{\varepsilon} U_{\delta_{1}, \xi_{1}}\right)-\mu_{2} f^{\prime}\left(c_{2} P_{\varepsilon} U_{\delta_{1}, \xi_{1}}\right) \phi_{2}\right. \\
& +\sum_{j \neq 2} \beta_{2 j}\left|c_{j} P_{\varepsilon} U_{\delta_{j}, \xi_{j}}+\phi_{j}\right|^{2}\left(c_{2} P_{\varepsilon} U_{\delta_{1}, \xi_{1}}+\phi_{2}\right)-\beta_{2 j}\left(c_{j} P_{\varepsilon} U_{\delta_{j}, \xi_{j}}\right)^{2}\left(c_{2} P_{\varepsilon} U_{\delta_{1}, \xi_{1}}\right) \\
& \left.\left.-\sum_{j \neq 2} \beta_{2 j}\left(c_{j} P_{\varepsilon} U_{\delta_{j}, \xi_{j}}\right)^{2} \phi_{2}-2 \beta_{2 j}\left(c_{j} P_{\varepsilon} U_{\delta_{j}, \xi_{j}}\right)\left(c_{2} P_{\varepsilon} U_{\delta_{1}, \xi_{1}}\right) \phi_{j}\right]\right),
\end{aligned}
$$

and

$$
\begin{aligned}
R_{\mathbf{d}, \boldsymbol{\tau}, \varepsilon}^{1}=\Pi_{1}^{\perp}\left(i^{*}\left[c_{1}\left(P_{\varepsilon} U_{\delta_{1}, \xi_{1}}-U_{\delta_{1}, \xi_{1}}\right)+\beta_{13}\left(c_{3} P_{\varepsilon} U_{\delta_{3}, \xi_{3}}\right)^{2}\left(c_{1} P_{\varepsilon} U_{\delta_{1}, \xi_{1}}\right)\right],\right. \\
\left.i^{*}\left[c_{2}\left(P_{\varepsilon} U_{\delta_{1}, \xi_{1}}-U_{\delta_{1}, \xi_{1}}\right)+\beta_{23}\left(c_{3} P_{\varepsilon} U_{\delta_{3}, \xi_{3}}\right)^{2}\left(c_{2} P_{\varepsilon} U_{\delta_{1}, \xi_{1}}\right)\right]\right)
\end{aligned}
$$

where we used the definition of $i^{*}$ and the equations (5.2) defining $c_{1}, c_{2}$.

We define

$$
\mathbf{L}_{\mathbf{d}, \boldsymbol{\tau}, \varepsilon}:=\left(L_{\mathbf{d}, \boldsymbol{\tau}, \varepsilon}^{1}, L_{\mathbf{d}, \boldsymbol{\tau}, \varepsilon}^{3}\right): \mathbf{K}_{\mathbf{d}, \boldsymbol{\tau}, \varepsilon}^{\perp} \rightarrow \mathbf{K}_{\mathbf{d}, \boldsymbol{\tau}, \varepsilon}^{\perp},
$$

and $\mathbf{R}_{\mathbf{d}, \boldsymbol{\tau}, \varepsilon}$ and $\mathbf{N}_{\mathbf{d}, \boldsymbol{\tau}, \varepsilon}$ in an analogue way.

The main result of this subsection is the counterpart of Proposition 3.1 in the present setting.

Proposition 5.3. For every $\eta>0$ small enough there exists $\bar{\beta}, \bar{\varepsilon}>0$ small, and $C>0$, such that if $\varepsilon \in(0, \bar{\varepsilon})$, and $-\infty<\beta_{13}, \beta_{23}<\bar{\beta}$, then for any $(\mathbf{d}, \boldsymbol{\tau}) \in X_{\eta}$ (see (5.7)) there exists a unique function $\boldsymbol{\phi}^{\mathbf{d}, \boldsymbol{\tau}, \varepsilon} \in K_{\mathbf{d}, \boldsymbol{\tau}, \varepsilon}^{\perp}$ solving the equation

$$
\mathbf{L}_{\mathbf{d}, \boldsymbol{\tau}, \varepsilon}(\phi)=\mathbf{R}_{\mathbf{d}, \boldsymbol{\tau}, \varepsilon}+\mathbf{N}_{\mathbf{d}, \boldsymbol{\tau}, \varepsilon}(\phi)
$$

and satisfying

$$
\left\|\phi^{\mathbf{d}, \boldsymbol{\tau}, \varepsilon}\right\|_{H_{0}^{1}\left(\Omega_{\varepsilon}\right)} \leq C \varepsilon
$$

Furthermore, the map $(\varepsilon, \mathbf{d}, \boldsymbol{\tau}) \mapsto \phi^{\mathbf{d}, \boldsymbol{\tau}, \varepsilon}$ is of class $\mathcal{C}^{1}$, and

$$
\left\|\nabla_{(\mathbf{d}, \boldsymbol{\tau})} \phi^{\mathbf{d}, \boldsymbol{\tau}, \varepsilon}\right\|_{H_{0}^{1}\left(\Omega_{\varepsilon}\right)} \leq C \varepsilon
$$

For the proof, we start studying the linear part.

Lemma 5.4. For every $\eta>0$ small enough there exists $\bar{\beta}, \varepsilon_{0}>0$ small, and $C>0$, such that if $\varepsilon \in\left(0, \varepsilon_{0}\right)$, and $-\infty<\beta_{13}, \beta_{23}<\bar{\beta}$, then

$$
\left\|\mathbf{L}_{\mathbf{d}, \boldsymbol{\tau}, \varepsilon}(\boldsymbol{\phi})\right\|_{H_{0}^{1}\left(\Omega_{\varepsilon}\right)} \geq C\|\phi\|_{H_{0}^{1}\left(\Omega_{\varepsilon}\right)} \quad \forall \phi \in H_{0}^{1}\left(\Omega_{\varepsilon}, \mathbb{R}^{3}\right)
$$


for every $(\mathbf{d}, \boldsymbol{\tau}) \in X_{\eta}$. Moreover, $\mathbf{L}_{\mathbf{d}, \boldsymbol{\tau}, \varepsilon}$ is invertible in $\mathbf{K}_{\mathbf{d}, \boldsymbol{\tau}, \varepsilon}^{\perp}$, with continuous inverse.

Proof. The proof follows exactly the same sketch of that of Lemma 3.2. We suppose by contradiction that there exist sequences

$$
\left\{\varepsilon_{n}\right\} \subset \mathbb{R}^{+}, \varepsilon_{n} \rightarrow 0,\left\{\left(\mathbf{d}_{n}, \boldsymbol{\tau}_{n}\right)\right\} \subset X_{\eta},\left\{\phi_{n}=\left(\left(\phi_{1, n}, \phi_{2, n}\right), \phi_{3, n}\right)\right\} \subset K_{1, n}^{\perp} \times K_{3, n}^{\perp}
$$

such that

$$
\left\|\phi_{n}\right\|_{H_{0}^{1}\left(\Omega_{\varepsilon_{n}}\right)}=1 \quad \text { and } \quad\left\|\mathbf{L}_{n}\left(\phi_{n}\right)\right\|_{H_{0}^{1}\left(\Omega_{\varepsilon_{n}}\right)} \rightarrow 0
$$

as $n \rightarrow \infty$, where we adopted the same short notation as in Lemma 3.2.

Let $\mathbf{h}_{n}:=\mathbf{L}_{n}\left(\phi_{n}\right)$. Then we have three equations for $\phi_{1, n}, \phi_{2, n}, \phi_{3, n}$, completely analogue to (3.8) (with $c_{i}$ instead of $\mu_{i}^{-\frac{1}{p-1}}$ ), but with $\left(w_{1, n}, w_{2, n}\right) \in K_{1, n}, w_{3, n} \in K_{3, n}$.

Step 1) $\left\|w_{i, n}\right\|_{H_{0}^{1}\left(\Omega_{n}\right)} \rightarrow 0$ as $n \rightarrow \infty$.

The argument used in Lemma 3.2 immediately implies that $\left\|w_{3, n}\right\|_{H_{0}^{1}\left(\Omega_{n}\right)} \rightarrow 0$. Regarding $w_{1, n}$ and $w_{2, n}$, we multiply the first equation with $\delta_{1, n}^{2} w_{1, n}$, the second equation with $\delta_{1, n}^{2} w_{2, n}$, and we sum the results. Since $\left(\phi_{1, n}, \phi_{2, n}\right),\left(h_{1, n}, h_{2, n}\right) \in K_{1, n}^{\perp}$, and recalling the definition of $\alpha_{i j}$ given in (5.5), we obtain

$$
\begin{aligned}
& \delta_{1, n}^{2}\left(\left\|w_{1, n}\right\|^{2}+\left\|w_{2, n}\right\|^{2}\right)=\int_{\Omega_{n}} c_{3}^{2}\left(P_{n} U_{3, n}\right)^{2}\left(\beta_{13} \phi_{1, n} w_{1, n}+\beta_{23} \phi_{2, n} w_{2, n}\right) \\
& \quad+\int_{\Omega_{n}} c_{3}\left(P_{n} U_{1, n}\right)\left(P_{n} U_{3, n}\right)\left(c_{1} \beta_{13} \phi_{3, n} w_{1, n}+c_{2} \beta_{23} \phi_{3, n} w_{2, n}\right) \\
& \quad+\delta_{1, n}^{2} \int_{\Omega_{n}}\left(P_{n} U_{1, n}\right)^{2}\left(\alpha_{11} \phi_{1, n} w_{1, n}+\alpha_{12}\left(\phi_{2, n} w_{1, n}+\phi_{1, n} w_{2, n}\right)+\alpha_{22} \phi_{2, n} w_{2, n}\right) .
\end{aligned}
$$

In this equation, the first and the second integral on the right hand side can be treated as terms $(I I I)$ and $(I V)$ in (3.9), and together give $o\left(\delta_{1, n}^{2}\right)$ as $n \rightarrow \infty$.

The integral on the left hand side can be treated developed as term $(I)$ in $(3.9)$, in the following way: since $\left(w_{1, n}, w_{2, n}\right) \in K_{1, n}$, there exists constants $c_{1, n}^{h}, h=0, \ldots, 4$ such that

$$
\left(w_{1, n}, w_{2, n}\right)=\sum_{h=0}^{4} c_{1, n}^{h}\left(e_{2},-e_{1}\right) P_{n} \psi_{1, n}^{h}
$$

then, for $\sigma_{l k}$ defined in $(3.11)$, we have

$$
\begin{aligned}
\delta_{1, n}^{2}\left(\left\|w_{1, n}\right\|^{2}+\left\|w_{2, n}\right\|^{2}\right) & =\sum_{l, k=0}^{4} c_{1, n}^{l} c_{1, n}^{k} \underbrace{\left(e_{1}^{2}+e_{2}^{2}\right)}_{=1}\left(\sigma_{l k}+o(1)\right) \\
& =\sum_{h=0}^{4}\left(c_{1, n}^{h}\right)^{2} \sigma_{h h}+o(1) \sum_{h, k=0}^{4} c_{1, n}^{h} c_{1, n}^{k} .
\end{aligned}
$$

It remains then to analyze the last integral on the right hand side in (5.14), and in what follows we prove that it gives

$$
o\left(\delta_{1, n}^{2}\right)\left(\left\|w_{1, n}\right\|+\left\|w_{2, n}\right\|\right)+O\left(\delta_{i, n}^{2}\right) \sum_{l=0}^{N} c_{i, n}^{l} .
$$

Now, since $\left(\phi_{1, n}, \phi_{2, n}\right) \in K_{1, n}^{\perp}$, for every $h$

$$
0=\int_{\Omega_{n}} e_{2} \nabla\left(P_{n} \psi_{1, n}^{h}\right) \cdot \nabla \phi_{1, n}-e_{1} \nabla\left(P_{n} \psi_{1, n}^{h}\right) \cdot \nabla \phi_{2, n}=3 \int_{\Omega_{n}} U_{1, n}^{2} \psi_{1, n}^{h}\left(e_{2} \phi_{1, n}-e_{1} \phi_{2, n}\right) .
$$


Therefore, for every $h$

$$
\begin{aligned}
\int_{\Omega_{n}} U_{1, n}^{2} \psi_{1, n}^{h}\left(\alpha_{11} e_{2} \phi_{1, n}+\alpha_{12}\left(e_{2} \phi_{2, n}-e_{1} \phi_{1, n}\right)-\alpha_{22} e_{1} \phi_{2, n}\right) \\
=\int_{\Omega_{n}} \alpha_{11} U_{1, n}^{2} \psi_{1, n}^{h}\left(e_{2} \phi_{1, n} \pm e_{1} \phi_{2, n}\right)+\int_{\Omega_{n}} \alpha_{12} U_{1, n}^{2} \psi_{1, n}^{h}\left(e_{2} \phi_{2, n}-e_{1} \phi_{1, n}\right) \\
\quad \quad-\int_{\Omega_{n}} \alpha_{22} U_{1, n}^{2} \psi_{1, n}^{h}\left(e_{1} \phi_{2, n} \pm e_{2} \phi_{1, n}\right) \\
=\int_{\Omega_{n}}\left(\alpha_{11} e_{1}+\alpha_{12} e_{2}\right) U_{1, n}^{2} \psi_{1, n}^{h} \phi_{2, n}-\int_{\Omega_{n}}\left(\alpha_{12} e_{1}+\alpha_{22} e_{2}\right) U_{1, n}^{2} \psi_{1, n}^{h} \phi_{1, n} \\
=\lambda_{2} \int_{\Omega_{n}} U_{1, n}^{2} \psi_{1, n}^{h}\left(e_{1} \phi_{2, n}-e_{2} \phi_{1, n}\right)=0,
\end{aligned}
$$

where we used the definition of $\alpha_{i j}$, see (5.5). In turn, using (5.15), we have

$$
\begin{aligned}
& \delta_{1, n}^{2} \int_{\Omega_{n}}\left(P_{n} U_{1, n}\right)^{2}\left(\alpha_{11} \phi_{1, n} w_{1, n}+\alpha_{12}\left(\phi_{2, n} w_{1, n}+\phi_{1, n} w_{2, n}\right)+\alpha_{22} \phi_{2, n} w_{2, n}\right) \\
& \quad=\delta_{1, n}^{2} \int_{\Omega_{n}}\left(\left(P_{n} U_{1, n}\right)^{2}-U_{1, n}\right)^{2}\left(\alpha_{11} \phi_{1, n} w_{1, n}+\alpha_{12}\left(\phi_{2, n} w_{1, n}+\phi_{1, n} w_{2, n}\right)+\alpha_{22} \phi_{2, n} w_{2, n}\right) \\
& \quad+\delta_{1, n}^{2} \sum_{h=0}^{4} c_{1, n}^{h} \int_{\Omega_{n}} U_{1, n}^{2}\left(P_{n} \psi_{1, n}^{h}-\psi_{1, n}^{h}\right)\left(\alpha_{11} e_{2} \phi_{1, n}+\alpha_{12}\left(e_{2} \phi_{2, n}-e_{1} \phi_{1, n}\right)-\alpha_{22} e_{1} \phi_{2, n}\right),
\end{aligned}
$$

and hence we can proceed exactly as in (B.3), deducing that

$$
\begin{aligned}
\delta_{1, n}^{2} \int_{\Omega_{n}}\left(P_{n} U_{1, n}\right)^{2}\left(\alpha_{11} \phi_{1, n} w_{1, n}+\alpha_{12}\left(\phi_{2, n} w_{1, n}\right.\right. & \left.\left.+\phi_{1, n} w_{2, n}\right)+\alpha_{22} \phi_{2, n} w_{2, n}\right) \\
& =o\left(\delta_{1, n}^{2}\right)\left(\left\|w_{1, n}\right\|+\left\|w_{2, n}\right\|\right)+O\left(\delta_{i, n}^{2}\right) \sum_{l=0}^{N} c_{i, n}^{l} .
\end{aligned}
$$

To sum up, we proved the analogue of the estimates (3.10) for the couple $\left(w_{1, n}, w_{2, n}\right)$, and hence we can conclude as in Lemma 3.2 that $\left\|w_{1, n}\right\|,\left\|w_{2, n}\right\| \rightarrow 0$ as $n \rightarrow \infty$.

Step 2) For a fixed $\kappa=1, \ldots, m$, we introduce

$$
\tilde{\phi}_{i, n}^{\kappa}(y):=\left\{\begin{array}{ll}
\delta_{\kappa, n}^{\frac{N-2}{2}} \phi_{i, n}\left(\xi_{\kappa, n}+\delta_{\kappa, n} y\right) & y \in \frac{\Omega_{n}-\xi_{\kappa, n}}{\delta_{\kappa, n}}=: \tilde{\Omega}_{\kappa, n} \\
0 & y \in \mathbb{R}^{N} \backslash \tilde{\Omega}_{\kappa, n},
\end{array} \quad i=1, \ldots, m .\right.
$$

In a completely analogue way, we define $\tilde{h}_{i, n}^{\kappa}$ and $\tilde{w}_{i, n}^{\kappa}$. Proceeding as in step 2 of Lemma 3.2 (with minor changes), it is not difficult to check that $\tilde{\phi}_{1, n}^{1}, \tilde{\phi}_{2, n}^{1}, \tilde{\phi}_{3, n}^{3} \rightarrow 0$ in $\mathcal{D}^{1,2}\left(\mathbb{R}^{N}\right)$ as $n \rightarrow \infty$. The only difference with respect to Lemma 3.2 is that this time the weak limit $\left(\tilde{\phi}_{1}^{1}, \tilde{\phi}_{2}^{1}\right)$ solves the system (5.4) instead of a single equation, and hence the fact that $\left(\tilde{\phi}_{1}^{1}, \tilde{\phi}_{2}^{1}\right)=(0,0)$ comes from Proposition 5.2 and the condition $\left(\phi_{1, n}, \phi_{2, n}\right) \in K_{1, n}^{\perp}$.

Step 3) We prove that $\left\|\phi_{i, n}\right\|_{H_{0}^{1}\left(\Omega_{n}\right)} \rightarrow 0$ as $n \rightarrow \infty$ for every $i$. Regarding $\phi_{3, n}$, we can proceed as in Lemma 3.2. We focus then on the other components. We test (3.8) with $\phi_{1, n}$ : recalling that 
$\left\{\phi_{1, n}\right\}$ is bounded in $H_{0}^{1}\left(\Omega_{n}\right)$ and that $w_{1, n}, h_{1, n} \rightarrow 0$ strongly, we deduce that

$$
\begin{aligned}
\left\|\phi_{1, n}\right\|_{H_{0}^{1}\left(\Omega_{n}\right)}^{2} & =o(1)+\int_{\Omega_{n}}\left(P_{n} U_{i, n}\right)^{2}\left(\alpha_{11} \phi_{1, n}^{2}+\alpha_{12} \phi_{1, n} \phi_{2, n}\right) \\
& +\beta_{13} \int_{\Omega_{n}}\left(c_{3} P_{n} U_{3, n}\right)^{2} \phi_{1, n}^{2}+2 \beta_{23} \int_{\Omega_{n}}\left(c_{1} P_{n} U_{i, n}\right)\left(c_{3} P_{n} U_{3, n}\right) \phi_{1, n} \phi_{3, n}
\end{aligned}
$$

The second and the third integral on the right hand side can be treated as in Lemma 3.2. Notice that here we have only to ask that $\beta_{13}$ is small enough (we don't need any assumption on $\beta_{12}$ ).

Now, to estimate the first integral in (5.16), we observe that as in (3.17)

$$
\int_{\Omega_{n}}\left(P_{n} U_{1, n}\right)^{2} \phi_{1, n}^{2} \rightarrow 0
$$

and moreover

$$
\begin{aligned}
\left|\int_{\Omega_{n}}\left(P_{n} U_{1, n}\right)^{2} \phi_{1, n} \phi_{2, n}\right| & \leq C \int_{\Omega_{n}} U_{1, n}^{2}\left|\phi_{1, n}\right|\left|\phi_{2, n}\right| \\
& =C \int_{\tilde{\Omega}_{1, n}} U_{1,0}^{2} \tilde{\phi}_{1, n}^{1} \tilde{\phi}_{2, n}^{1} \rightarrow 0
\end{aligned}
$$

as $n \rightarrow \infty$, since $\tilde{\phi}_{1, n}^{1}, \tilde{\phi}_{2, n}^{1} \rightarrow 0$ in $L^{\frac{2 N}{N-2}}\left(\mathbb{R}^{N}\right)$ by step 2 , and $U_{1,0}^{2} \in L^{\frac{N}{2}}\left(\mathbb{R}^{N}\right)$. Thus, we infer that $\left\|\phi_{1, n}\right\| \rightarrow 0$; in the same way, $\left\|\phi_{2, n}\right\| \rightarrow 0$, and we reached the desired contradiction.

We stress that, while we have to suppose $-\infty<\beta_{13}, \beta_{23}<\bar{\beta}$, no assumption is needed on $\beta_{12}$. Step 4) Invertibility of $\mathbf{L}_{\mathbf{d}, \boldsymbol{\tau}, \varepsilon}$. This can be proved exactly as in Lemma 3.2.

The rest of the proof of Proposition 5.3 is now a straightforward modification of that of Proposition 3.1, and hence is omitted.

5.4. The reduced problem. In this section we solve equation (5.8) with $\phi=\phi^{\mathrm{d}, \boldsymbol{\tau}, \varepsilon}$. In what follows we use the notation

$$
V_{i}^{\mathbf{d}, \boldsymbol{\tau}, \varepsilon}:=c_{i} P_{\varepsilon} U_{\delta_{1}, \xi_{1}}^{\mathbf{d}, \boldsymbol{\tau}, \varepsilon}+\phi_{i}^{\mathbf{d}, \boldsymbol{\tau}, \varepsilon} \quad i=1,2, \quad V_{3}^{\mathbf{d}, \boldsymbol{\tau}, \varepsilon}:=c_{3} P_{\varepsilon} U_{\delta_{3}, \xi_{3}}^{\mathbf{d}, \boldsymbol{\tau}, \varepsilon}+\phi_{3}^{\mathbf{d}, \boldsymbol{\tau}, \varepsilon} .
$$

Let $J_{\varepsilon}: H_{0}^{1}\left(\Omega_{\varepsilon}, \mathbb{R}^{3}\right) \rightarrow \mathbb{R}$ be the action functional, defined as in (4.1). Critical points of $J_{\varepsilon}$ are solution to (2.1), and hence solutions to (1.4).

Let us introduce now the reduced functional $\tilde{J}_{\varepsilon}: X_{\eta} \rightarrow \mathbb{R}$,

$$
\tilde{J}_{\varepsilon}(\mathbf{d}, \boldsymbol{\tau}):=J_{\varepsilon}\left(c_{1} P_{\varepsilon} U_{\delta_{1}, \xi_{1}}+\phi_{1}^{\mathbf{d}, \boldsymbol{\tau}, \varepsilon}, c_{2} P_{\varepsilon} U_{\delta_{1}, \xi_{1}}+\phi_{2}^{\mathbf{d}, \boldsymbol{\tau}, \varepsilon}, c_{3} P_{\varepsilon} U_{\delta_{3}, \xi_{3}}+\phi_{3}^{\mathbf{d}, \boldsymbol{\tau}, \varepsilon}\right) .
$$

The counterpart of Lemma 4.1 in the present context is given in the following statement, whose proof is omitted.

Lemma 5.5. There exists $\bar{\varepsilon}>0$ sufficiently small such that if $(\mathbf{d}, \boldsymbol{\tau})=\left(d_{1}, d_{3}, \tau_{1}, \tau_{3}\right)$ is a critical point of $\tilde{J}_{\varepsilon}$, and $\varepsilon \in(0, \bar{\varepsilon})$, then

$$
\left(c_{1} P_{\varepsilon} U_{\delta_{1}, \xi_{1}}+\phi_{1}^{\mathbf{d}, \boldsymbol{\tau}, \varepsilon}, c_{2} P_{\varepsilon} U_{\delta_{1}, \xi_{1}}+\phi_{2}^{\mathbf{d}, \boldsymbol{\tau}, \varepsilon}, c_{3} P_{\varepsilon} U_{\delta_{3}, \xi_{3}}+\phi_{3}^{\mathbf{d}, \boldsymbol{\tau}, \varepsilon}\right)
$$

is a solution to (2.7), and hence a solution to (2.1).

Due to Lemma 5.5, we have to find a critical point of the reduced functional $\tilde{J}_{\varepsilon}$ in $X_{\eta}$, and to this aim we derive the asymptotic expansion of $J_{\varepsilon}\left(V_{1}^{\mathbf{d}, \boldsymbol{\tau}, \varepsilon}, V_{2}^{\mathbf{d}, \boldsymbol{\tau}, \varepsilon}, V_{3}^{\mathbf{d}, \boldsymbol{\tau}, \varepsilon}\right)$ as $\varepsilon \rightarrow 0^{+}$. We recall 
the definition of $b_{1}, b_{2}$ and $\Gamma$, given in (4.6) and (4.7), but we modify the definition of $\Psi$ in the following way:

$$
\begin{aligned}
\Psi(\mathbf{d}, \boldsymbol{\tau}) & :=\left(c_{1}^{2}+c_{2}^{2}\right)\left[b_{2} H\left(a_{1}, a_{1}\right) d_{1}^{N-2}+\frac{\alpha_{N}^{p+1} r_{1}^{N-2}}{2} \frac{\Gamma\left(\tau_{1}\right)}{d_{1}^{N-2}\left(1+\left|\tau_{1}\right|^{2}\right)^{\frac{N-2}{2}}}\right] \\
& +c_{3}^{2}\left[b_{2} H\left(a_{1}, a_{1}\right) d_{1}^{N-2}+\frac{\alpha_{N}^{p+1} r_{1}^{N-2}}{2} \frac{\Gamma\left(\tau_{1}\right)}{d_{1}^{N-2}\left(1+\left|\tau_{1}\right|^{2}\right)^{\frac{N-2}{2}}}\right] .
\end{aligned}
$$

Proposition 5.6. We have

$$
\tilde{J}_{\varepsilon}(\mathbf{d}, \boldsymbol{\tau})=\left(\sum_{i=1}^{3} c_{i}^{2}\right) b_{1}+\Psi(\mathbf{d}, \boldsymbol{\tau}) \varepsilon^{\frac{N-2}{2}}+R_{\varepsilon}(\mathbf{d}, \boldsymbol{\tau}),
$$

with $R_{\varepsilon}=o\left(\varepsilon^{\frac{N-2}{2}}\right) \mathcal{C}^{1}$-uniformly in $X_{\eta}$ as $\varepsilon \rightarrow 0$.

Proof. In this proof we omit the dependence of the functions on $\mathbf{d}, \boldsymbol{\tau}, \varepsilon$ for simplicity, and we write $U_{1}=U_{2}=U_{\delta_{1}, \xi_{1}}, U_{3}=U_{\delta_{3}, \xi_{3}}$.

We proceed as in Proposition 4.2, noting that by (5.2)

$$
\begin{aligned}
\tilde{J}_{\varepsilon}(\mathbf{d}, \boldsymbol{\tau}) & =\sum_{i} I_{\varepsilon}\left(V_{i}\right)-\frac{2}{p+1} \sum_{i<j} \int_{\Omega_{\varepsilon}} \beta_{i j}\left|V_{i}\right|^{\frac{p+1}{2}}\left|V_{j}\right|^{\frac{p+1}{2}} \\
& =\sum_{i} I_{\varepsilon}\left(c_{i} P U_{i}\right)+\frac{1}{2} \sum_{i} \int_{\Omega_{\varepsilon}} 2 c_{i} \nabla\left(P U_{i}\right) \cdot \nabla \phi_{i}+\left|\nabla \phi_{i}\right|^{2} \\
& -\sum_{i} \int_{\Omega_{\varepsilon}} \mu_{i}\left(F\left(c_{i} P U_{i}+\phi_{i}\right)-F\left(c_{i} P U_{i}\right)\right) \\
& -\frac{2}{p+1} \sum_{i<j} \int_{\Omega_{\varepsilon}}\left|c_{i} P U_{i}\right|^{2}\left|c_{j} P U_{j}\right|^{2} \\
& -\sum_{i<j} \int_{\Omega_{\varepsilon}}\left(\left|c_{i} P U_{i}+\eta_{i} \phi_{i}\right|^{2}\left|c_{j} P U_{j}+\eta_{j} \phi_{j}\right| \phi_{j}\right. \\
& \left.\quad+\left|c_{i} P U_{i}+\eta_{i} \phi_{i}\right|\left|c_{j} P U_{j}+\eta_{j} \phi_{j}\right|^{2} \phi_{i}\right) \\
= & \left(c_{1}^{2}+c_{2}^{2}\right) I_{\varepsilon}\left(P U_{1}\right)+c_{3}^{2} I_{\varepsilon}\left(P U_{3}\right)+R_{1, \varepsilon}
\end{aligned}
$$

(where $\eta_{i}, \eta_{j}$ denote continuous functions with values in $[0,1]$ ). Then we can slightly modify Lemmas 4.3 and 4.4 to obtain the desired result.

Once that Proposition 5.6 is established, the conclusion of the proof of Theorem 1.2 is analogue to the one of Theorem 1.1.

\section{Proof of Proposition 1.4}

Let us consider a system

$$
-\Delta U_{i}=\sum_{j=1}^{k} \beta_{i j} U_{i} U_{j}^{2} \quad \text { in } \mathbb{R}^{4}, i=1, \ldots, k,
$$


and let us suppose that it has a solution $U_{i}=c_{i} U$, with $c_{i}>0$ for $i=1, \ldots, k$, where $U=U_{1,0}$ is a standard bubble in $\mathbb{R}^{4}$ centered in 0 and with $\delta=1$. That is,

$$
\sum_{j=1, \ldots, k} \beta_{i j} c_{j}^{2}=1, \quad i=1, \ldots, k .
$$

We can linearize system $(6.1)$ in $\mathcal{D}^{1,2}\left(\mathbb{R}^{N}\right)$ around the solutions $\left(c_{1} U, \ldots, c_{k} U\right)$, obtaining

$$
-\Delta v_{i}=[\underbrace{\left(3 \beta_{i i} c_{i}^{2}+\sum_{j \neq i} \beta_{i j} c_{j}^{2}\right)}_{=1+2 \beta_{i i} c_{i}^{2}} v_{i}+2 \sum_{j \neq i} \beta_{i j} c_{i} c_{j} v_{j}] U^{2} \quad \text { in } \mathbb{R}^{4},
$$

with $v_{i} \in \mathcal{D}^{1,2}\left(\mathbb{R}^{4}\right)$.

It is clear that Proposition 1.4 follows if we prove that the condition

the matrix $\left(\beta_{i j}\right)_{i, j=1, \ldots, k}$ is invertible and has only positive entries

implies that (6.2) has a 5-dimensional set of solutions.

To this aim, we observe that system (6.2) can be rewritten as

$$
-\Delta \mathbf{v}=U^{2} \mathcal{M} \mathbf{v} \text { in } \mathbb{R}^{4}, \quad \mathbf{v}:=\left(v_{1}, \ldots, v_{k}\right) \in \mathcal{D}^{1,2}\left(\mathbb{R}^{4}, \mathbb{R}^{k}\right),
$$

with $\mathcal{M}:=\mathcal{I} d+2 \mathcal{C}$ and

$$
\mathcal{C}:=\left(\begin{array}{cccc}
\beta_{11} c_{1}^{2} & \beta_{12} c_{1} c_{2} & \ldots & \beta_{1 k} c_{1} c_{k} \\
\beta_{12} c_{1} c_{2} & \beta_{22} c_{2}^{2} & \ldots & \beta_{2 k} c_{2} c_{k} \\
\vdots & \vdots & \ddots & \vdots \\
\beta_{1 k} c_{1} c_{k} & \beta_{2 k} c_{2} c_{k} & \ldots & \beta_{k k} c_{k}^{2}
\end{array}\right) .
$$

Let $\Lambda$ be an eigenvalue of $\mathcal{M}$ and $e$ an associated eigenfunction, i.e.

$$
\mathcal{M} e=\Lambda e .
$$

It is useful to point out that $\Lambda_{\ell}$ is an eigenvalue of $\mathcal{M}$ if and only if $\Theta_{\ell}:=\left(\Lambda_{\ell}-1\right) / 2$ is an eigenvalue of the matrix $\mathcal{C}$. It is immediate to check that $\Theta=1$ is an eigenvalue of $\mathcal{C}$ whose eigenvector is $\left(c_{1}, \ldots, c_{k}\right)$. We set $\Theta_{1}=1$, which implies $\Lambda_{1}=3$.

Let us consider the eigenvalue problem

$$
-\Delta v=\nu U^{2} v, \quad v \in \mathcal{D}^{1,2}\left(\mathbb{R}^{4}\right) .
$$

It is well known (see [4, Lemma A.1]) that there exists a sequence of positive eigenvalues $\left\{\nu_{m}\right\}_{m \in \mathbb{N}}$ with

$$
1=\nu_{1}<3=\nu_{2}<\nu_{3}<\cdots<\nu_{m}<\nu_{m+1}<\ldots \quad \text { and } \quad \nu_{m} \rightarrow+\infty
$$

The role of these eigenvalues when dealing with (6.2) is clarified by the following statement.

Lemma 6.1. Assume that, for any $\Lambda_{2}, \ldots, \Lambda_{k}$ of $\mathcal{M}$ do not coincide with any of the eigenvalues $\left\{\nu_{m}: m \in \mathbb{N}\right\}$. Then the set of solutions to the linear system (6.2) is 5-dimensional, and is generated by

$$
\psi_{1,0}^{h} \mathfrak{e}_{1} \quad h=0,1, \ldots, 4
$$

where $\mathfrak{e}_{1} \in \mathbb{R}^{k}$ is an eigenvector associated with $\Lambda_{1}=3$ and the functions $\psi_{1,0}^{h}$ have been defined in (1.14). 
Proof. Let $\Lambda_{\ell}$ be an eigenvalue of the matrix $\mathcal{M}$ and let $\mathfrak{e}_{\ell} \in \mathbb{R}^{k}$ an associated eigenvector. We multiply (6.2) by $\mathfrak{e}_{\ell}$ and taking into account the symmetry of the matrix $\mathcal{M}$ we get

$$
-\Delta\left(\mathfrak{e}_{\ell} \cdot \mathbf{v}\right)=\Lambda_{\ell} U^{2}\left(\mathfrak{e}_{\ell} \cdot \mathbf{v}\right) \text { in } \mathbb{R}^{4} .
$$

Since $\Lambda_{\ell} \neq \nu_{m}$ for every $m$, we deduce that

$$
\mathfrak{e}_{\ell} \cdot \mathbf{v}=0 \text { for any } \ell=2, \ldots, k,
$$

which implies (by the orthogonality of eigenvectors associated to different eigenvalues) that

$$
\mathbf{v}=\psi(x) \mathfrak{e}_{1} \text { for some function } \psi \text { such that }-\Delta \psi=3 U^{2} \psi \text { in } \mathbb{R}^{4} .
$$

The claim follows then by [4, Lemma A.1].

Conclusion of the proof of Proposition 1.4. As observed above, we have to prove that if $\left(\beta_{i j}\right)$ is invertible and has positive entries, then the set of solutions to (6.2) is 5-dimensional. By Lemma 6.1 , this amounts to show that if $\left(\beta_{i j}\right)$ is invertible and has positive entries, then the eigenvalues $\Lambda_{2}, \ldots, \Lambda_{k}$ of $\mathcal{M}$ are different from $\nu_{1}=1, \nu_{2}=3, \nu_{m}>3$.

Let us argue in terms of the matrix $\mathcal{C}$. By assumption, $\mathcal{C}$ has positive entries. Therefore by Perron-Frobenius Theorem we deduce that the eigenvalue $\Theta_{1}=1$, which is associated to the eigenvector of positive elements $\left(c_{1}, \ldots, c_{k}\right)$, is simple, and any other eigenvalue $\Theta_{\ell}$ satisfies $\left|\Theta_{\ell}\right|<1$. Moreover, 0 is not an eigenvalue of the matrix $\mathcal{C}$, since a straightforward computation shows that

$$
\operatorname{det} \mathcal{C}=-\left(c_{1}^{2} \cdots c_{k}^{2}\right) \operatorname{det}\left(\beta_{i j}\right) \neq 0
$$

being $\left(\beta_{i j}\right)$ invertible. Therefore, $\Lambda_{1}=3$ is a simple eigenvalue, and we have that both $-1<\Lambda_{\ell}<3$ and $\Lambda_{\ell} \neq 1$ for any $\ell=2, \ldots, k$. This completes the proof.

\section{ApPEndix A.}

In this section we collect several technical lemmas. We start recalling from [14, Lemma 3.1] the point-wise estimate of the difference of $U_{\delta, \xi}$ and $P_{\varepsilon} U_{\delta, \xi}$, as well as of their derivates.

Lemma A.1. Let $a \in \Omega, r>0$, and let $\eta, \varepsilon>0$ be small. Let $d>0$ and $\tau \in \mathbb{R}^{N}$ be such that $\eta<d<\eta^{-1},|\tau|<\eta^{-1}$. Finally, let $\delta=d \sqrt{\varepsilon}$ and $\xi=a+\delta \tau$. Let us define

$$
R(x):=P_{\varepsilon} U_{\delta, \xi}(x)-U_{\delta, \xi}(x)+\alpha_{N} \delta^{\frac{N-2}{2}} H(x, \xi)+\alpha_{N} \frac{1}{\delta^{\frac{N-2}{2}}\left(1+|\tau|^{2}\right)^{\frac{N-2}{2}}}\left(\frac{r \varepsilon}{|x-a|}\right)^{N-2} .
$$

Then there exists a positive constant $C>0$ depending only on $\eta$ and on $\operatorname{dist}(a, \partial \Omega)$ such that for any $x \in \Omega \backslash B_{r \varepsilon}(a)$

$$
\begin{aligned}
|R(x)| & \leq C \delta^{\frac{N-2}{2}}\left[\frac{\varepsilon^{N-2}\left(1+\varepsilon \delta^{1-N}\right)}{|x-a|^{N-2}}+\delta^{2}+\left(\frac{\varepsilon}{\delta}\right)^{N-2}\right] \\
\left|\partial_{\tau_{i}} R(x)\right| & \leq C \delta^{\frac{N}{2}}\left[\frac{\varepsilon^{N-2}\left(1+\varepsilon \delta^{-N}\right)}{|x-a|^{N-2}}+\delta^{2}+\frac{\varepsilon^{N-2}}{\delta^{N-1}}\right] \\
\left|\partial_{\delta} R(x)\right| & \leq C \delta^{\frac{N-4}{2}}\left[\frac{\varepsilon^{N-2}\left(1+\varepsilon \delta^{1-N}\right)}{|x-a|^{N-2}}+\delta^{2}+\left(\frac{\varepsilon}{\delta}\right)^{N-2}\right] .
\end{aligned}
$$

Corollary A.2. In the previous setting, we have

$$
\partial_{\xi_{h}}\left(P_{\varepsilon} U_{\delta, \xi}(x)-U_{\delta, \xi}(x)\right)=-\alpha_{N} \delta^{\frac{N-2}{2}} \partial_{\xi_{h}} H(x, \xi)+\frac{\alpha_{N}(N-2) \tau_{h}}{\delta^{\frac{N}{2}}\left(1+|\tau|^{2}\right)^{\frac{N}{2}}}\left(\frac{r \varepsilon}{|x-a|}\right)^{N-2}+\frac{1}{\delta} \partial_{\tau_{h}} R(x),
$$


and

$$
\begin{aligned}
\partial_{\delta}\left(P_{\varepsilon} U_{\delta, \xi}(x)-U_{\delta, \xi}(x)\right)=-\alpha_{N} \frac{N-2}{2} \delta^{\frac{N-4}{2}} H(x, \xi) & \\
& +\frac{\alpha_{N}(N-2)}{2 \delta^{\frac{N}{2}}\left(1+|\tau|^{2}\right)^{\frac{N-2}{2}}}\left(\frac{r \varepsilon}{|x-a|}\right)^{N-2}+\partial_{\delta} R(x),
\end{aligned}
$$

and in particular there exists $C>0$ depending only on $\eta$ and on $\operatorname{dist}(a, \partial \Omega)$ such that

$$
\begin{gathered}
\left|P_{\varepsilon} U_{\delta, \xi}(x)-U_{\delta, \xi}(x)\right| \leq C \delta^{\frac{N-2}{2}}+C \frac{\delta^{\frac{3}{2} N-3}}{|x-a|^{N-2}} \\
\left|P_{\varepsilon} \psi_{i}^{h}(x)-\psi_{i}^{h}(x)\right| \leq C \delta^{\frac{N-2}{2}}+C \frac{\delta^{\frac{3}{2} N-4}}{|x-a|^{N-2}} \\
\left|P_{\varepsilon} \psi_{i}^{0}(x)-\psi_{i}^{0}(x)\right| \leq C \delta^{\frac{N-4}{2}}+C \frac{\delta^{\frac{3}{2} N-4}}{|x-a|^{N-2}},
\end{gathered}
$$

for $h=1, \ldots, N$, for every $\varepsilon>0$ small enough. In particular, if we fix a compact set $K \Subset \Omega$, then one can choose a unique constant $C>0$ for any $a \in K$.

Proof. The result is a straightforward consequence of Lemma A.1 and of the boundedness of $H(x, \xi)$ together with its derivatives for $x \in \Omega, \xi \in K^{\prime}$, with $K^{\prime}$ compact of $\Omega$. We also used the facts that $\delta \simeq \varepsilon^{\frac{1}{2}}$ and that the derivatives commute with the projection $P_{\varepsilon}$.

Now we collect some estimates regarding the derivates of the bubble.

Lemma A.3. Let $\psi_{\delta, \xi}^{\ell}(\ell=0, \ldots, N)$ be defined in (1.14). Then we have

$$
\begin{aligned}
\text { (i) } \quad\left|\psi_{\delta, \xi}^{0}\right| & \leq \frac{C}{\delta} U_{\delta, \xi} \\
\text { (ii) } \quad\left|\psi_{\delta, \xi}^{\ell}\right| & \leq \frac{C}{\delta} U_{\delta, \xi}^{\frac{N}{N-2}}\left|x_{\ell}-\xi_{\ell}\right| \quad \ell=1, \ldots, N
\end{aligned}
$$

point-wisely in $\mathbb{R}^{N}$.

Proof. The thesis is a simple consequence of the explicit expressions.

The following lemma is an easy consequence of Taylor expansion.

Lemma A.4. For every $q>1$, there exists $C>0$ such that

$$
|| a+\left.b\right|^{q}-|a|^{q} \mid \leq C\left(|a|^{q-1}|b|+|b|^{q}\right) \quad \text { for every } a, b \in \mathbb{R} .
$$

Let also $f(s):=\left(s^{+}\right)^{q}$, with $q \geq 2$. Then there exists $C>0$ such that

$$
\begin{aligned}
\mid f\left(a+b_{1}\right)-f(a)-f^{\prime}(a) b_{1} & -\left(f\left(a+b_{2}\right)-f(a)-f^{\prime}(a) b_{2}\right) \mid \\
\leq & C|| a|+| b_{1}|+| b_{2}||^{q-2}\left(\left|b_{1}\right|+\left|b_{2}\right|\right)\left|b_{1}-b_{2}\right|
\end{aligned}
$$

for every $a, b_{1}, b_{2} \in \mathbb{R}$.

A slightly more involved result is contained in the following statement.

Lemma A.5. Let $N=3,4, p=(N+2) /(N-2)$,

$$
F(x, y):=|x|^{\frac{p-3}{2}} x|y|^{\frac{p+1}{2}},
$$

and

$$
\psi(h, k):=F(x+h, y+k)-F(x, y)-\partial_{1} F(x, y) h-\partial_{2} F(x, y) k .
$$


Then $F, \psi \in \mathcal{C}^{1,1}\left(\mathbb{R}^{2}\right)$, and

$$
\begin{aligned}
& \mid \psi\left(h_{1}, k_{1}\right)-\psi\left(h_{2}, k_{2}\right) \mid \\
& \leq C\left[\frac{p-3}{2}|| x|+| h_{1}|+| h_{2}||^{\frac{p-5}{2}}|| y|+| k_{1}|+| k_{2}||^{\frac{p+1}{2}}|| h_{1}|+| h_{2}||\left|h_{1}-h_{2}\right|\right. \\
& \quad+\left.|| x|+| h_{1}|+| h_{2}\right|^{\frac{p-3}{2}}|| y|+| k_{1}|+| k_{2}||^{\frac{p-1}{2}}|| k_{1}|+| k_{2}||\left|h_{1}-h_{2}\right| \\
& \quad+|| x|+| h_{1}|+| h_{2}||^{\frac{p-3}{2}}|| y|+| k_{1}|+| k_{2}||^{\frac{p-1}{2}}|| h_{1}|+| h_{2}||\left|k_{1}-k_{2}\right| \\
&\left.\quad+|| x|+| h_{1}|+| h_{2}||^{\frac{p-1}{2}}|| y|+| k_{1}|+| k_{2}||^{\frac{p-3}{2}}|| k_{1}|+| k_{2}||\left|k_{1}-k_{2}\right|\right]
\end{aligned}
$$

for every $x, y, h_{1}, k_{1}, h_{2}, k_{2} \in \mathbb{R}$.

Proof. By the Lagrange intermediate value theorem

$$
\left|\psi\left(h_{1}, k_{1}\right)-\psi\left(h_{2}, k_{2}\right)\right|=\left|\nabla \psi(\bar{h}, \bar{k}) \cdot\left(h_{1}-h_{2}, k_{1}-k_{2}\right)\right|,
$$

for some $(\bar{h}, \bar{k})$ on the segment joining $\left(h_{1}, k_{1}\right)$ with $\left(h_{2}, k_{2}\right)$. By definition, and using again the Lagrange theorem,

$$
\begin{aligned}
\left|\partial_{1} \psi(\bar{h}, \bar{k})\right| & =\left|\partial_{1} F(x+\bar{h}, y+\bar{k})-\partial_{1} F(x, y)\right| \\
& \leq\left|\partial_{11} F(x+\tilde{h}, y+\tilde{k}) \bar{h}\right|+\left|\partial_{12} F(x+\tilde{h}, y+\tilde{k}) \bar{k}\right|,
\end{aligned}
$$

where $(\tilde{h}, \tilde{k})$ is on the segment joining $(0,0)$ and $(\bar{h}, \bar{k})$. A similar estimate holds for $\left|\partial_{2} \psi(\bar{h}, \bar{k})\right|$. Observing that

$$
\begin{array}{rlrl} 
& |\bar{h}| \leq\left|h_{1}\right|+\left|h_{2}\right| & \text { and } & |\bar{k}| \leq\left|k_{1}\right|+\left|k_{2}\right|, \\
\Longrightarrow & |\tilde{h}| \leq\left|h_{1}\right|+\left|h_{2}\right| \text { and } & |\tilde{k}| \leq\left|k_{1}\right|+\left|k_{2}\right|,
\end{array}
$$

and using the explicit expression of $F$, the thesis follows.

Now we collect several estimates regarding integrals of the bubbles.

Lemma A.6. Let $K \subset \subset \Omega$. Then, as $\delta \rightarrow 0^{+}$, we have

$$
\int_{\Omega} U_{\delta, \xi}^{q}= \begin{cases}O\left(\delta^{\frac{N-2}{2} q}\right) & \text { if } 0<q<\frac{N}{N-2} \\ C \delta^{\frac{N}{2}}|\log \delta|+O\left(\delta^{\frac{N}{2}}\right) & \text { if } q=\frac{N}{N-2} \\ O\left(\delta^{N-\frac{N-2}{2} q}\right) & \text { if } \frac{N}{N-2}<q<+\infty, q \neq \frac{2 N}{N-2} \\ C & q=\frac{2 N}{N-2}\end{cases}
$$

uniformly in $\xi \in K$, where $C>0$ denotes a constant depending only on the dimension $N$.

Proof. We focus on the cases $q \neq N /(N-2), 2 N /(N-2)$ (if equality holds, one can proceed in the same way). Since $\Omega$ is bounded, there exists $R>0$ such that $\Omega \subset B_{R}(\xi)$. Then

$$
\int_{\Omega} U_{\delta, \xi}^{q} \leq \int_{B_{R}(\xi)} U_{\delta, \xi}^{q}=\alpha_{N}^{q} \int_{B_{R / \delta}} \delta^{N-\frac{N-2}{2} q} \frac{d y}{\left(1+|y|^{2}\right)^{\frac{N-2}{2} q}}
$$

Now, if $q>N /(N-2)$ the last integral can be controlled with the one over $\mathbb{R}^{N}$, which is convergent, and the thesis follows. If on the other hand $q<N /(N-2)$, we have

$$
\begin{aligned}
\int_{\Omega} U_{\delta, \xi}^{q} & \leq C \delta^{N-\frac{N-2}{2} q}\left(1+\int_{1}^{R / \delta} r^{N-1-(N-2) q} d r\right) \\
& \leq C \delta^{N-\frac{N-2}{2} q} \cdot \frac{C}{\delta^{N-(N-2) q}} \leq C \delta^{\frac{N-2}{2} q}
\end{aligned}
$$


In a similar way:

Lemma A.7. Let $r>0, \eta \in(0,1), a \in K \subset \subset \Omega,|\tau|<\eta^{-1}, \xi=a+\delta \tau, 0 \leq \nu_{2}<N, \nu_{1} \geq 0$, $h=1, \ldots, N$, and let $(N-2) q+\nu_{2}-\nu_{1}>N$. Then, as $\delta \rightarrow 0^{+}$, we have

$$
\int_{\Omega \backslash B_{r \delta^{2}}(a)} U_{\delta, \xi}^{q} \frac{\left|x_{h}-\xi_{h}\right|^{\nu_{1}}}{|x-a|^{\nu_{2}}}=O\left(\delta^{N+\nu_{1}-\nu_{2}-\frac{N-2}{2} q}\right),
$$

uniformly in $a \in K$ and $|\tau|<\eta^{-1}$.

If $\nu_{2}=0$ and $q=\left(N+\nu_{1}\right) /(N-2)$, then

$$
\int_{\Omega} U_{\delta, \xi}^{q}|x-\xi|^{\nu_{1}}=O\left(\delta^{\frac{N-2}{2} q}|\log \delta|\right)
$$

as $\delta \rightarrow 0^{+}$, uniformly in $a \in K$ and $|\tau|<\eta^{-1}$.

If $\nu_{1}=0, \nu_{2}=N$, and $(N-2) q+\nu_{2}>N$, then

$$
\int_{\Omega \backslash B_{r \delta^{2}}(a)} \frac{U_{\delta, \xi}^{q}}{|x-a|^{N}}=O\left(\delta^{-\frac{N-2}{2} q}|\log \delta|\right),
$$

as $\delta \rightarrow 0^{+}$, uniformly in $a \in K$ and $|\tau|<\eta^{-1}$.

Proof. We only prove the first inequality. Arguing as in the previous lemma, we have

$$
\begin{aligned}
\int_{\Omega \backslash B_{r \delta^{2}}(a)} U_{\delta, \xi}^{q} \frac{\left|x_{h}-\xi_{h}\right|^{\nu_{1}}}{|x-a|^{\nu_{2}}} & =C \int_{\frac{\Omega \backslash B_{r \delta 2}(a)-a}{\delta}} \delta^{N+\nu_{1}-\frac{N-2}{2} q-\nu_{2}} \frac{|y-\tau|^{\nu_{1}}}{\left(1+|y|^{2}\right)^{q \frac{N-2}{2}}|y|^{\nu_{2}}} d y \\
& \leq C \delta^{N+\nu_{1}-\frac{N-2}{2} q-\nu_{2}} \int_{B_{R / \delta} \backslash B_{r \delta}} \frac{|y-\tau|^{\nu_{1}}}{\left(1+|y|^{2}\right)^{q \frac{N-2}{2}}|y|^{\nu_{2}}} d y,
\end{aligned}
$$

whence the thesis follows.

Lemma A.8. Let $R, q_{1}, q_{2}>0$. Then there exists $C>0$ such that

$$
\int_{\Omega} U_{\delta_{1}, \xi_{1}}^{q_{1}} U_{\delta_{2}, \xi_{2}}^{q_{2}} \leq C \delta_{1}^{\frac{N-2}{2} q_{1}} \delta_{2}^{\frac{N-2}{2} q_{2}}+C \delta_{1}^{\frac{N-2}{2} q_{1}} \int_{\Omega} U_{\delta_{2}, \xi_{2}}^{q_{2}}+C \delta_{2}^{\frac{N-2}{2} q_{2}} \int_{\Omega} U_{\delta_{1}, \xi_{1}}^{q_{1}}
$$

for every $\xi_{1}, \xi_{2} \in \Omega$ such that $\operatorname{dist}\left(\xi_{i}, \partial \Omega\right)>2 R$ and $\left|\xi_{1}-\xi_{2}\right|>2 R$.

Proof. We can split the integral over $\Omega$ using the fact that

$$
\Omega=\left(\Omega \backslash\left(B_{R}\left(\xi_{1}\right) \cup B_{R}\left(\xi_{2}\right)\right)\right) \cup B_{R}\left(\xi_{1}\right) \cup B_{R}\left(\xi_{2}\right) .
$$

Then, the thesis follows using the positivity of $U_{\delta, \xi}$, and the fact that $0 \leq U_{\delta, \xi} \leq C \delta^{\frac{N-2}{2}}$ in $\mathbb{R}^{N} \backslash B_{R}(\xi)$.

Similarly:

Lemma A.9. Let $R, q_{1}, q_{2}>0$. Then there exists $C>0$ such that

$$
\int_{\Omega} U_{\delta_{1}, \xi_{1}}^{q_{1}} U_{\delta_{2}, \xi_{2}}^{q_{2}} \frac{\left|x-\xi_{1}\right|^{\nu_{1}}}{|x-a|^{\nu_{2}}} \leq C \delta_{1}^{\frac{N-2}{2} q_{1}} \delta_{2}^{\frac{N-2}{2} q_{2}}+C \delta_{1}^{\frac{N-2}{2} q_{1}} \int_{\Omega} U_{\delta_{2}, \xi_{2}}^{q_{2}}+C \delta_{2}^{\frac{N-2}{2} q_{2}} \int_{\Omega} U_{\delta_{1}, \xi_{1}}^{q_{1}} \frac{\left|x-\xi_{1}\right|^{\nu_{1}}}{|x-a|^{\nu_{2}}}
$$

for every $\xi_{1}, \xi_{2}, a \in \Omega$ such that $\operatorname{dist}\left(\xi_{i}, \partial \Omega\right)>2 R,\left|\xi_{1}-\xi_{2}\right|>2 R,\left|\xi_{1}-a\right|<R / 2, \nu_{1} \geq 0$ and $0 \leq \nu_{2}<N$. 


\section{Appendix B.}

In this appendix we provide the details for the estimates (3.10).

Estimate of $(I)$ : integrating by parts we deduce that

$$
\begin{aligned}
\delta_{i, n}^{2} \int_{\Omega_{n}} \nabla\left(P_{n} \psi_{i, n}^{l}\right) \cdot \nabla\left(P_{n} \psi_{i, n}^{k}\right) & =\delta_{i, n}^{2} \int_{\Omega_{n}} p U_{i, n}^{p-1} \psi_{i, n}^{k}\left(P_{n} \psi_{i, n}^{l}\right) \\
= & \delta_{i, n}^{2} \int_{\Omega_{n}} p U_{i, n}^{p-1} \psi_{i, n}^{k} \psi_{i, n}^{l}+\delta_{i, n}^{2} \int_{\Omega_{n}} p U_{i, n}^{p-1} \psi_{i, n}^{k}\left(P_{n} \psi_{i, n}^{l}-U_{i, n}^{l}\right) .
\end{aligned}
$$

Due to Corollary A.2, it is not difficult to show that the last term tends to 0 as $n \rightarrow \infty$. We consider here in full details the case $l, k=1, \ldots, N$ (the others can be treated similarly): first, we observe that Corollary A.2 is applicable since $\left\{\xi_{i, n}\right\} \subset K$ for some compact set $K \Subset \Omega$, at least for $\eta>0$ small enough. Then, using also Lemmas A.3 and A.7, and recalling that $\varepsilon_{n} \simeq \delta_{i, n}^{2}$ since $\eta$ is fixed, we deduce that

$$
\begin{gathered}
\delta_{i, n}^{2}\left|\int_{\Omega_{n}} U_{i, n}^{p-1} \psi_{i, n}^{k}\left(P_{n} \psi_{i, n}^{l}-U_{i, n}^{l}\right)\right| \leq C \delta_{i, n} \int_{\Omega_{n}} U_{i, n}^{\frac{4+N}{N-2}}\left|x_{k}-\xi_{k}^{i, n}\right|\left(\delta_{i, n}^{\frac{N-2}{2}}+\frac{\delta_{i, n}^{\frac{3}{2} N-4}}{\left|x-a_{i}\right|^{N-2}}\right) \\
=C \delta_{i, n}^{\frac{N}{2}} \int_{\Omega_{n}} U_{i, n}^{\frac{4+N}{N-2}}\left|x_{k}-\xi_{k}^{i, n}\right|+C \delta_{i, n}^{\frac{3}{2} N-3} \int_{\Omega_{n}} U_{i, n}^{\frac{4+N}{N-2}} \frac{\left|x_{k}-\xi_{k}^{i, n}\right|}{\left|x-a_{i}\right|^{N-2}} \\
\leq C \delta_{i, n}^{\frac{N}{2}+N+1-\frac{4+N}{2}}+C \delta_{i, n}^{\frac{3}{2} N-3+N+1-\frac{4+N}{2}-N+2}=o(1)
\end{gathered}
$$

as $n \rightarrow \infty$.

Coming back to (B.1), using the explicit expression of $U_{i, n}$ and $\psi_{i, n}^{k}$, it is not difficult to check that

$$
\delta_{i, n}^{2} \int_{\Omega_{n}} \nabla\left(P_{n} \psi_{i, n}^{l}\right) \cdot \nabla\left(P_{n} \psi_{i, n}^{k}\right)=\sigma_{l k}+o(1)
$$

where $o(1) \rightarrow 0$ as $n \rightarrow \infty$, and the values $\sigma_{l k}$ are defined in (3.11). Therefore, as $n \rightarrow \infty$

$$
\begin{aligned}
\delta_{i, n}^{2}\left\|w_{i, n}\right\|_{H_{0}^{1}\left(\Omega_{n}\right)}^{2} & =\sum_{l, k=0}^{N} c_{i, n}^{l} c_{i, n}^{k} \delta_{i, n}^{2} \int_{\Omega_{n}} \nabla\left(P_{n} \psi_{i, n}^{l}\right) \cdot \nabla\left(P_{n} \psi_{i, n}^{k}\right) \\
& =\sum_{l, k=0}^{N} c_{i, n}^{l} c_{i, n}^{k}\left(\sigma_{l k}+o(1)\right)=\sum_{l=0}^{N}\left(c_{i, n}^{l}\right)^{2} \sigma_{l l}+o(1) \sum_{l, k=0}^{N} c_{i, n}^{l} c_{i, n}^{k} .
\end{aligned}
$$

Estimate of $(I I)$ : we aim at proving the second equality in (3.10). Since $\phi_{i, n} \in K_{i, n}^{\perp}$, we know that $\left\langle\phi_{i, n}, P_{n} \psi_{i, n}^{l}\right\rangle_{H_{0}^{1}}=0$, i.e.

$$
0=\int_{\Omega_{n}} \nabla\left(P_{n} \psi_{i, n}^{l}\right) \cdot \nabla \phi_{i, n}=p \int_{\Omega_{n}} U_{i, n}^{p-1} \phi_{i, n} \psi_{i, n}^{l} .
$$

As a consequence

$$
|(I I)| \leq \delta_{i, n}^{2}[\underbrace{\int_{\Omega_{n}}\left|\left(P_{n} U_{i, n}\right)^{\frac{4}{N-2}}-U_{i, n}^{\frac{4}{N-2}}\right|\left|\phi_{i, n}\right|\left|w_{i, n}\right|}_{=:(I I .1)}+\sum_{l=0}^{N} c_{i, n}^{l} \underbrace{\int_{\Omega_{n}} U_{i, n}^{\frac{4}{N-2}}\left|\phi_{i, n}\right|\left|P_{n} \psi_{i, n}^{l}-\psi_{i, n}^{l}\right|}_{=:(I I .2)}] .
$$


Concerning the first integral, by the Hölder and the Sobolev inequalities

$$
\begin{aligned}
|(I I .1)| & \leq\left|\left(P_{n} U_{i, n}\right)^{\frac{4}{N-2}}-U_{i, n}^{\frac{4}{N-2}}\right|_{\frac{N}{2}}\left|\phi_{i, n}\right|_{2^{*}}\left|w_{i, n}\right|_{2^{*}} \\
& \leq C\left|\left(P_{n} U_{i, n}\right)^{\frac{4}{N-2}}-U_{i, n}^{\frac{4}{N-2}}\right|_{\frac{N}{2}}\left\|\phi_{i, n}\right\|\left\|w_{i, n}\right\| .
\end{aligned}
$$

We recall that $\left\|\phi_{i, n}\right\|=1$ for every $n$. Moreover, since $0 \leq P_{n} U_{i, n} \leq U_{i, n}$ in $\Omega_{n}$ by the maximum principle, applying Lemma A.4 we have

$$
\begin{aligned}
\left|\left(P_{n} U_{i, n}\right)^{\frac{4}{N-2}}-U_{i, n}^{\frac{4}{N-2}}\right|_{\frac{N}{2}} & \leq\left|U_{i, n}^{\frac{4}{N-2}-1}\left(P_{n} U_{i, n}-U_{i, n}\right)\right|_{\frac{N}{2}}+\left|\left(P_{n} U_{i, n}-U_{i, n}\right)^{\frac{4}{N-2}}\right|_{\frac{N}{2}} \\
& \leq 2\left|U_{i, n}^{\frac{4}{N-2}-1}\left(P_{n} U_{i, n}-U_{i, n}\right)\right|_{\frac{N}{2}}=2\left(\int_{\Omega_{n}} U_{i, n}^{\frac{(6-N) N}{2(N-2)}}\left|P_{n} U_{i, n}-U_{i, n}\right|^{\frac{N}{2}}\right)^{\frac{2}{N}}
\end{aligned}
$$

Thus, using Corollary A.2, the fact that $\varepsilon_{n} \simeq \delta_{i, n}^{2}$ (see ansatz (2.5)), and Lemmas A.6 and A.7, we obtain for $N=3$

$$
\begin{aligned}
& \mid\left(P_{n} U_{i, n}\right)^{\frac{4}{N-2}}-\left.U_{i, n}^{\frac{4}{N-2}}\right|_{\frac{N}{2}} \\
& \leq C \delta_{i, n}^{\frac{N-2}{2}}\left(\int_{\Omega_{n}} U_{i, n}^{\frac{(6-N) N}{2(N-2)}}\right)^{\frac{2}{N}}+C \delta_{i, n}^{\frac{3}{2} N-3}\left(\int_{\Omega_{n}} \frac{U_{i, n}^{\frac{(6-N) N}{2(N-2)}}}{\left|x-a_{i}\right|^{\frac{N-2}{2} N}}\right)^{\frac{2}{N}}=C \delta_{i, n}^{N-2},
\end{aligned}
$$

and similarly for $N=4$

$$
\left|\left(P_{n} U_{i, n}\right)^{\frac{4}{N-2}}-U_{i, n}^{\frac{4}{N-2}}\right|_{\frac{N}{2}} \leq C \delta_{i, n}^{N-2}\left|\log \delta_{i, n}\right|^{\frac{2}{N}} .
$$

Thus, coming back to (B.4), we proved that $|(I I .1)|=o(1)\left\|w_{i, n}\right\|_{H_{0}^{1}\left(\Omega_{n}\right)}$.

Let us consider now $|(I I .2)|$. By the Hölder and the Sobolev inequalities

$$
|(I I .2)| \leq C\left\|\phi_{i, n}\right\|\left|U_{i, n}^{\frac{4}{N-2}}\left(P_{n} \psi_{i, n}^{l}-\psi_{i, n}^{l}\right)\right|_{\frac{2 N}{N+2}} .
$$

The right hand side can be controlled using Corollary A.2 and Lemmas A.6 and A.7. We focus on the case $l=1, \ldots, N$, which is completely analogue with respect to $l=0$, and we compute

$$
\begin{aligned}
|(I I .2)| & \leq\left(\int_{\Omega_{n}} U_{i, n}^{\frac{8 N}{(N-2)(N+2)}}\left|P_{n} \psi_{i, n}^{l}-\psi_{i, n}^{l}\right|^{\frac{2 N}{N+2}}\right)^{\frac{N+2}{2 N}} \\
& \leq C\left(\int_{\Omega_{n}} U_{i, n}^{\frac{8 N}{(N-2)(N+2)}}\left(\delta_{i, n}^{\frac{N-2}{2}}+\frac{\delta_{i, n}^{\frac{3}{2} N-4}}{\left|x-a_{i}\right|^{N-2}}\right)^{\frac{2 N}{N+2}}\right)^{\frac{N+2}{2 N}} \\
& \leq C \delta_{i, n}^{\frac{N-2}{2}}\left(\int_{\Omega_{n}} U_{i, n}^{\frac{8 N}{(N-2)(N+2)}}\right)^{\frac{N+2}{2 N}}+C \delta_{i, n}^{\frac{3}{2} N-4}\left(\int_{\Omega_{n}} \frac{U_{i, n}^{\frac{8 N}{(N-2)(N+2)}}}{\left|x-a_{i}\right|^{\frac{2 N(N-2)}{N+2}}}\right)^{\frac{N+2}{2 N}} \leq C \delta_{i, n}^{N-3} .
\end{aligned}
$$

Coming back to (B.3) (and recalling that $N=3,4$ ), we proved the validity of the second estimate in (3.10), as desired.

Estimate of $(I I I)$ : we show that $(I I I)=o\left(\delta_{i, n}^{2}\right)$ as $n \rightarrow \infty$, focusing here on the case $l=0$; the one $l=1, \ldots, N$ is, once again, completely analogue. Recalling that $0 \leq P_{n} U_{i, n} \leq U_{i, n}$, using the 
Hölder and the Sobolev inequalities we compute

$$
\begin{aligned}
\delta_{i, n}^{2} \mid \int_{\Omega_{n}} & \left(P_{n} U_{i, n}\right)^{\frac{p-3}{2}}\left(P_{n} U_{j, n}\right)^{\frac{p+1}{2}} \phi_{i, n}\left(P_{n} \psi_{i, n}^{l}\right) \mid \\
& =\delta_{i, n}^{2}\left|\int_{\Omega_{n}}\left(P_{n} U_{i, n}\right)^{\frac{p-3}{2}}\left(P_{n} U_{j, n}\right)^{\frac{p+1}{2}} \phi_{i, n}\left[\psi_{i, n}^{l}+\left(P_{n} \psi_{i, n}^{l}-\psi_{i, n}^{l}\right)\right]\right| \\
& \leq \delta_{i, n}^{2} \int_{\Omega_{n}} U_{i, n}^{\frac{p-3}{2}} U_{j, n}^{\frac{p+1}{2}}\left|\phi_{i, n}\right|\left|\psi_{i, n}^{l}\right|+\delta_{i, n}^{2} \int_{\Omega_{n}} U_{i, n}^{\frac{p-3}{2}} U_{j, n}^{\frac{p+1}{2}}\left|\phi_{i, n} \| P_{n} \psi_{i, n}^{l}-\psi_{i, n}^{l}\right| \\
& \leq C \delta_{i, n}^{2} \underbrace{\left\|\phi_{i, n}\right\|\left|U_{i, n}^{\frac{p-3}{2}} U_{j, n}^{\frac{p+1}{2}}\right| \psi_{i, n}^{l}||_{\frac{2 N}{N+2}}}_{=:(I I I .1)}+C \delta_{i, n}^{2} \underbrace{\left\|\phi_{i, n}\right\|\left|U_{i, n}^{\frac{p-3}{2}} U_{j, n}^{\frac{p+1}{2}}\right| P_{n} \psi_{i, n}^{l}-\psi_{i, n}^{l}||_{\frac{2 N}{N+2}}}_{=:(I I I .2)}
\end{aligned}
$$

In order to estimate (III.2), we use Corollary A.2, the ansatz (2.5), and the fact that $\left\|\phi_{i, n}\right\|=1$ :

$$
\begin{aligned}
(I I I .2) & \leq C\left|U_{i, n}^{\frac{p-3}{2}} U_{j, n}^{\frac{p+1}{2}}\left(\delta_{i, n}^{\frac{N-4}{2}}+\frac{\delta_{i, n}^{\frac{3}{2} N-4}}{\left|x-a_{i}\right|^{N-2}}\right)\right|_{\frac{2 N}{N+2}} \\
& \leq C \delta_{i, n}^{\frac{N-4}{2}}\left(\int_{\Omega_{n}} U_{i, n}^{\frac{2 N(4-N)}{(N-2)(N+2)}} U_{j, n}^{\frac{2 N^{2}}{(N-2)(N+2)}}\right)^{\frac{N+2}{2 N}} \\
& +C \delta_{i, n}^{\frac{3}{2} N-4}\left(\int_{\Omega_{n}} \frac{U_{i, n}^{\frac{2 N(4)-N)}{(N-2)(N+2)}} U_{j, n}^{\frac{2 N^{2}}{(N-2)(N+2)}}}{\left|x-a_{i}\right|^{\frac{2 N(N-2)}{(N+2)}}}\right)^{\frac{N+2}{2 N}}
\end{aligned}
$$

By Lemmas A.6 and A.8,

$$
\begin{aligned}
& \delta_{i, n}^{\frac{N-4}{2}}\left(\int_{\Omega_{n}} U_{i, n}^{\frac{2 N(4-N)}{(N-2)(N+2)}} U_{j, n}^{\frac{2 N^{2}}{(N-2)(N+2)}}\right)^{\frac{N+2}{2 N}} \\
& \quad \leq C \delta_{i, n}^{\frac{N-4}{2}}\left(\delta_{i, n}^{\frac{N(4-N)}{N+2}} \delta_{j, n}^{\frac{N^{2}}{N+2}}+\delta_{i, n}^{\frac{N(4-N)}{N+2}} \delta_{j, n}^{\frac{2 N}{N+2}}+C \delta_{j, n}^{\frac{N^{2}}{N+2}} \int_{\Omega_{n}} U_{i, n}^{\frac{2 N(4-N)}{(N-2)(N+2)}}\right)^{\frac{N+2}{2 N}}
\end{aligned}
$$

and discussing separately the case $N=3$ and $N=4$, it is not difficult to check that in both cases

$$
\delta_{i, n}^{\frac{N-4}{2}}\left(\int_{\Omega_{n}} U_{i, n}^{\frac{2 N(4-N)}{(N-2)(N+2)}} U_{j, n}^{\frac{2 N^{2}}{(N-2)(N+2)}}\right)^{\frac{N+2}{2 N}} \leq C \delta_{j, n}=o(1)
$$

as $n \rightarrow \infty$. The second term on the right hand side in (B.6) can be controlled using Lemmas A.6 and A.9, in a similar way:

$$
\begin{aligned}
& \delta_{i, n}^{\frac{3}{2} N-4}\left(\int_{\Omega_{n}} \frac{U_{i, n}^{\frac{2 N(4-N)}{(N-2)(N+2)}} U_{j, n}^{\frac{2 N^{2}}{(N-2)(N+2)}}}{\left|x-a_{i}\right|^{\frac{2 N(N-2)}{(N+2)}}}\right)^{\frac{N+2}{2 N}} \\
& \quad \leq C \delta_{i, n}^{\frac{3}{2} N-4}\left(\delta_{i, n}^{\frac{N(4-N)}{N+2}} \delta_{j, n}^{\frac{N^{2}}{N+2}}+\delta_{i, n}^{\frac{N(4-N)}{N+2}} \delta_{j, n}^{\frac{2 N}{N+2}}+C \delta_{j, n}^{\frac{N^{2}}{N+2}} \delta_{i, n}^{N-\frac{N(4-N)}{N+2}-\frac{2 N(N-2)}{N+2}}\right)^{\frac{N+2}{2 N}}=o(1)
\end{aligned}
$$


as $n \rightarrow \infty$. Therefore, (III.2) $\rightarrow 0$ as $n \rightarrow \infty$. Concerning the term (III.1), by Lemmas A.3, A.6 and A.8, we obtain

$$
\begin{aligned}
|(I I I .1)| & \leq C \delta_{i, n}^{-1}\left|U_{i, n}^{\frac{p-1}{2}} U_{j, n}^{\frac{p+1}{2}}\right|_{\frac{2 N}{N+2}} \\
& \leq C \delta_{i, n}^{-1}\left(\int_{\Omega_{n}} U_{i, n}^{\frac{4 N}{(N-2)(N+2)}} U_{j, n}^{\frac{2 N^{2}}{(N-2)(N+2)}}\right)^{\frac{N+2}{2 N}} \\
& C \delta_{i, n}^{-1}\left(\delta_{i, n}^{\frac{2 N}{N+2}} \delta_{j, n}^{\frac{N^{2}}{N+2}}+\delta_{i, n}^{\frac{2 N}{N+2}} \delta_{j, n}^{\frac{2 N}{N+2}}+\delta_{i, n}^{\frac{2 N}{N+2}} \delta_{j, n}^{\frac{N^{2}}{N+2}}\right)^{\frac{N+2}{2 N}} \leq C \delta_{j, n}=o(1)
\end{aligned}
$$

as $n \rightarrow \infty$.

To sum up we showed that (III.1) and (III.2) tend to 0 as $n \rightarrow \infty$, and coming back to (B.5) we infer that $(I I I)=o\left(\delta_{i, n}^{2}\right)$, as desired.

Estimate of $(I V)$ : this is similar to the estimate of $(I I I)$, and this time we focus on $l=1, \ldots, N$. We have

$$
\begin{aligned}
& \delta_{i, n}^{2}\left|\int_{\Omega_{n}}\left(P_{n} U_{i, n}\right)^{\frac{p-1}{2}}\left(P_{n} U_{j, n}\right)^{\frac{p-1}{2}} \phi_{i, n}\left(P_{n} \psi_{i, n}^{l}\right)\right| \\
& \leq C \delta_{i, n}^{2} \underbrace{\left\|\phi_{i, n}\right\|\left|U_{i, n}^{\frac{p-1}{2}} U_{j, n}^{\frac{p-1}{2}}\right| \psi_{i, n}^{l}||_{\frac{2 N}{N+2}}}_{=:(I V .1)}+C \delta_{i, n}^{2} \underbrace{\left\|\phi_{i, n}\right\|\left|U_{i, n}^{\frac{p-1}{2}} U_{j, n}^{\frac{p-1}{2}}\right| P_{n} \psi_{i, n}^{l}-\psi_{i, n}^{l}||_{\frac{2 N}{N+2}}}_{=:(I V .2)}
\end{aligned}
$$

The term (IV.2) can be controlled using Corollary A.2:

$$
\begin{aligned}
(I V .2) & \leq C\left|U_{i, n}^{\frac{p-1}{2}} U_{j, n}^{\frac{p-1}{2}}\left(\delta_{i, n}^{\frac{N-2}{2}}+\frac{\delta_{i, n}^{\frac{3}{2} N-4}}{\left|x-a_{i}\right|^{N-2}}\right)\right|_{\frac{2 N}{N+2}} \\
& \leq C \delta_{i, n}^{\frac{N-2}{2}}\left(\int_{\Omega_{n}} U_{i, n}^{\frac{4 N}{(N-2)(N+2)}} U_{j, n}^{\frac{4 N}{(N-2)(N+2)}}\right)^{\frac{N+2}{2 N}} \\
& +C \delta_{i, n}^{\frac{3}{2} N-4}\left(\int_{\Omega_{n}} \frac{U_{i, n}^{\frac{4 N}{(N-2)(N+2)}} U_{j, n}^{\frac{4 N}{(N-2)(N+2)}}}{\left|x-a_{i}\right|^{\frac{2 N(N-2)}{(N+2)}}}\right)^{\frac{N+2}{2 N}} .
\end{aligned}
$$

By Lemmas A.6 and A.8, the first integral on the right hand side gives

$$
\delta_{i, n}^{\frac{N-2}{2}}\left(\int_{\Omega_{n}} U_{i, n}^{\frac{4 N}{(N-2)(N+2)}} U_{j, n}^{\frac{4 N}{(N-2)(N+2)}}\right)^{\frac{N+2}{2 N}} \leq C \delta_{i, n}^{\frac{N-2}{2}}\left(\delta_{i, n}^{\frac{2 N}{N+2}} \delta_{j, n}^{\frac{2 N}{N+2}}\right)^{\frac{N+2}{2 N}}=o(1)
$$

and, by Lemmas A.6 and A.9, the second integral gives

$$
\begin{aligned}
& \delta_{i, n}^{\frac{3}{2} N-4}\left(\int_{\Omega_{n}} \frac{U_{i, n}^{\frac{4 N}{(N-2)(N+2)}} U_{j, n}^{\frac{4 N}{(N-2)(N+2)}}}{\left|x-a_{i}\right|^{\frac{2 N(N-2)}{(N+2)}}}\right)^{\frac{N+2}{2 N}} \\
& \quad \leq C \delta_{i, n}^{\frac{3}{2} N-4}\left(\delta_{i, n}^{\frac{2 N}{N+2}} \delta_{j, n}^{\frac{2 N}{N+2}}+\delta_{i, n}^{\frac{N(4-N)}{N+2}} \delta_{j, n}^{\frac{2 N}{N+2}}\right)^{\frac{N+2}{2 N}}=o(1)
\end{aligned}
$$


as $n \rightarrow \infty$. As far as (IV.1) is concerned, using Lemmas A.3, A.7 and A.9, we infer that

$$
\begin{aligned}
|(I V .1)| & \leq C \delta_{i, n}^{-1}\left|U_{i, n}^{\frac{p-1}{2}+\frac{N}{N-2}} U_{j, n}^{\frac{p-1}{2}}\right| x_{l}-\xi_{i, l}^{n}||_{\frac{2 N}{N+2}} \\
& \leq C \delta_{i, n}^{-1}\left(\int_{\Omega_{n}} U_{i, n}^{\frac{2 N}{N-2}} U_{j, n}^{\frac{4 N}{(N-2)(N+2)}}\left|x_{l}-\xi_{i, l}^{n}\right|^{\frac{2 N}{N+2}}\right)^{\frac{N+2}{2 N}} \\
& \leq C \delta_{i, n}^{-1}\left(\delta_{i, n}^{N} \delta_{j, n}^{\frac{2 N}{N+2}}+\delta_{j, n}^{\frac{2 N}{N+2}} \delta_{i, n}^{N-N+\frac{2 N}{N+2}}\right)^{\frac{N+2}{2 N}}=o(1)
\end{aligned}
$$

as $n \rightarrow \infty$. Altogether, we proved that $(I V .1)$ and (IV.2) tend to 0 as $n \rightarrow \infty$, so that $(I V)=o\left(\delta_{i, n}^{2}\right)$ as $n \rightarrow \infty$.

This completes the proof of the validity of the estimates (3.10).

\section{Appendix C.}

In this appendix we show that (3.13) gives (3.14). At first, we notice that

$$
\begin{array}{r}
p \delta_{\kappa, n}^{2} \int_{\tilde{\Omega}_{\kappa, n}}\left(\widehat{P_{n} U_{\kappa, n}}\right)^{p-1} \tilde{\phi}_{\kappa, n} \psi=p \delta_{\kappa, n}^{2} \int_{\tilde{\Omega}_{\kappa, n}} U_{\kappa, n}^{p-1}\left(\xi_{\kappa, n}+\delta_{\kappa, n} \cdot\right) \tilde{\phi}_{\kappa, n} \psi \\
+p \delta_{\kappa, n}^{2} \int_{\tilde{\Omega}_{\kappa, n}}\left[\left(\widehat{P_{n} U_{\kappa, n}}\right)^{p-1}-U_{\kappa, n}^{p-1}\left(\xi_{\kappa, n}+\delta_{\kappa, n} \cdot\right)\right] \tilde{\phi}_{\kappa, n} \psi
\end{array}
$$

Arguing as in Appendix B, estimate of term (II.1), it can be proved that the last integral on the right hand side tends to 0 as $n \rightarrow \infty$. Moreover, the first integral can be explicitly computed:

$$
U_{\kappa, n}^{p-1}\left(\xi_{\kappa, n}+\delta_{\kappa, n} y\right) \psi(y)=\alpha_{N}^{p-1}\left(\frac{1}{1+|y|^{2}}\right)^{2} \psi(y)=U_{0,1}^{p-1}(y) \psi(y) \in L^{\frac{2 N}{N+2}}\left(\mathbb{R}^{N}\right)
$$

(where we recall that $U_{1,0}$ denotes the standard bubble with $\delta=1$ and $\xi=0$ ), and hence by weak convergence $\tilde{\phi}_{\kappa, n} \rightarrow \tilde{\phi}_{\kappa}$ in $L^{2^{*}}\left(\mathbb{R}^{N}\right)$

$$
\begin{aligned}
p \delta_{\kappa, n}^{2} \int_{\tilde{\Omega}_{\kappa, n}}\left(\widehat{P_{n} U_{\kappa, n}}\right)^{p-1} \tilde{\phi}_{\kappa, n} \psi & =p \delta_{\kappa, n}^{2} \int_{\tilde{\Omega}_{\kappa, n}} U_{\kappa, n}^{p-1}\left(\xi_{\kappa, n}+\delta_{\kappa, n} \cdot\right) \tilde{\phi}_{\kappa, n} \psi+o(1) \\
& =p \int_{\tilde{\Omega}_{\kappa, n}} U_{1,0}^{p-1} \tilde{\phi}_{\kappa, n} \psi+o(1)=p \int_{\mathbb{R}^{N}} U_{1,0}^{p-1} \tilde{\phi}_{i} \psi+o(1)
\end{aligned}
$$

as $n \rightarrow \infty$.

Now we show that the remaining terms on the right hand side of (3.13) tend to 0 as $n \rightarrow \infty$. We start observing that, if $j \neq \kappa$, for any compact set $K \subset \mathbb{R}^{N}$ there exists $C>0$ (depending on $K$ ) such that for sufficiently large $n$

$$
\begin{aligned}
\inf _{y \in K}\left|\xi_{\kappa, n}+\delta_{\kappa, n} y-\xi_{j, n}\right| & \geq\left|\xi_{\kappa, n}-\xi_{j, n}\right|-C \delta_{\kappa, n} \\
& \geq \frac{1}{2}\left|a_{\kappa}-a_{j}\right|-C \delta_{\kappa, n} \geq \frac{1}{4}\left|a_{\kappa}-a_{j}\right| .
\end{aligned}
$$


Thus, for any $j \neq \kappa$

$$
\begin{aligned}
& \delta_{\kappa, n}^{2}\left|\int_{\tilde{\Omega}_{\kappa, n}}\left(\widehat{P_{n} U_{\kappa, n}}\right)^{\frac{p-3}{2}}\left(\widehat{P_{n} U_{j, n}}\right)^{\frac{p+1}{2}} \tilde{\phi}_{\kappa, n} \psi\right| \\
& \leq C \delta_{\kappa, n}^{2} \int_{\tilde{\Omega}_{\kappa, n}} U_{\kappa, n}^{\frac{p-3}{2}}\left(\xi_{\kappa, n}+\delta_{\kappa, n} \cdot\right) U_{j, n}^{\frac{p+1}{2}}\left(\xi_{\kappa, n}+\delta_{\kappa, n} \cdot\right)\left|\tilde{\phi}_{\kappa, n} \| \psi\right| \\
& \leq C \delta_{\kappa, n}^{2} \int_{\operatorname{supp} \psi}\left(\frac{\delta_{j, n}}{\delta_{j, n}^{2}+\left|\xi_{\kappa, n}+\delta_{\kappa, n} y-\xi_{j, n}\right|^{2}}\right)^{\frac{N}{2}}\left(\frac{\delta_{\kappa, n}^{-1}}{1+|y|^{2}}\right)^{\frac{4-N}{2}}\left|\tilde{\phi}_{\kappa, n}(y)\right||\psi(y)| d y \\
& \leq C \delta_{\kappa, n}^{\frac{N}{2}} \delta_{j, n}^{\frac{N}{2}} \int_{\operatorname{supp} \psi}\left|\tilde{\phi}_{\kappa, n}(y)\right||\psi(y)| d y \leq C \delta_{\kappa, n}^{\frac{N}{2}} \delta_{j, n}^{\frac{N}{2}}\left|\tilde{\phi}_{\kappa, n}\right|_{2^{*}}|\psi|_{\frac{2 N}{N+2}}=o(1)
\end{aligned}
$$

as $n \rightarrow \infty$.

Similarly, always for any $j \neq i$

$$
\begin{aligned}
\delta_{\kappa, n}^{2} \mid & \int_{\tilde{\Omega}_{\kappa, n}}\left(\widehat{P_{n} U_{\kappa, n}}\right)^{\frac{p-1}{2}}\left(\widehat{P_{n} U_{j, n}}\right)^{\frac{p-1}{2}} \tilde{\phi}_{j, n} \psi \mid \\
& \leq C \delta_{\kappa, n}^{2} \int_{\tilde{\Omega}_{\kappa, n}} \frac{\delta_{j, n}}{\delta_{j, n}^{2}+\left|\xi_{\kappa, n}+\delta_{\kappa, n} y-\xi_{j, n}\right|^{2}} \frac{\delta_{\kappa, n}^{-1}}{1+|y|^{2}}\left|\tilde{\phi}_{j, n}(y)\right||\psi(y)| d y \\
& \leq C \delta_{\kappa, n} \delta_{j, n} \int_{\operatorname{supp} \psi}\left|\tilde{\phi}_{j, n}(y)\right||\psi(y)| d y=o(1)
\end{aligned}
$$

as $n \rightarrow \infty$.

Finally, also the last term on the right hand side in (3.13) tends to 0 , since $\left\|\tilde{h}_{\kappa, n}\right\|_{H_{0}^{1}\left(\tilde{\Omega}_{\kappa, n}\right)}=$ $\left\|h_{\kappa, n}\right\|_{H_{0}^{1}\left(\Omega_{n}\right)},\left\|\tilde{w}_{\kappa, n}\right\|_{H_{0}^{1}\left(\tilde{\Omega}_{\kappa, n}\right)}=\left\|w_{\kappa, n}\right\|_{H_{0}^{1}\left(\Omega_{n}\right)}$, both $h_{\kappa, n}$ and $w_{\kappa, n}$ converge to 0 strongly in $H_{0}^{1}\left(\Omega_{n}\right)$ $\left(h_{\kappa, n}\right.$ by assumption, $w_{\kappa, n}$ by step 1 ), and $\tilde{\phi}_{\kappa, n} \rightarrow \tilde{\phi}_{i}$ weakly.

\section{APPENDIX D.}

In this appendix we prove the validity of the estimates (3.23) and (3.26). In order to ease the notation, we write $P$ and $U_{i}$ instead of $P_{\varepsilon}$ and $U_{\delta_{i}, \xi_{i}}$.

We start with the proof of (3.23).

Estimate of $\left|\tilde{R}_{\mathbf{d}, \boldsymbol{\tau}, \varepsilon}^{i}\right|_{L^{\frac{2 N}{N+2}}\left(\Omega_{\varepsilon}\right)}$. By Lemma A.4 and the fact that $0 \leq P U_{i} \leq U_{i}$,

$$
\left|P U_{i}^{p}-U_{i}^{p}\right| \leq C\left(\left|U_{i}\right|^{p-1}\left|P U_{i}-U_{i}\right|+\left|P U_{i}-U_{i}\right|^{p}\right) \leq C\left|U_{i}\right|^{p-1}\left|P U_{i}-U_{i}\right|
$$

Therefore, recalling that $\varepsilon \simeq \delta_{i}^{2}$ by (2.5) (we fixed $\eta$ from the beginning), Corollary A.2 and Lemmas A.6 and A.7 give

$$
\begin{aligned}
\left(\int_{\Omega_{\varepsilon}} \mid P U_{i}^{p}\right. & \left.-\left.U_{i}^{p}\right|^{\frac{2 N}{N+2}}\right)^{\frac{N+2}{2 N}} \leq C\left(\int_{\Omega_{\varepsilon}} U_{i}^{\frac{8 N}{(N-2)(N+2)}}\left|P U_{i}-U_{i}\right|^{\frac{2 N}{N+2}}\right)^{\frac{N+2}{2 N}} \\
& \leq C\left(\int_{\Omega_{\varepsilon}} U_{i}^{\frac{8 N}{(N-2)(N+2)}}\left(\delta_{i}^{\frac{N-2}{2}}+\frac{\delta_{i}^{\frac{3}{2}(N-2)}}{\left|x-a_{i}\right|^{N-2}}\right)^{\frac{2 N}{N+2}}\right)^{\frac{N+2}{2 N}} \\
& \leq C\left(\delta_{i}^{\frac{2 N(N-2)}{N+2}}+\delta_{i}^{\frac{3 N(N-2)}{N+2}+\frac{N(2-N)}{N+2}}\right)^{\frac{N+2}{2 N}} \leq C \delta_{i}^{N-2} \leq C \varepsilon^{\frac{N-2}{2}} .
\end{aligned}
$$


We also have, by Lemmas A.6 and A.8

$$
\begin{aligned}
\left|\left(P U_{j}\right)^{\frac{p+1}{2}}\left(P U_{i}\right)^{\frac{p-1}{2}}\right|_{\frac{2 N}{N+2}} & \leq\left|U_{j}^{\frac{p+1}{2}} U_{i}^{\frac{p-1}{2}}\right|_{\frac{2 N}{N+2}} \\
& =\left(\int_{\Omega_{\varepsilon}} U_{j}^{\frac{2 N^{2}}{(N+2)(N-2)}} U_{i}^{\frac{4 N}{(N+2)(N-2)}}\right)^{\frac{N+2}{2 N}} \leq C \delta_{i} \delta_{j} \leq C \varepsilon .
\end{aligned}
$$

This estimate and (D.1) imply that, for $\varepsilon>0$ small enough, there exists $C>0$ such that

$$
\left|\tilde{R}_{\mathbf{d}, \boldsymbol{\tau}, \varepsilon}^{i}\right|_{L^{\frac{2 N}{N+2}}\left(\Omega_{\varepsilon}\right)} \leq C\left(\varepsilon^{\frac{N-2}{2}}+\varepsilon\right) \leq C \varepsilon^{\frac{N-2}{2}},
$$

where we used the fact that $N=3$, so that $\varepsilon \leq \varepsilon^{\frac{N-2}{2}}$ for small $\varepsilon$.

Estimate of $\left|\tilde{N}_{\mathbf{d}, \boldsymbol{\tau}, \varepsilon}^{i}(\phi)\right|_{L^{\frac{2 N}{N+2}}\left(\Omega_{\varepsilon}\right)}$. At first, with the aid of Lemma A.4, we compute

$$
\begin{aligned}
\left|\tilde{P}_{\mathbf{d}, \boldsymbol{\tau}, \varepsilon}^{i}(\phi)\right|_{\frac{2 N}{N+2}} & \leq\left.\left. C\left|\left(P U_{i}\right)^{p-2} \phi_{i}^{2}+\right| \phi_{i}\right|^{p}\right|_{\frac{2 N}{N+2}} \leq C\left|U_{i}^{\frac{6-N}{N-2}} \phi_{i}^{2}\right|_{\frac{2 N}{N+2}}+\left|\phi_{i}\right|_{2^{*}}^{\frac{N+2}{N-2}} \\
& \leq C\left|U_{i}\right|_{2^{*}}^{\frac{6-N}{N-2}}\left|\phi_{i}\right|_{2^{*}}^{2}+\left|\phi_{i}\right|_{2^{*}}^{\frac{N+2}{N-2}} \leq C\left\|\phi_{i}\right\|^{2}+\left\|\phi_{i}\right\|^{\frac{N+2}{N-2}}
\end{aligned}
$$

Regarding the remaining term $\tilde{Q}_{\mathbf{d}, \boldsymbol{\tau}, \varepsilon}^{i}(\phi)$, we focus at first on $N=3$. Using Lemma A.5 ${ }^{2}$ and the usual arguments we deduce that

$$
\begin{gathered}
\left|\tilde{Q}_{\mathbf{d}, \boldsymbol{\tau}, \varepsilon}^{i}(\phi)\right|_{\frac{6}{5}} \leq C|| \mu_{j}^{-\frac{1}{p-1}} P U_{j}+\left.\left|\phi_{j}\right|\right|^{3}\left|\phi_{i}\right|^{2} \\
\quad+\left|\mu_{i}^{-\frac{1}{p-1}} P U_{i}+\right| \phi_{i}||\left|\mu_{j}^{-\frac{1}{p-1}} P U_{j}+\right| \phi_{j}||^{2}\left|\phi_{i}\right|\left|\phi_{j}\right| \\
\quad+\left.\left|\mu_{i}^{-\frac{1}{p-1}} P U_{i}+\right| \phi_{i}||^{2}\left|\mu_{j}^{-\frac{1}{p-1}} P U_{j}+\right| \phi_{j}||\left|\phi_{j}\right|^{2}\right|_{\frac{6}{5}} \\
\leq C\left[\left|U_{j}^{3} \phi_{i}^{2}\right|_{\frac{6}{5}}+\left|\phi_{j}^{3} \phi_{i}^{2}\right|_{\frac{6}{5}}+\left|U_{i} U_{j}^{2} \phi_{i} \phi_{j}\right|_{\frac{6}{5}}+\left|U_{i} \phi_{i} \phi_{j}^{3}\right|_{\frac{6}{5}}+\left|U_{j}^{2} \phi_{i}^{2} \phi_{j}\right|_{\frac{6}{5}}\right. \\
\left.\quad+\left|U_{i}^{2} U_{j} \phi_{j}^{2}\right|_{\frac{6}{5}}+\left|U_{i}^{2} \phi_{j}^{3}\right|_{\frac{6}{5}}+\left|U_{j} \phi_{i}^{2} \phi_{j}^{2}\right|_{\frac{6}{5}}\right] \\
\leq C\left[\left|U_{j}\right|_{2^{*}}^{3}\left|\phi_{i}\right|_{2^{*}}^{2}+\left|\phi_{i}\right|_{2^{*}}^{2}\left|\phi_{j}\right|_{2^{*}}^{3}+\left|U_{i}\right|_{2^{*}}\left|U_{j}\right|_{2^{*}}^{2}\left|\phi_{i}\right|_{2^{*}}\left|\phi_{j}\right|_{2^{*}}\right. \\
\quad+\left|U_{i}\right|_{2^{*}}\left|\phi_{i}\right|_{2^{*}}\left|\phi_{j}\right|_{2^{*}}^{3}+\left|U_{j}\right|_{2^{*}}^{2}\left|\phi_{i}\right|_{2^{*}}^{2}\left|\phi_{j}\right|_{2^{*}}+\left|U_{i}\right|_{2^{*}}^{2}\left|U_{j}\right|_{2^{*}}\left|\phi_{j}\right|_{2^{*}}^{2} \\
\left.\quad+\left|U_{i}\right|_{2^{*}}^{2}\left|\phi_{j}\right|_{2^{*}}^{3}+\left|U_{j}\right|_{2^{*}}\left|\phi_{i}\right|_{2^{*}}^{2}\left|\phi_{j}\right|_{2^{*}}^{2}\right] \\
\leq C\left(\|\phi\|^{2}+\|\phi\|^{5}\right)=C\left(\|\phi\|^{2}+\| \phi|| \frac{N+2}{N-2}\right) .
\end{gathered}
$$

Let us consider now the easier case $N=4$. We have

$$
\begin{aligned}
\left|\mu_{j}^{-\frac{1}{2}} P U_{j}+\phi_{j}\right|^{2}\left(\mu_{i}^{-\frac{1}{2}} P U_{i}\right. & \left.+\phi_{i}\right)-\left(\mu_{j}^{-\frac{1}{2}} P U_{j}\right)^{2}\left(\mu_{i}^{-\frac{1}{2}} P U_{i}\right)-\left(\mu_{j}^{-\frac{1}{2}} P U_{\delta_{j}, \xi_{j}}\right)^{2} \phi_{i} \\
& -2\left(\mu_{j}^{-\frac{1}{2}} P U_{\delta_{j}, \xi_{j}}\right)\left(\mu_{i}^{-\frac{1}{2}} P U_{i}\right) \phi_{j}=\mu_{i}^{-\frac{1}{2}} P U_{i} \phi_{j}^{2}+2 \mu_{j}^{-\frac{1}{2}} P U_{j} \phi_{i} \phi_{j}+\phi_{i} \phi_{j}^{2}
\end{aligned}
$$

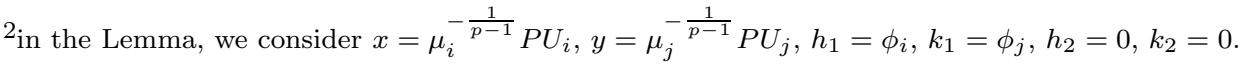


Therefore

$$
\begin{aligned}
\left|\tilde{Q}_{\mathbf{d}, \boldsymbol{\tau}, \varepsilon}^{i}(\phi)\right|_{\frac{4}{3}} & \leq C \sum_{i \neq j}\left(\left|U_{i} \phi_{j}^{2}\right|_{\frac{4}{3}}+\left|U_{j} \phi_{i} \phi_{j}\right|_{\frac{4}{3}}+\left|\phi_{i} \phi_{j}^{2}\right|_{\frac{4}{3}}\right) \\
& \leq C \sum_{i \neq j}\left(\left|U_{i}\right|_{2^{*}}\left|\phi_{j}\right|_{2^{*}}^{2}+\left|U_{j}\right|_{2^{*}}\left|\phi_{i}\right|_{2^{*}}\left|\phi_{j}\right|_{2^{*}}+\left|\phi_{i}\right|_{2^{*}}\left|\phi_{j}\right|_{2^{*}}^{2}\right) \\
& \leq C\left(\|\phi\|^{2}+\|\phi\|^{3}\right)=C\left(\|\phi\|^{2}+\|\phi\|^{\frac{N+2}{N-2}}\right)
\end{aligned}
$$

Collecting (D.3) and (D.4), we conclude that for every $i=1, \ldots, m$, and provided that $\|\phi\|<1$,

$$
\left\|\tilde{N}_{\mathbf{d}, \boldsymbol{\tau}, \varepsilon}^{i}(\phi)\right\|_{\frac{2 N}{N+2}} \leq C\left(\|\phi\|^{2}+\|\phi\|^{\frac{N+2}{N-2}}\right) \leq C\|\phi\|^{2}
$$

for some $C>0$ depending only on the data.

Now we pass to the proof of the validity of (3.26)

We apply again Lemma A.5 (notice that if $N=4$ then $p=3$, while if $N=3$ then $p=5$ ): thus

$$
\begin{aligned}
& \left|\tilde{Q}_{\mathbf{d}, \boldsymbol{\tau}, \varepsilon}^{i}\left(\phi^{1}\right)-\tilde{Q}_{\mathbf{d}, \boldsymbol{\tau}, \varepsilon}^{i}\left(\phi^{2}\right)\right|_{\frac{2 N}{N+2}} \\
& \leq C \frac{p-3}{2} \sum_{j \neq i}|| U_{j}+\left|\phi_{j}^{1}\right|+\left.\left.\left|\phi_{j}^{2}\right|\right|^{\frac{p+1}{2}}|| \phi_{i}^{1}|+| \phi_{i}^{2}||\left|\phi_{i}^{1}-\phi_{i}^{2}\right|\right|_{\frac{2 N}{N+2}} \\
& +C \sum_{j \neq i}|| U_{i}+\left|\phi_{i}^{1}\right|+\left.\left.\left|\phi_{i}^{2}\right|\right|^{\frac{p-3}{2}}\left|U_{j}+\right| \phi_{j}^{1}|+| \phi_{j}^{2}||^{\frac{p-1}{2}}|| \phi_{j}^{1}|+| \phi_{j}^{2}||\left|\phi_{i}^{1}-\phi_{i}^{2}\right|\right|_{\frac{2 N}{N+2}} \\
& +C \sum_{j \neq i}|| U_{i}+\left|\phi_{i}^{1}\right|+\left.\left.\left|\phi_{i}^{2}\right|^{\frac{p-3}{2}}\left|U_{j}+\right| \phi_{j}^{1}|+| \phi_{j}^{2}\right|^{\frac{p-1}{2}}|| \phi_{i}^{1}|+| \phi_{i}^{2}||\left|\phi_{j}^{1}-\phi_{j}^{2}\right|\right|_{\frac{2 N}{N+2}} \\
& +C \sum_{j \neq i}|| U_{i}+\left|\phi_{i}^{1}\right|+\left.\left.\left.\left|\phi_{i}^{2}\right|\right|^{\frac{p-1}{2}}\left|U_{j}+\right| \phi_{j}^{1}|+| \phi_{j}^{2}\right|^{\frac{p-3}{2}}|| \phi_{j}^{1}|+| \phi_{j}^{2}||\left|\phi_{j}^{1}-\phi_{j}^{2}\right|\right|_{\frac{2 N}{N+2}},
\end{aligned}
$$

where we wrote $U_{i}$ for $U_{i}$. To estimate the right hand side, we apply the Hölder and the Sobolev inequalities. We notice at first that the first term survives only if $N=3$. In such case, for any $\phi^{1}, \phi^{2} \in Y_{\varepsilon}$ (recall that then $\left\|\phi^{1}\right\|,\left\|\phi^{2}\right\| \leq C \varepsilon^{\frac{N-2}{2}}$ )

$$
\begin{gathered}
|| U_{j}+\left|\phi_{j}^{1}\right|+\left.\left|\phi_{j}^{2}\right|\right|^{3}|| \phi_{i}^{1}|+| \phi_{i}^{2}\left|\left\|\phi_{i}^{1}-\phi_{i}^{2}||_{\frac{6}{5}} \leq\left|U_{j}+\right| \phi_{j}^{1}|+| \phi_{j}^{2}||_{2^{*}}^{3}\right\| \phi_{i}^{1}\right|+\left|\phi_{i}^{2} \|_{2^{*}}\right| \phi_{i}^{1}-\left.\phi_{i}^{2}\right|_{2^{*}} \\
\leq C\left(\left\|\phi^{1}\right\|+\left\|\phi^{2}\right\|\right)\left\|\phi_{i}^{1}-\phi_{i}^{2}\right\| \leq C \varepsilon^{\frac{N-2}{2}}\left\|\phi_{i}^{1}-\phi_{i}^{2}\right\| .
\end{gathered}
$$

Similarly

$$
\begin{aligned}
& || U_{i}+\left|\phi_{i}^{1}\right|+\left.\left.\left|\phi_{i}^{2}\right|\right|^{\frac{p-3}{2}}\left|U_{j}+\right| \phi_{j}^{1}|+| \phi_{j}^{2}||^{\frac{p-1}{2}}|| \phi_{j}^{1}|+| \phi_{j}^{2}||\left|\phi_{i}^{1}-\phi_{i}^{2}\right|\right|_{\frac{2 N}{N+2}} \\
& \quad \leq\left|U_{i}+\right| \phi_{i}^{1}|+| \phi_{i}^{2}||_{2^{*}}^{\frac{p-3}{2}}\left|U_{j}+\right| \phi_{j}^{1}|+| \phi_{j}^{2}||_{2^{*}}^{\frac{p-1}{2}}|| \phi_{j}^{1}|+| \phi_{j}^{2}||_{2^{*}}\left|\phi_{i}^{1}-\phi_{i}^{2}\right|_{2^{*}} \\
& \quad \leq C \varepsilon^{\frac{N-2}{2}}\left\|\phi_{i}^{1}-\phi_{i}^{2}\right\|,
\end{aligned}
$$

and estimating the remaining terms on the right hand side in (D.5) in the very same way (the terms are obtained one from the other after a permutation of the exponents), we deduce that

$$
\left|\tilde{Q}_{\mathbf{d}, \boldsymbol{\tau}, \varepsilon}^{i}\left(\phi^{1}\right)-\tilde{Q}_{\mathbf{d}, \boldsymbol{\tau}, \varepsilon}^{i}\left(\phi^{2}\right)\right|_{\frac{2 N}{N+2}} \leq C \varepsilon^{\frac{N-2}{2}}\left\|\phi^{1}-\phi^{2}\right\|
$$

for any $\phi^{1}, \phi^{2} \in Y_{\varepsilon}$, as desired. 


\section{APPENDix E.}

In this appendix we prove the validity of estimate (3.29).

In this proof we write $U_{i}$ for $U_{\delta_{i}, \xi_{i}}, \psi_{i}^{h}$ for $\psi_{\delta_{i}, \xi_{i}}^{h}$, and $P$ for $P_{\varepsilon}$ in order to keep the notation as short as possible.

Step 1) We consider $\partial_{s_{k, h}} L_{\mathbf{d}, \boldsymbol{\tau}, \varepsilon}^{i}(\phi)$, with $\phi \in \mathbf{K}_{\mathbf{d}, \boldsymbol{\tau}, \varepsilon}^{\perp}$. Let us focus at first on the case $k=i$. We have

$$
\begin{aligned}
\partial_{s_{i, h}} & L_{\mathbf{d}, \boldsymbol{\tau}, \varepsilon}^{i}(\phi)=\Pi_{i}^{\perp} \circ i^{*}\left[p(p-1)\left(P U_{i}\right)^{p-2}\left(P \psi_{i}^{h}\right) \phi_{i}+\right. \\
& +\frac{(p-1)(p-3)}{4} \sum_{j \neq i} \beta_{i j}\left(\mu_{j}^{-\frac{1}{p-1}} P U_{j}\right)^{\frac{p+1}{2}}\left(\mu_{i}^{-\frac{1}{p-1}} P U_{i}\right)^{\frac{p-5}{2}} \mu_{i}^{-\frac{1}{p-1}}\left(P \psi_{i}^{h}\right) \phi_{i} \\
& \left.+\frac{(p+1)(p-1)}{4} \sum_{j \neq i} \beta_{i j}\left(\mu_{j}^{-\frac{1}{p-1}} P U_{j}\right)^{\frac{p-1}{2}}\left(\mu_{i}^{-\frac{1}{p-1}} P U_{i}\right)^{\frac{p-3}{2}} \mu_{i}^{-\frac{1}{p-1}}\left(P \psi_{i}^{h}\right) \phi_{j}\right] .
\end{aligned}
$$

Therefore, using the continuity of $\Pi^{\perp}$ and $i^{*}$, and the fact that $0 \leq P U_{i} \leq U_{i}$, we deduce that

$$
\begin{aligned}
\left\|\partial_{s_{i, h}} L_{\mathbf{d}, \boldsymbol{\tau}, \varepsilon}^{i}(\phi)\right\| \leq & C\left|U_{i}^{p-2}\left(P \psi_{i}^{h}\right) \phi_{i}\right|_{\frac{2 N}{N+2}}+C \sum_{j \neq i}\left|U_{i}^{\frac{p-3}{2}} U_{j}^{\frac{p-1}{2}}\left(P \psi_{i}^{h}\right) \phi_{j}\right|_{\frac{2 N}{N+2}} \\
& +C(p-3) \sum_{j \neq i}\left|U_{i}^{\frac{p-5}{2}} U_{j}^{\frac{p+1}{2}}\left(P \psi_{i}^{h}\right) \phi_{i}\right|_{\frac{2 N}{N+2}} \\
\leq & C \underbrace{\left|U_{i}^{p-2} \psi_{i}^{h} \phi_{i}\right|_{\frac{2 N}{N+2}}}_{=:(I)}+C \sum_{j \neq i}^{|U_{i}^{\frac{p-3}{2}} \underbrace{\frac{p-1}{2}}_{j} \psi_{i}^{h} \phi_{j}|_{\frac{2 N}{N+2}}} \\
& +C(p-3) \sum_{j \neq i} \underbrace{\left|U_{i}^{\frac{p-5}{2}} U_{j}^{\frac{p+1}{2}} \psi_{i}^{h} \phi_{i}\right|_{\frac{2 N}{N+2}}}_{=:(I I)}+n . t .
\end{aligned}
$$

where n.t stays for "negligible terms", and denotes a quantity which is a small $o$ of the previous ones as $\varepsilon \rightarrow 0$. The exact shape of these terms can be computed using Corollary A.2 (similarly as we have already done in Appendix B), but it's not so important for what follows and hence it is omitted for the sake of brevity. Notice also that term $(I I I)$ is present only in case $N=3$, in which case $p=5$.

Now, by the Hölder and the Sobolev inequalities, and using Lemmas A.6-A.9, we compute for $h=0$

$$
\begin{aligned}
|(I)| & \leq C\left|U_{i}^{p-2} \psi_{i}^{h}\right|_{\frac{N}{2}}\left\|\phi_{i}\right\| \leq C \delta_{i}^{-1}\left(\int_{\Omega_{\varepsilon}} U_{i}^{2^{*}}\right)^{\frac{2}{N}}\left\|\phi_{i}\right\| \leq C \delta_{i}^{-1}\left\|\phi_{i}\right\|, \\
|(I I)| & \leq C\left|U_{i}^{\frac{p-3}{2}} U_{j}^{\frac{p-1}{2}} \psi_{i}^{h}\right|_{\frac{N}{2}}\left\|\phi_{j}\right\| \leq C \delta_{i}^{-1}\left(\int_{\Omega_{\varepsilon}} U_{i}^{\frac{N}{N-2}} U_{j}^{\frac{N}{N-2}}\right)^{\frac{2}{N}}\left\|\phi_{j}\right\| \\
& \leq C \delta_{j}\left(\left|\log \delta_{i}\right|+\left|\log \delta_{j}\right|\right)^{\frac{2}{N}}\left\|\phi_{j}\right\|,
\end{aligned}
$$

and in case $N=3$

$$
\begin{aligned}
|(I I I)| & \leq C\left\|\phi_{i}\right\|\left|U_{j}^{3} \psi_{i}^{h}\right|_{\frac{3}{2}} \leq C \delta_{i}^{-1}\left\|\phi_{i}\right\|\left(\int_{\Omega_{\varepsilon}} U_{i}^{\frac{3}{2}} U_{j}^{\frac{9}{2}}\right)^{\frac{2}{3}} \\
& \leq C \delta_{i}^{-1}\left(\delta_{i} \delta_{j}\right)^{\frac{1}{2}}\left\|\phi_{i}\right\| .
\end{aligned}
$$


Moreover, for $h=1, \ldots, N$,

$$
\begin{aligned}
|(I)| \leq C\left|U_{i}^{p-2} \psi_{i}^{h}\right|_{\frac{N}{2}}\left\|\phi_{i}\right\| \leq C \delta_{i}^{-1}\left(\int_{\Omega_{\varepsilon}} U_{i}^{\frac{3 N}{N-2}}\left|x_{h}-\xi_{i, h}\right|^{\frac{N}{2}}\right)^{\frac{2}{N}}\left\|\phi_{i}\right\| \leq C \delta_{i}^{-1}\left\|\phi_{i}\right\|, \\
|(I I)| \leq C\left\|\phi_{j}\right\|\left|U_{i}^{\frac{p-3}{2}} U_{j}^{\frac{p-1}{2}} \psi_{i}^{h}\right|_{\frac{N}{2}} \leq C \delta_{i}^{-1}\left\|\phi_{j}\right\|\left(\int_{\Omega_{\varepsilon}} U_{i}^{\frac{2 N}{N-2}} U_{j}^{\frac{N}{N-2}}\left|x_{h}-\xi_{i, h}\right|^{\frac{N}{2}}\right)^{\frac{2}{N}} \\
\quad \leq C \delta_{i}^{-1}\left\|\phi_{j}\right\|\left(\delta_{i} \delta_{j}\right),
\end{aligned}
$$

and for $N=3$

$$
|(I I I)| \leq C\left\|\phi_{i}\right\|\left|U_{j}^{3} \psi_{i}^{h}\right|_{\frac{3}{2}} \leq C \delta_{i}^{-1}\left\|\phi_{i}\right\|\left(\int_{\Omega_{\varepsilon}} U_{j}^{\frac{9}{2}} U_{i}^{\frac{9}{2}}\left|x_{h}-\xi_{i, h}\right|^{\frac{3}{2}}\right)^{\frac{2}{3}} \leq C \delta_{i}^{\frac{1}{2}} \delta_{j}^{\frac{1}{2}}\left\|\phi_{i}\right\| .
$$

Altogether, recalling the ansatz (2.5) and that $\|\phi\| \leq C \varepsilon^{\frac{N-2}{2}}$ by Lemma 3.3, we conclude that for every $i=1, \ldots, m$ and $h=0, \ldots, N$

$$
\left\|\partial_{s_{i, h}} L_{\mathbf{d}, \boldsymbol{\tau}, \varepsilon}^{i}(\phi)\right\|_{H_{0}^{1}\left(\Omega_{\varepsilon}\right)} \leq C \varepsilon^{\frac{N-3}{2}}
$$

as desired.

Now, if $k \neq i$, we have still to consider

$$
\begin{aligned}
\partial_{s_{k, h}} & L_{\mathbf{d}, \boldsymbol{\tau}, \varepsilon}^{i}(\phi) \\
& =\Pi_{i}^{\perp} \circ i^{*}\left[\frac{(p-1)(p+1)}{4} \beta_{k i}\left(\mu_{k}^{-\frac{1}{p-1}} P U_{k}\right)^{\frac{p-1}{2}}\left(\mu_{i}^{-\frac{1}{p-1}} P U_{i}\right)^{\frac{p-3}{2}} \mu_{k}^{-\frac{1}{p-1}} P \psi_{k}^{h} \phi_{i}\right. \\
& \left.+\frac{(p-1)(p+1)}{4} \beta_{k i}\left(\mu_{k}^{-\frac{1}{p-1}} P U_{k}\right)^{\frac{p-3}{2}}\left(\mu_{i}^{-\frac{1}{p-1}} P U_{i}\right)^{\frac{p-1}{2}} \mu_{k}^{-\frac{1}{p-1}} P \psi_{k}^{h} \phi_{k}\right] .
\end{aligned}
$$

This term can be treated exactly as the one with $k=i$ (actually the computations are easier).

Step 2) We consider $\partial_{s_{k, h}} R_{\mathbf{d}, \boldsymbol{\tau}, \varepsilon}^{i}(\boldsymbol{\phi})$, and again we present the details only for the (a bit harder) case $k=i$. We have

$$
\begin{aligned}
& \partial_{s_{i, h}} R_{\mathbf{d}, \boldsymbol{\tau}, \varepsilon}^{i}=\Pi_{i}^{\perp} \circ i^{*}\left[\mu_{i}^{-\frac{1}{p-1}} p\left(\left(P U_{i}\right)^{p-1} P \psi_{i}^{h}-U_{i}^{p-1} \psi_{i}^{h}\right)\right. \\
&\left.\quad+\frac{p-1}{2} \sum_{j \neq i} \beta_{i j}\left(\mu_{j}^{-\frac{1}{p-1}} P U_{j}\right)^{\frac{p+1}{2}}\left(\mu_{i}^{-\frac{1}{p-1}} P U_{i}\right)^{\frac{p-3}{2}}\left(\mu_{i}^{-\frac{1}{p-1}} P \psi_{i}^{h}\right)\right]
\end{aligned}
$$

and hence

$$
\begin{aligned}
& \left\|\partial_{s_{i, h}} R_{\mathbf{d}, \boldsymbol{\tau}, \varepsilon}^{i}\right\| \leq C\left|\left(P U_{i}\right)^{p-1} P \psi_{i}^{h}-U_{i}^{p-1} \psi_{i}^{h}\right|_{\frac{2 N}{N+2}}+C \sum_{j \neq i}\left|U_{j}^{\frac{p+1}{2}} U_{i}^{\frac{p-3}{2}} P \psi_{i}^{h}\right|_{\frac{2 N}{N+2}} \\
& \leq C\left|P U_{i}^{p-1}\right| P \psi_{i}^{h}-\psi_{i}^{h} \|_{\frac{2 N}{N+2}}+C|| P U_{i}^{p-1}-U_{i}^{p-1}\left|\psi_{i}^{h}\right|_{\frac{2 N}{N+2}}+C \sum_{j \neq i}\left|U_{j}^{\frac{p+1}{2}} U_{i}^{\frac{p-3}{2}} \psi_{i}^{h}\right|_{\frac{2 N}{N+2}}+\text { n.t. } \\
& \leq C \underbrace{\left|U_{i}^{p-1}\right| P \psi_{i}^{h}-\psi_{i}^{h} \|_{\frac{2 N}{N+2}}}_{=:(I)}+C \underbrace{\left|\psi_{i}^{h} U_{i}^{p-2}\right| P U_{i}-\left.U_{i}\right|_{\frac{2 N}{N+2}}}_{=:(I I)}+C \sum_{j \neq i} \underbrace{\left|U_{j}^{\frac{p+1}{2}} U_{i}^{\frac{p-3}{2}} \psi_{i}^{h}\right|_{\frac{2 N}{N+2}}}_{=:(I I I)}+\text { n.t. }
\end{aligned}
$$

where we used Lemma A.4 and the fact that $0 \leq P U_{i} \leq U_{i}$. 
As in the first step, using the lemmas collected in Appendix A, we estimate separately the three terms on the right hand side, starting from the case $h=0$ :

$$
|(I)| \leq C\left|U_{i}^{\frac{4}{N-2}}\left(\delta_{i}^{\frac{N-4}{2}}+\frac{\delta_{i}^{\frac{3}{2} N-4}}{\left|x-a_{i}\right|^{N-2}}\right)\right|_{\frac{2 N}{N+2}}
$$

$$
\begin{aligned}
& \leq C \delta_{i}^{\frac{N-4}{2}}\left(\int_{\Omega_{\varepsilon}} U_{i}^{\frac{8 N}{(N+2)(N-2)}}\right)^{\frac{N+2}{2 N}}+C \delta_{i}^{\frac{3}{2} N-4}\left(\int_{\Omega_{\varepsilon}} \frac{U_{i}^{\frac{8 N}{(N+2)(N-2)}}}{\left|x-a_{i}\right|^{\frac{2 N(N-2)}{N+2}}}\right)^{\frac{N+2}{2 N}} \\
& \leq C \delta_{i}^{\frac{N-4}{2}+\frac{N-2}{2}}+C \delta_{i}^{\frac{3}{2} N-4-\frac{N-2}{2}} \leq C \delta_{i}^{N-3}, \\
|(I I)| \leq & C \delta_{i}^{-1}\left|U_{i}^{p-1}\left(\delta_{i}^{\frac{N-2}{2}}+\frac{\delta_{i}^{\frac{3 N}{2}-3}}{\left|x-a_{i}\right|^{N-2}}\right)\right|_{\frac{2 N}{N+2}}
\end{aligned}
$$

$$
\leq C \delta_{i}^{\frac{N-4}{2}}\left(\int_{\Omega_{\varepsilon}} U_{i}^{\frac{8 N}{(N+2)(N-2)}}\right)^{\frac{N+2}{2 N}}+C \delta_{i}^{\frac{3}{2} N-4}\left(\int_{\Omega_{\varepsilon}} \frac{U_{i}^{\frac{8 N}{(N+2)(N-2)}}}{\left|x-a_{i}\right|^{\frac{2 N(N-2)}{N+2}}}\right)^{\frac{N+2}{2 N}} \leq C \delta_{i}^{N-3}
$$

$$
|(I I I)| \leq C \delta_{i}^{-1}\left|U_{j}^{\frac{p+1}{2}} U_{i}^{\frac{p-1}{2}}\right|_{\frac{2 N}{N+2}} \leq C \delta_{i}^{-1}\left(\delta_{i}^{\frac{2 N}{N+2}} \delta_{j}^{\frac{2 N}{N+2}}\right)^{\frac{N+2}{2 N}} \leq C \delta_{j} .
$$

Analogously, for $h=1, \ldots, N$, we have

$$
\begin{aligned}
|(I)| \leq C \mid U_{i}^{\frac{4}{N-2}} & \left.\left(\delta_{i}^{\frac{N-2}{2}}+\frac{\delta_{i}^{\frac{3}{2} N-4}}{\left|x-a_{i}\right|^{N-2}}\right)\right|_{\frac{2 N}{N+2}} \leq C \delta_{i}^{\frac{N-2}{2}+\frac{N-2}{2}}+C \delta_{i}^{\frac{3}{2} N-4-\frac{N-2}{2}} \leq C \delta_{i}^{N-3} \\
|(I I)| & \leq C \delta_{i}^{-1}\left|U_{i}^{\frac{6}{N-2}}\right| x_{h}-\xi_{i, h}\left|\left(\delta_{i}^{\frac{N-2}{2}}+\frac{\delta_{i}^{\frac{3}{2} N-3}}{\left|x-a_{i}\right|^{N-2}}\right)\right|_{\frac{2 N}{N+2}} \\
& \leq C \delta_{i}^{\frac{N-4}{2}}\left(\int_{\Omega_{\varepsilon}} U_{i}^{\frac{12 N}{(N+2)(N-2)}}\left|x_{h}-\xi_{i, h}\right|^{\frac{2 N}{N+2}}\right)^{\frac{N+2}{2 N}} \\
& +C \delta_{i}^{\frac{3}{2} N-4}\left(\int_{\Omega_{\varepsilon}} U_{i}^{\frac{12 N}{(N+2)(N-2)}} \frac{\left|x_{h}-\xi_{i, h}\right|^{\frac{2 N}{N+2}}}{\left|x-a_{i}\right|^{\frac{2 N(N-2)}{N+2}}}\right)^{\frac{N+2}{2 N}} \\
& \leq C \delta_{i}^{\frac{N-4}{2}+\frac{N-2}{2}}+C \delta_{i}^{\frac{3}{2} N-4+\frac{2-N}{2}} \leq C \delta_{i}^{N-3}
\end{aligned}
$$

$$
\begin{aligned}
|(I I I)| & \leq C \delta_{i}^{-1}\left|U_{j}^{\frac{N}{N-2}} U_{i}^{\frac{4}{N-2}}\right| x_{h}-\left.\xi_{i, h}\right|_{\frac{2 N}{N+2}} \\
& \leq C \delta_{i}^{-1}\left(\int_{\Omega_{\varepsilon}} U_{i}^{\frac{8 N}{(N+2)(N-2)}} U_{j}^{\frac{2 N^{2}}{(N+2)(N-2)}}\left|x_{h}-\xi_{i, h}\right|^{\frac{2 N}{N+2}}\right)^{\frac{N+2}{2 N}} \\
& \leq C \delta_{i}^{-1}\left(\delta_{i}^{\frac{4 N}{N+2}} \delta_{j}^{\frac{N^{2}}{N+2}}+\delta_{i}^{\frac{4 N}{N+2}} \int_{\Omega_{\varepsilon}} U_{j}^{\frac{2 N^{2}}{(N+2)(N-2)}}+\delta_{j}^{\frac{N^{2}}{N+2}} \int_{\Omega_{\varepsilon}} U_{i}^{\frac{8 N}{(N+2)(N-2)}}\left|x_{h}-\xi_{i, h}\right|^{\frac{2 N}{N+2}}\right)^{\frac{N+2}{2 N}} \\
& \leq C \delta_{i}^{-1}\left(\delta_{i}^{2} \delta_{j}+\delta_{i}^{2} \delta_{j}^{\frac{N}{2}}\left|\log \delta_{j}\right|^{\frac{N+2}{2 N}}\right)=o\left(\varepsilon^{\frac{N-3}{2}}\right) .
\end{aligned}
$$

Notice that the logarithm appears in the computations only for $N=4$, see Lemma A.7. 
Collecting together the previous estimates, we deduce that $\left\|\partial_{s_{i, h}} R_{\mathbf{d}, \boldsymbol{\tau}, \varepsilon}^{i}\right\| \leq C \varepsilon^{\frac{N-3}{2}}$, as desired.

Step 3) We consider $\partial_{s_{k, h}} N_{\mathbf{d}, \boldsymbol{\tau}, \varepsilon}^{i}(\phi)$, with $\phi \in \mathbf{K}_{\mathbf{d}, \boldsymbol{\tau}, \varepsilon}^{\perp}$. Once again, we focus on the case $k=i$, which presents the heaviest calculations. We have

$$
\begin{aligned}
\partial_{s_{k, h}} N_{\mathbf{d}, \boldsymbol{\tau}, \varepsilon}^{i}(\phi) & \\
& =\Pi_{i}^{\perp} \circ i^{*}\left[\left(f^{\prime}\left(\mu_{i}^{-\frac{1}{p-1}} P U_{i}+\phi_{i}\right)-f^{\prime}\left(\mu_{i}^{-\frac{1}{p-1}} P U_{i}\right)-f^{\prime \prime}\left(\mu_{i}^{-\frac{1}{p-1}} P U_{i}\right) \phi_{i}\right) \mu_{i}^{\frac{p-2}{p-1}} P \psi_{i}^{h}\right. \\
& +\frac{p-1}{2} \sum_{j \neq i} \beta_{i j}\left|\mu_{j}^{-\frac{1}{p-1}} P U_{j}+\phi_{j}\right|^{\frac{p+1}{2}}\left|\mu_{i}^{-\frac{1}{p-1}} P U_{i}+\phi_{i}\right|^{\frac{p-3}{2}} \mu_{i}^{-\frac{1}{p-1}} P \psi_{i}^{h} \\
& -\frac{p-1}{2} \sum_{j \neq i} \beta_{i j}\left(\mu_{j}^{-\frac{1}{p-1}} P U_{j}\right)^{\frac{p+1}{2}}\left(\mu_{i}^{-\frac{1}{p-1}} P U_{i}\right)^{\frac{p-3}{2}} \mu_{i}^{-\frac{1}{p-1}} P \psi_{i}^{h} \\
& -\frac{(p-1)(p-3)}{4} \sum_{j \neq i} \beta_{i j}\left(\mu_{j}^{-\frac{1}{p-1}} P U_{j}\right)^{\frac{p+1}{2}}\left(\mu_{i}^{-\frac{1}{p-1}} P U_{i}\right)^{\frac{p-5}{2}} \mu_{i}^{-\frac{1}{p-1}} P \psi_{i}^{h} \phi_{i} \\
& \left.-\frac{(p+1)(p-1)}{4} \sum_{j \neq i} \beta_{i j}\left(\mu_{j}^{-\frac{1}{p-1}} P U_{j}\right)^{\frac{p-1}{2}}\left(\mu_{i}^{-\frac{1}{p-1}} P U_{i}\right)^{\frac{p-3}{2}} \mu_{i}^{-\frac{1}{p-1}} P \psi_{i}^{h} \phi_{j}\right]
\end{aligned}
$$

Therefore, if $N=3$ we can use the Lagrange theorem to deduce that

$$
\begin{aligned}
& \left\|\partial_{s_{k, h}} N_{\mathbf{d}, \boldsymbol{\tau}, \varepsilon}^{i}(\phi)\right\| \leq C\left|\left(U_{i}^{2}+\phi_{i}^{2}\right) \phi_{i}^{2} P \psi_{i}^{h}\right|_{\frac{6}{5}} \\
& +C \sum_{j \neq i}\left(\left\|U_{i}+\left|\phi_{i}\right||| U_{j}+\left|\phi _ { j } \left\|\left.^{2} \phi_{j} P \psi_{i}^{h}\right|_{\frac{6}{5}}+|| U_{j}+\left|\phi_{j} \| \phi_{i} P \psi_{i}^{h}\right|_{\frac{6}{5}}\right.\right.\right.\right. \\
& \left.+\left|U_{j}^{3} \phi_{i} P \psi_{i}^{h}\right|_{\frac{6}{5}}+\left|U_{i} U_{j}^{2} \phi_{j} P \psi_{i}^{h}\right|_{\frac{6}{5}}\right)
\end{aligned}
$$

if on the other hand $N=4$, then by direct computations

$$
\begin{aligned}
& \left\|\partial_{s_{k, h}} N_{\mathbf{d}, \boldsymbol{\tau}, \varepsilon}^{i}(\phi)\right\| \leq C\left|\phi_{i}^{2} P \psi_{i}^{h}\right|_{\frac{4}{3}} \\
& \quad+C \sum_{j \neq i}\left(\left|U_{j} \phi_{j} P \psi_{i}^{h}\right|_{\frac{4}{3}}+\left|\phi_{j}^{2} P \psi_{i}^{h}\right|_{\frac{4}{3}}+\left|U_{j} \phi_{j} P \psi_{i}^{h}\right|_{\frac{4}{3}}\right) .
\end{aligned}
$$

In both cases, using the lemmas in Appendix A as in the previous steps, it is not difficult to check that

$$
\left\|\partial_{s_{k, h}} N_{\mathbf{d}, \boldsymbol{\tau}, \varepsilon}^{i}(\phi)\right\| \leq C \varepsilon^{\frac{N-3}{2}}
$$

This completes the proof of (3.29).

\section{REFERENCES}

[1] N. Akhmediev and A. Ankiewicz. Partially coherent solitons on a finite background. Phys. Rev. Lett., 82:2661, 1999.

[2] A. Bahri and J.-M. Coron. On a nonlinear elliptic equation involving the critical Sobolev exponent: the effect of the topology of the domain. Comm. Pure Appl. Math., 41(3):253-294, 1988.

[3] T. Bartsch. Bifurcation in a multicomponent system of nonlinear Schrödinger equations. J. Fixed Point Theory Appl.. 13: 37-50, 2013. 19(3):200-207, 2006.

[4] G. Bianchi and H. Egnell. A note on the sobolev inequality. Journal of Functional Analysis, 100(1):18 - 24, 1991.

[5] H. Brézis and L. Nirenberg. Positive solutions of nonlinear elliptic equations involving critical Sobolev exponents. Comm. Pure Appl. Math., 36(4):437-477, 1983. 
[6] J. Byeon, Y. Sato, and Z.-Q. Wang. Pattern formation via mixed attractive and repulsive interactions for nonlinear Schrödinger systems. J. Math. Pures Appl. (9), 106(3):477-511, 2016.

[7] L. A. Caffarelli, B. Gidas, and J. Spruck. Asymptotic symmetry and local behavior of semilinear elliptic equations with critical sobolev growth. Communications on Pure and Applied Mathematics, 42(3):271-297, 1989.

[8] Z. Chen and C.-S. Lin. Asymptotic behavior of least energy solutions for a critical elliptic system. Int. Math. Res. Not. IMRN, (21):11045-11082, 2015.

[9] Z. Chen and W. Zou. Positive least energy solutions and phase separation for coupled Schrödinger equations with critical exponent. Arch. Ration. Mech. Anal., 205(2):515-551, 2012.

[10] Z. Chen and W. Zou. Positive least energy solutions and phase separation for coupled Schrödinger equations with critical exponent: higher dimensional case. Calc. Var. Partial Differential Equations, 52(1-2):423-467, 2015.

[11] M. Clapp, M. Musso, and A. Pistoia. Multipeak solutions to the Bahri-Coron problem in domains with a shrinking hole. J. Funct. Anal., 256(2):275-306, 2009.

[12] M. Clapp and T. Weth. Two solutions of the Bahri-Coron problem in punctured domains via the fixed point transfer. Commun. Contemp. Math., 10(1):81-101, 2008.

[13] J.-M. Coron. Topologie et cas limite des injections de Sobolev. C. R. Acad. Sci. Paris Sér. I Math., 299(7):209$212,1984$.

[14] Y. Ge, M. Musso, and A. Pistoia. Sign changing tower of bubbles for an elliptic problem at the critical exponent in pierced non-symmetric domains. Communications in Partial Differential Equations, 35(8):1419-1457, 2010.

[15] Y. Guo, B. Li, and J. Wei. Entire nonradial solutions for non-cooperative coupled elliptic system with critical exponents in $\mathbb{R}^{3}$. J. Differential Equations, 256(10):3463-3495, 2014.

[16] J. L. Kazdan and F. W. Warner. Remarks on some quasilinear elliptic equations. Comm. Pure Appl. Math., 28(5):567-597, 1975.

[17] A. Iacopetti and G. Vaira. Sign-changing blowing-up solutions for the Brezis-Nirenberg problem in dimensions four and five. Preprint arXiv:1504.05010, 2015.

[18] G. Li, S. Yan, and J. Yang. An elliptic problem with critical growth in domains with shrinking holes. $J$. Differential Equations, 198(2):275-300, 2004.

[19] M. Musso and A. Pistoia. Erratum to: Persistence of Coron's solutions in nearly critical problems. Ann. Scuola Norm. Sup. Pisa Cl. Sci (5), 8, 207-209, 2009.

[20] M. Musso and A. Pistoia. Persistence of Coron's solutions in nearly critical problems. Ann. Scuola Norm. Sup. Pisa Cl. Sci (5), 6, 331-357, 2007.

[21] M. Musso and A. Pistoia. Sign changing solutions to a Bahri-Coron's problem in pierced domains. Discrete Contin. Dyn. Syst., 21(1):295-306, 2008.

[22] A. Pistoia. The Ljapunov-Schmidt reduction for some critical problems. In Concentration Analysis and Applications to PDE - ICTS Workshop, Bangalore, January 2012, Trends in Mathematics, pages 69-83. Springer, Basel, 2013.

[23] A. Pistoia and H. Tavares. Spiked solutions for Schrödinger systems with Sobolev critical expnent: the cases of competitive and weakly cooperative interactions. Preprint arXiv:1605.03776, 2016, to appear in J. Fixed Point Theory Appl..

[24] O. Rey. Sur un problème variationnel non compact: l'effet de petits trous dans le domaine. C. R. Acad. Sci. Paris Sér. I Math., 308(12):349-352, 1989.

[25] Y. Sato and Z.-Q. Wang. Least energy solutions for nonlinear Schrödinger systems with mixed attractive and repulsive couplings. Adv. Nonlinear Stud., 15(1):1-22, 2015.

[26] Y. Sato and Z.-Q. Wang. Multiple positive solutions for Schrödinger systems with mixed couplings. Calc. Var. Partial Differential Equations, 54(2):1373-1392, 2015.

[27] B. Sirakov. Least energy solitary waves for a system of nonlinear Schrödinger equations in $\mathbb{R}^{n}$. Comm. Math. Phys., 271(1):199-221, 2007.

[28] N. Soave. On existence and phase separation of solitary waves for nonlinear Schrödinger systems modelling simultaneous cooperation and competition. Calc. Var. Partial Differential Equations, 53(3-4):689-718, 2015.

[29] N. Soave and H. Tavares. New existence and symmetry results for least energy positive solutions of Schrödinger systems with mixed competition and cooperation terms. J. Differential Equations, 261(1):505-537, 2016.

[30] E. Timmermans. Phase separation of Bose-Einstein condensates. Phys. Rev. Lett., 81:5718-5721, Dec 1998. 
Angela Pistoia

Dipartimento di Metodi e Modelli Matematici, Università di Roma "La Sapienza", Via Antonio Scarpa 16, 00161 Roma (Italy),

E-mail address: angela.pistoia@uniroma1.it

Nicola SoAve

Mathematisches Institut, Justus-Liebig-Universität Giessen,

Arndtstrasse 2, 35392 Giessen (Germany),

E-mail address: nicola.soave@gmail.com, Nicola.Soave@math.uni-giessen.de 